\title{
Eigenfunction Statistics on Quantum Graphs
}

\author{
S. Gnutzmann ${ }^{1}$, J.P. Keating ${ }^{2}$, F. Piotet ${ }^{2,3}$ \\ ${ }^{1}$ School of Mathematical Sciences, University of Nottingham, Nottingham, NG7 2RD, United Kingdom \\ ${ }^{2}$ School of Mathematics, University of Bristol, Bristol, BS8 1TW, United Kingdom \\ ${ }^{3}$ Department of Physics of Complex Systems, The Weizmann Institute of Science, 76100 Rehovot, Israel
}

May 6, 2010

\begin{abstract}
We investigate the spatial statistics of the energy eigenfunctions on large quantum graphs. It has previously been conjectured that these should be described by a Gaussian Random Wave Model, by analogy with quantum chaotic systems, for which such a model was proposed by Berry in 1977. The autocorrelation functions we calculate for an individual quantum graph exhibit a universal component, which completely determines a Gaussian Random Wave Model, and a system-dependent deviation. This deviation depends on the graph only through its underlying classical dynamics. Classical criteria for quantum universality to be met asymptotically in the large graph limit (i.e. for the non-universal deviation to vanish) are then extracted. We use an exact field theoretic expression in terms of a variant of a supersymmetric $\sigma$ model. A saddle-point analysis of this expression leads to the estimates. In particular, intensity correlations are used to discuss the possible equidistribution of the energy eigenfunctions in the large graph limit. When equidistribution is asymptotically realized, our theory predicts a rate of convergence that is a significant refinement of previous estimates. The universal and system-dependent components of intensity correlation functions are recovered by means of an exact trace formula which we analyse in the diagonal approximation, drawing in this way a parallel between the field theory and semiclassics. Our results provide the first instance where an asymptotic Gaussian Random Wave Model has been established microscopically for eigenfunctions in a system with no disorder.
\end{abstract}

Keywords : Quantum ergodicity, Random Wave Model, Criteria for universality, Rate of universality, Trace formulae, Nonlinear supersymmetric $\sigma$ model.

\section{Introduction}

Gaussian Random Wave Models are commonly used to describe the statistical properties of the energy eigenfunctions of chaotic quantum systems. The original idea was introduced in 1977 by Berry [13], who proposed that a random function $\psi$ with Gaussian distribution

$$
\mathcal{N}(\psi) \propto e^{-\frac{\beta}{2} \int \psi^{*}\left(\boldsymbol{r}_{1}\right) c^{-1}\left(\boldsymbol{r}_{1}, \boldsymbol{r}_{2} \mid e_{n}\right) \psi\left(\boldsymbol{r}_{2}\right) d \boldsymbol{r}_{1} d \boldsymbol{r}_{2}}
$$

could, in the semiclassical limit, reproduce all the spatial autocorrelation functions

$$
C\left(\left\{\boldsymbol{x}_{i}\right\}_{i \in \mathbb{N}_{q}} ;\left\{\boldsymbol{y}_{j}\right\}_{j \in \mathbb{N}_{p}}\right) \equiv \frac{1}{|S|} \int_{S} \prod_{i=1}^{q} \psi_{n}^{*}\left(\boldsymbol{x}_{\boldsymbol{i}}+\boldsymbol{q}\right) \prod_{j=1}^{p} \psi_{n}\left(\boldsymbol{y}_{j}+\boldsymbol{q}\right) d \boldsymbol{q},
$$


of a chaotic eigenfunction $\psi_{n}$ of energy $e_{n}$. Here, $S$ is a small volume that shrinks in the semiclassical limit but does so slowly enough to contain an increasing number of oscillations of $\psi_{n}$, and $\beta$ in (1) is 1 if time-reversal symmetry is conserved, in which case $\psi$ is chosen real, and 2 if this symmetry is broken, in which case $\psi$ is complex. From a semiclassical calculation of $C\left(\boldsymbol{r}_{1}, \boldsymbol{r}_{2}\right)$, Berry deduced that the covariance $c\left(\boldsymbol{r}_{1}, \boldsymbol{r}_{2}\right)$ in (11) is the free quantum propagator from $\boldsymbol{r}_{\mathbf{2}}$ to $\boldsymbol{r}_{\mathbf{1}}$.

This is one of the central conjectures in the field of quantum chaos. Essentially, it asserts that the local statistics of quantum chaotic eigenfunctions correspond, in the semiclassical limit, to those of random superpositions of plane waves, and so are universal.

Following Berry, the universal Gaussian Random Wave Model has been refined to incorporate systems-specific features. For example, in quantum billiards, it does not fulfill the necessary boundary conditions. In this case, Hortikar and Srednicki [29] suggested replacing the covariance with the semiclassical approximation [14, 27] to the propagator of the system. This Gaussian model satisfies the boundary conditions and has the property that the direct path contribution to the semiclassical formula corresponds to Berry's conjecture. Further understandings and refinements of this systemdependent Gaussian Random Wave model are given in [42, 43, 44], for example.

It is important to emphasize that to-date effort has mainly been directed towards deriving the consequences of the Random Wave Model and its refinements, assuming its validity. Numerical tests strongly support the predictive value of the Random Wave Model. However, in no system has its validity yet been established or derived microscopically.

We tackle here the problem of the validity of the Gaussian Random Wave Model on quantum graphs (a variant of this model was introduced in [26]). Quantum graphs are favorable systems to gain some insights on the mechanisms responsible for random waves models to hold because, depending on their topology and their boundary conditions, their behaviors range from chaotic [34, 35], where a random model is expected to hold, to intermediate [8, 7, 31, 10, 30], where such models should fail. Without any prior assumption on the nature of the quantum graph, one can evaluate its autocorrelation functions

$$
C\left(\left\{\boldsymbol{x}_{\boldsymbol{i}}\right\}_{i \in \mathbb{N}_{q}} ;\left\{\boldsymbol{y}_{\boldsymbol{j}}\right\}_{j \in \mathbb{N}_{p}}\right) \equiv \lim _{N \rightarrow \infty} \frac{1}{N} \sum_{n=1}^{N} \prod_{i=1}^{q} \psi_{n}^{*}\left(\boldsymbol{x}_{i}\right) \prod_{j=1}^{p} \psi_{n}\left(\boldsymbol{y}_{j}\right),
$$

where $\left\{\boldsymbol{x}_{\boldsymbol{i}}\right\}_{i \in \mathbb{N}_{q}}$ and $\left\{\boldsymbol{y}_{j}\right\}_{j \in \mathbb{N}_{p}}$ are points on the graph. In fact, we focus on the autocorrelations for $q=p=1$, and for $q=p$ with $\left\{\boldsymbol{x}_{i}\right\}_{i \in \mathbb{N}_{q}}=\left\{\boldsymbol{y}_{j}\right\}_{j \in \mathbb{N}_{q}}$. The other autocorrelations are believed to vanish due to additional complex phases that fluctuate strongly. The result obtained for $q=p=1$ is exact and yields a universal covariance $c$ which defines the unique candidate for the Gaussian model on quantum graphs. It should be emphasized that this does not contradict the construction of Gaussian Random Wave Models with a system-dependent correction in analogy to Urbina and Richter's guess for billiards [42]. Indeed, our autocorrelation functions are defined by averaging over the whole energy spectrum. Such an average on Urbina and Richter's random functions also kills the system-dependent correction and leads to a covariance given by the free propagator, namely, to Berry's universal model. The system dependency found in the 
autocorrelation functions of higher degree evaluated here is of different nature. It is not a refinement of a universal Random Wave Model, but it rather measures how chaotic a given quantum graph is from the energy eigenfunctions perspective. Interestingly, this non-universal term is found to depend on the quantum graph only through its classical dynamics. This provides us a way to estimate the deviation from quantum universality in terms of a classical quantity, and so to discuss criteria for the Random Wave Model to hold in the large-graph limit, and cases where it fails, such as Neumann star graphs [11]. Our results provide the first instance where an asymptotic Gaussian Random Wave Model has been established microscopically for eigenfunctions in a system with no disorder.

It would be a major achievement to show that our result for the autocorrelation functions (3) in the case of quantum graphs also applies to other quantum systems. If this is the case, the deviations from universality vanish in the semiclassical limit in chaotic billiards, which explains why such deviations have indeed never been found, whereas they must prevail over the universal part in non-chaotic systems. For chaotic systems, the corrections would reveal the rate of approach to universality as $\hbar \rightarrow 0$. Finally, if such a formula was found, its ability to describe systems with mixed phase spaces could be studied and compared with the empirical results [2, 3] and alternative approaches based on bifurcation theory and singularity-dominated strong fluctuations [32].

The moments and autocorrelations of second degree (i.e. intensity correlations) play a particularly important role in quantum chaos, because they suffice to measure the spreading of the energy eigenfunctions, and they can be rigorously controlled. According to [40], the high energy eigenfunctions of a classically ergodic system should become uniformly spread over the surface of constant energy, a property known as quantum ergodicity. This claim has found rigorous proofs in [17], [16] and [50] for example, where the authors consider compact manifolds with ergodic geodesic flows, quantized ergodic maps and ergodic billiards respectively. The main tool used in these works is an Egorov estimate, which, in the case of quantum maps, reads

$$
\left\|U_{M}^{\dagger k} O p(f) U_{M}^{k}-O p\left(f \circ M^{k}\right)\right\| \leq \mathrm{const} \cdot \hbar
$$

for $M$ a map, $f$ any smooth function on the configuration space, and $U$ and $O p(f)$ their quantized analogs. A version of (4) also holds for continuous Hamiltonian systems. However, the Egorov method does not provide any information on the rate with which quantum ergodicity is reached. This much harder problem is investigated in [21, 47, 48, 49, 18, 1, 39].

In fact, quantum ergodicity is significantly more difficult to tackle on quantum graphs than on other chaotic systems. The reason is the non-existence of a deterministic classical map, and hence, of an Egorov estimate. In [9], quantum ergodicity is proved for graphs related to quantum maps by using the Egorov property on the underlying quantum maps. On the other hand, it is shown in [10, 11, 30] that some graphs, namely star graphs, are not quantum ergodic. Here, our result for the autocorrelation functions (3) with $p=2$ enables us to expound a criterion for graphs to become quantum ergodic. A summary of our results in this special case has already been given in [24]. Moreover, our method also yields the rate of quantum ergodicity in terms of 
the classical dynamics, when quantum ergodicity does occur. The result obtained is a significant refinement of the previous estimates in [18].

The reader not interested in the derivation of the formulae can directly jump to Section 7 where the final formulae are given and exploited. The rest of the text is structured as follows. In the sections 2 and 3 quantum graphs are defined, and the autocorrelation functions together with other statistical quantities of interest are introduced. In particular, a first type of trace formulae is developed in 3.4 and 3.5 An exact field theoretic expression for the autocorrelation functions is developed in Section 4, and a second type of trace formulae is presented in 4.2. Then, two different contributions to the exact expression for the autocorrelation functions are extracted and calculated in sections 5 and 6 Section 7 compares these two contributions and illustrates them with a few examples. Section 8 discusses our results and gives an outlook on possible implications.

\section{Quantum Graphs}

\subsection{Definitions}

A metric graph $G$ is a set of $V \in \mathbb{N}$ points, called the vertices, and of $B \in \mathbb{N}$ bonds of positive lengths $L=\left(L_{1}, \cdots, L_{B}\right)$ linking some pairs of vertices. The topology of a graph is determined by its connectivity matrix $C$, namely the $V \times V$ matrix

$$
\left.C_{i, j}=C_{j, i}=\# \text { bonds connecting the vertices } i \text { and } j\right\} \text {. }
$$

If $C_{i, i}=0$ and $C_{i, j} \leq 1$ for all $i, j \in \mathbb{N}_{V}$, the graph is said to be simple. The valency $v_{i}$ of a vertex $i \in \mathbb{N}_{V}$ is defined by $v_{i}=\sum_{j=1}^{V} C_{i, j}$. The valencies are always all supposed positive. A point on a graph is specified by a pair $\left(b, x_{b}\right)$, where $b \in \mathbb{N}_{B}$ determines the bond and $x_{b} \in\left[0, L_{b}\right]$ determines the position of this point on $b$.

Each bond of a metric graph can be traversed in two possible directions, denoted by $d \in\{+,-\}$. A pair $\beta=(b, d)$ then denotes a directed bond, and $\hat{\beta}=(b,-d)$ stands for its reverse partner. The vertex from which a directed bond $\beta$ emerges is written $o \beta$ and the vertex to which it leads is written $t \beta$. In particular, $o \beta=t \hat{\beta}$ is always fulfilled. We suppose the set of directed bonds to be ordered so that, by abuse of language, any directed bond $\beta$ can also be seen as an element of $\mathbb{N}_{2 B}$.

A quantum graph is a metric graph $G$ that is turned into a quantum system. In order to do this, the $\mathbb{C}$-linear space

$$
\mathcal{H}=\left\{\Psi=\bigoplus_{b=1}^{B} \psi_{b} \mid \psi_{b}, \psi_{b}^{\prime}, \psi_{b}^{\prime \prime} \in L^{2}\left(\left[0, L_{b}\right]\right)\right\}
$$

is introduced, and its elements are referred to as wave functions. This space is endowed with the scalar product defined by

$$
(\Psi, \Phi) \equiv \sum_{b=1}^{B} \int_{0}^{L_{b}} \psi_{b}^{*}(x) \phi_{b}(x) d x
$$


for any $\Psi, \Phi \in \mathcal{H}$. The number $\psi_{b}\left(x_{b}\right)$ is interpreted as the value of the wave function $\Psi$ at the point $\left(b, x_{b}\right)$ of $G$. One can define an operator $H$ acting on $\mathcal{H}$ as

$$
H \bigoplus_{b=1}^{B} \psi_{b}=\bigoplus_{b=1}^{B}-\psi_{b}^{\prime \prime} .
$$

This is the expression of the free quantum particle Hamiltonian on each bond. The restriction of $H$ on the subset $\mathcal{H}_{0} \subset \mathcal{H}$ of wave functions vanishing at the vertices is symmetric. A wave function $\Psi \in \mathcal{H}_{0}$ is called a Dirichlet wave function. A Schrödinger operator on a metric graph (and thus a quantum graph) can be defined as a self-adjoint extension of $H$. However, we will follow a slightly different definition using the scattering approach [34]. We first give a brief overview of this approach and then discuss its relation to self-adjoint extensions of $H$.

For any real number $k>0$, the solutions of the equation $H \Psi=k^{2} \Psi$ form the subspace

$$
\tilde{\mathcal{A}}(k)=\left\{\bigoplus_{b=1}^{B} \sum_{d=+,-} a_{b d} \tilde{e}_{b d}(k) \mid a_{\beta} \in \mathbb{C}, \forall \beta=(b, d) \in \mathbb{N}_{2 B}\right\},
$$

where, for $b \in \mathbb{N}_{B}$ and $d \in\{+,-\}$,

$$
\tilde{e}_{b d}(k)=e^{i d k\left(x-\frac{L_{b}}{2}\right)} .
$$

A wave function in $\tilde{\mathcal{A}}(k)$ is then characterized by $2 B$ waves of wave number $k$, each of which carries a complex amplitude $a_{\beta}$ corresponding to its value at the mid-point of the bond.

Let us introduce $2 B$ formal symbols $\left|e_{\beta}\right\rangle, \beta \in \mathbb{N}_{2 B}$, and the set $\mathcal{A}$ of their possible linear combinations over $\mathbb{C}$. The set $\mathcal{A}$ is a $2 B$-dimensional $\mathbb{C}$-linear space called amplitude space, and it is endowed with the hermitian scalar product defined by

$$
\left\langle e_{\beta^{\prime}} \mid e_{\beta}\right\rangle=\delta_{\beta, \beta^{\prime}} .
$$

It can be seen as the direct product $\mathcal{A}=\mathcal{A}_{b} \otimes \mathcal{A}_{d}$ of a $B$-dimensional bond space $\mathcal{A}_{b}$ and a two-dimensional direction space $\mathcal{A}_{d}$. For each $k>0$, there is a natural one-to-one mapping

$$
\Psi=\bigoplus_{b=1}^{B} \sum_{d=+,-} a_{b d} \tilde{e}_{b d}(k) \mapsto|\boldsymbol{a}\rangle=\sum_{\beta=1}^{2 B} a_{\beta}\left|e_{\beta}\right\rangle
$$

between $\tilde{\mathcal{A}}(k)$ and $\mathcal{A}$. If $\Psi_{1} \mapsto\left|\boldsymbol{a}_{\mathbf{1}}\right\rangle$ and $\Psi_{2} \mapsto\left|\boldsymbol{a}_{\mathbf{2}}\right\rangle$ by this mapping, the scalar products in the spaces $\tilde{\mathcal{A}}(k)$ and $\mathcal{A}$ translate

$$
\left(\Psi_{1}, \Psi_{2}\right)=\left\langle\boldsymbol{a}_{\mathbf{1}}\left|L+\frac{\sin (k L)}{k} \sigma_{1}^{d}\right| \boldsymbol{a}_{2}\right\rangle,
$$

where $\sigma_{1}^{d}$ stands for the first Pauli matrix acting on $\mathcal{A}_{d}$, and $L$ denotes the $2 B \times 2 B$ diagonal matrix

$$
L_{\beta^{\prime} \beta}=\delta_{\beta, \beta^{\prime}} L_{\beta}
$$


Here and henceforth, the length of a directed bond is the length of the bond on which it is supported. In particular, $L_{\beta}=L_{\hat{\beta}}$ is always fulfilled. The identity (13) shows that the mapping (12) does not preserve length and orthogonality in general.

In the scattering approach to quantum graphs the values at each vertex $i \in \mathbb{N}_{V}$ of the $v_{i}$ waves emerging from this vertex and of the $v_{i}$ waves incoming to this vertex are related through some fixed matrix $\sigma^{i}$. If $\left|\boldsymbol{a}_{\text {out }}^{i}\right\rangle$ and $\left|\boldsymbol{a}_{\text {in }}^{i}\right\rangle$ denote the $v_{i}$-dimensional vectors containing the values at vertex $i$ of the emerging waves and of the incoming waves respectively, this relation reads

$$
\left|\boldsymbol{a}_{\mathrm{out}}^{i}\right\rangle=\sigma^{i}\left|\boldsymbol{a}_{\mathrm{in}}^{i}\right\rangle .
$$

A wave function $\Psi \in \tilde{\mathcal{A}}(k)$ conserves the probability current if and only if the $V$ matrices $\sigma^{i}$ are all unitary. The components of the $V$ outgoing and incoming vectors $\left|\boldsymbol{a}_{\text {out }}^{i}\right\rangle$ and $\left|\boldsymbol{a}_{\text {in }}^{i}\right\rangle$ can then be grouped together to form the $2 B$-dimensional vectors $\left|\boldsymbol{a}_{\text {out }}\right\rangle$ and $\left|\boldsymbol{a}_{\text {in }}\right\rangle$ respectively. These vectors are related to $|\boldsymbol{a}\rangle$ in (12) through

$$
\left|\boldsymbol{a}_{\text {out }}\right\rangle=T^{\dagger}(k)|\boldsymbol{a}\rangle \quad \text { and } \quad\left|\boldsymbol{a}_{\text {in }}\right\rangle=T(k)|\boldsymbol{a}\rangle
$$

where $T(k)$ is the $2 B \times 2 B$ diagonal matrix $T(k)=e^{i k \frac{L}{2}}$. This matrix contains the phases gained by the $2 B$ waves of wave number $k$ when they travel along half the bonds on which they are supported. It is referred to as the propagation matrix. Moreover, the $V$ identities (15) become

$$
\left|\boldsymbol{a}_{\text {out }}\right\rangle=S\left|\boldsymbol{a}_{\text {in }}\right\rangle,
$$

where $S$ is the $2 B \times 2 B$ unitary matrix, called scattering matrix, defined by

$$
S_{\beta^{\prime} \beta}= \begin{cases}\sigma_{\beta^{\prime} \beta}^{i} & \text { if } o \beta^{\prime}=t \beta=i \\ 0 & \text { otherwise }\end{cases}
$$

Putting (16) and (17) together yields

$$
U(k)|\boldsymbol{a}\rangle=|\boldsymbol{a}\rangle, \quad \text { with } \quad U(k)=T(k) S T(k) .
$$

The $2 B \times 2 B$ matrix $U(k)$ is called the quantum map or evolution map of the graph. It is unitary since both $T(k)$ and $S$ are unitary.

Equation (19) shows that imposing the conservation of probability current through fixed unitary matrices $\sigma^{i}$ restricts the possible amplitudes $|\boldsymbol{a}\rangle$ and the possible wave numbers $k>0$. Indeed, the secular equation

$$
\operatorname{det}(1-U(k))=0
$$

must be satisfied for (19) to admit non-trivial solutions. This equation is satisfied for a sequence

$$
0 \leq k_{1}<k_{2}<\ldots<k_{v}<k_{v+1}<\ldots \rightarrow \infty
$$

called the spectrum of the quantum graph, and the square of these wave numbers are the quantized energies. If the bond lengths $L_{1}, \ldots, L_{B}$ are independent over $\mathbb{Q}$, there is typically a normalized vector $\left|\boldsymbol{a}^{v}\right\rangle$ in $\mathcal{A}$ for any $v \in \mathbb{N}$ that satisfies $U\left(k_{v}\right)\left|\boldsymbol{a}^{v}\right\rangle=\left|\boldsymbol{a}^{v}\right\rangle$ and so that any other vector satisfying this equation is of the form $z\left|\boldsymbol{a}^{v}\right\rangle$ for some $z \in \mathbb{C}$. The 
vector $\left|\boldsymbol{a}^{v}\right\rangle$ then provides the amplitudes of the eigenfunction $\Psi^{v}$ satisfying $H \Psi^{v}=k_{v}^{2} \Psi^{v}$ by the mapping (12). Incommensurability of the bond lengths and this non-degeneracy property will be assumed henceforth.

It is well-known [25] that the mean number of allowed wave numbers in $[0, K]$ is $N(K) \equiv K \bar{d}$, where the mean level density $\bar{d}$ reads

$$
\bar{d} \equiv \frac{\operatorname{tr} L}{2 \pi} .
$$

For any $k>0$, the unitarity of $U(k)$ ensures the existence of an orthonormal basis $\{|n, k\rangle\}_{n \in \mathbb{N}_{2 B}}$ of $\mathbb{C}^{2 B}$ and of $2 B$ real numbers $\left\{\phi_{n}(k)\right\}_{n \in \mathbb{N}_{2 B}}$ such that

$$
U(k)|n, k\rangle=e^{i \phi_{n}(k)}|n, k\rangle
$$

These sets can be ordered by imposing the inequalities

$$
-2 \pi<\phi_{2 B}(0) \leq \phi_{2 B-1}(0) \leq \ldots \leq \phi_{2}(0) \leq \phi_{1}(0) \leq 0
$$

and by requiring the $2 B$ eigencurves $k \mapsto \phi_{n}(k)$ to be $C^{\infty}$. This smoothness condition can indeed be realized since the map $U(k)$ depends on $k$ in an analytic way. Taking a derivative with respect to $k$ on both sides of 23) leads to

$$
\phi_{n}^{\prime}(k)=\langle n, k|L| n, k\rangle \in\left[L_{\min }, L_{\max }\right],
$$

where $L_{\min }$ and $L_{\max }$ denote the minimal and maximal bond lengths on the graph.

A quantum graph is time-reversal invariant if its quantum map satisfies $\operatorname{tr}\left(U(k)^{\mathcal{T}}\right)^{n}=$ $\operatorname{tr} U(k)^{n}$ for all $k \geq 0$ and integers $n$. Here and henceforth, the generalized transposition $A^{\mathcal{T}}$ of a linear transformation $A$ is defined by

$$
A^{\mathcal{T}}=\sigma_{1}^{d} A^{T} \sigma_{1}^{d}
$$

$A^{T}$ being the transpose of $A$. It satisfies $A^{\mathcal{T}^{\mathcal{T}}}=A$. Since $T(k)^{\mathcal{T}}=T(k)$, a graph is time-reversal invariant if and only if its scattering matrix satisfies $\operatorname{tr}\left(S^{\mathcal{T}}\right)^{n}=\operatorname{tr} S^{n}$ for all integers $n$. Obviously, $S^{\mathcal{T}}=S$ implies time-reversal invariance. Note, however that replacing

$$
S \mapsto S^{\prime}=e^{-i \theta} S e^{i \theta} \quad|\boldsymbol{a}\rangle \mapsto\left|\boldsymbol{a}^{\prime}\right\rangle=e^{-i \theta}|\boldsymbol{a}\rangle
$$

where $\theta=\operatorname{diag}\left(\theta_{1}, \ldots, \theta_{2 B}\right)$ is a diagonal real matrix is equivalent to choosing a different reference phase for the amplitudes. We will call such a transformation a (passive) gauge transformation - it neither affects the spectrum nor the condition described above for time-reversal invariance. The latter can now be reformulated: a quantum graph is time-reversal invariant if and only if there is a (possibly trivial) gauge transformation $S \mapsto S^{\prime}=e^{-i \theta} S e^{i \theta}$ such that $S^{\prime \mathcal{T}}=S^{\prime}$. For time-reversal invariant graphs we will henceforth assume that the reference phases have been chosen such that $S^{\mathcal{T}}=S$ holds. There remains a residual gauge freedom to which we will return later when we discuss the wave function statistics in quantum graphs.

The set of all time-reversal invariant graphs form the orthogonal symmetry class, and the set of all quantum graphs violating this property form the unitary symmetry 
class. We will frequently use the parameter $\kappa$ which takes the values

$$
\kappa= \begin{cases}1 & \text { in the unitary class, and } \\ 2 & \text { in the orthogonal class. }\end{cases}
$$

Note that the parameter $\kappa$ that we use here is linked to the parameter $\beta$ used in randommatrix theory to distinguish symmetry classes by $\kappa=2 / \beta$.

We have already mentioned that the scattering approach described above is not the only way to define quantum graphs. The other frequently used definition is based on self-adjoint extensions of $H$ in $(8)$ defined on the Dirichlet domain $\mathcal{H}_{0}$ (see [15] and references therein). A complete description of all possible self-adjoint extensions was given in [33]. In general each self-adjoint extension is equivalent to energy-dependent matrices $\sigma^{\mathrm{KS}, i}(k)$ relating the outgoing amplitudes to the incoming amplitudes of $\Psi \in$ $\tilde{\mathcal{A}}(k)$ at each vertex $i$ instead of $(15)$. These matrices can then be grouped together to form a global unitary scattering matrix $S^{\mathrm{KS}}(k)$ as in (18), and a global quantum map $U^{\mathrm{KS}}(k)=T(k) S^{\mathrm{KS}}(k) T(k)$ satisfying the secular equation (20). The two definitions of quantum graphs have a certain overlap as there is a subset of self-adjoint extensions which leads to energy-independent scattering matrices. It is shown in [6] and [15] that any scattering matrix $S^{\mathrm{KS}}$ defining a self-adjoint operator $H$ admits a limit $S_{\infty}^{\mathrm{KS}}$ as $k$ tends to infinity, and moreover, it is argued in [6] that a scattering matrix $S^{\mathrm{KS}}$ and its limit $S_{\infty}^{\mathrm{KS}}$ share the same spectral statistics. The coincidence of these statistics comes from the fact that they are properties at asymptotically large wave number $k$. Hence, one can deduce that the eigenfunction statistics of $S^{\mathrm{KS}}$ and $S_{\infty}^{\mathrm{KS}}$ also coincide. As a consequence the eigenfunction statistics of quantum graphs defined following the self-adjoint extension approach can be recovered from the eigenfunction statistics of quantum graphs defined through the scattering approach by substituting $S_{\infty}^{\mathrm{KS}}$ for $S^{\mathrm{KS}}$.

Henceforth, the scattering matrix $S$ always refers to the matrix in (18) obtained from the scattering approach. It can be any $2 B \times 2 B$ unitary matrix such that $S_{\beta^{\prime} \beta}$ vanishes if $t \beta \neq o \beta^{\prime}$. A possible choice is the so-called Neumann scattering matrix, which is defined at each vertex $i \in \mathbb{N}_{V}$ by

$$
\sigma_{\beta^{\prime} \beta}^{i}=\frac{2}{v_{i}}-\delta_{\beta, \beta^{\prime}}, \quad \forall t \beta=o \beta^{\prime}=i .
$$

Quantum graphs with this choice of scattering matrix at each vertex will be called Neumann quantum graphs.

In general, a quantum graph is then specified by a pair $(G, S)$ where $G$ is a metric graph and $S$ is a scattering matrix on $G$. The class of possible scattering matrices $S$ on $G$ contains all the asymptotic matrices $S_{\infty}^{\mathrm{KS}}$ obtained from the self-adjoint extension approach. There are however some scattering matrices that are acceptable from the scattering point of view but not from the second approach. An example is given by the Direct Fourier Transform (DFT) graphs [25], for which the scattering processes at vertex $i \in \mathbb{N}_{V}$ are described by the $v_{i} \times v_{i}$ unitary matrix

$$
\sigma_{\beta^{\prime} \beta}^{i}=\frac{1}{\sqrt{v_{i}}} e^{2 \pi i \frac{n^{i}(\beta) n^{i}\left(\beta^{\prime}\right)}{v_{i}}}, \quad \forall t \beta=o \beta^{\prime}=i,
$$

where $n^{i}$ is a surjective assignment of an integer in $\mathbb{N}_{v_{i}}$ to each directed bond around $i$ such that $n^{i}(\hat{\beta})=n^{i}(\beta)$. With these matching conditions, the wave functions $\left\{\Psi^{v}\right\}_{v \in \mathbb{N}}$ 
obtained from the amplitudes $\left\{\left|\boldsymbol{a}^{v}\right\rangle\right\}_{v \in \mathbb{N}}$ and the spectrum $\left\{k_{v}\right\}_{v \in \mathbb{N}}$ by (12) are in general not orthogonal to each other in $\mathcal{H}$, which shows that $H$ acting on the wave functions satisfying (30) is not self-adjoint. By contrast, the Neumann scattering matrices (29) do lead to a self-adjoint Laplace operator.

Both examples of scattering matrices were defined in terms of symmetric unitary matrices at each vertex $\sigma^{i}=\sigma^{i \mathcal{T}}$. As a consequence $S^{\mathcal{T}}=S$ and the a quantum graph obeys time-reversal symmetry. One may break time-reversal symmetry by adding a magnetic field to the graph. In the scattering approach adding a magnetic field which is constant on every bond is straightforward. Let $A$ be the diagonal matrix that contains the magnetic field strengths. It obeys $A_{\beta}=-A_{\hat{\beta}}$. The corresponding quantum map is

$$
U(k)=e^{i(k+A) L / 2} S e^{i(k+A) L / 2} \equiv T(k) S_{A} T(k)
$$

and the magnetic field effectively just changes the scattering matrix $S \mapsto S_{A}=e^{i A L / 2} S e^{i A L / 2}$. If $S=S^{\mathcal{T}}$ and the graph is multiply connected (that is, it contains cycles) then the magnetic field generally breaks the time reversal invariance.

Henceforth, the metric graphs $G$ considered are assumed simple. The reason for this assumption is to simplify some notations and calculations. However, if a graph contains a directed bond $\beta$ such that $o \beta=t \beta$, a Neumann vertex can be added on the bond $b$ supporting $\beta$ to destroy the loop $b$ without modifying the quantum dynamics. Similarly, if the graph has two directed bonds $\beta, \beta^{\prime}$ such that $o \beta=o \beta^{\prime}$ and $t \beta=t \beta^{\prime}$, a Neumann vertex can be added on the bond $b$ supporting $\beta$ to destroy this parallel connection without modifying the dynamics. Hence, any graph can be made simple by adding sufficiently many Neumann vertices, and this process does not change the quantum dynamics. One can thus assume the graph simple without loss of generality.

\subsection{Classical Dynamics}

With any quantum graph, one can associate a bistochastic classical map $M$ defined by

$$
M_{\beta \beta^{\prime}} \equiv\left|U_{\beta \beta^{\prime}}(k)\right|^{2}=\left|S_{\beta \beta^{\prime}}\right|^{2},
$$

where $U(k)$ is the quantum map and $S$ is the scattering matrix. The matrix $M$ describes a Markov process on the graph, which is the classical counterpart of the quantum dynamics defined by $S$. The uniform vector

$$
|1\rangle \equiv \frac{1}{\sqrt{2 B}} \sum_{\beta=1}^{2 B}\left|e_{\beta}\right\rangle
$$

is an eigenvector of $M$ of eigenvalue 1 , and its hermitian conjugate $\langle 1|$ is a left eigenvectors of $M$ of eigenvalue 1 . Besides, the Perron-Frobenius theorem [28] ensures that the spectrum of $M$ lies on or within the complex unit disc.

A graph is said to be ergodic if and only if, for any $\beta, \beta^{\prime} \in \mathbb{N}_{2 B}$, there is a discrete time $n \in \mathbb{N}$ for which the transition probability $\left\langle e_{\beta^{\prime}}\left|M^{n}\right| e_{\beta}\right\rangle$ is positive. This condition is equivalent to the non-degeneracy of the eigenvalue 1 of $M$. Any non-ergodic graph $(G, S)$ is the union of several ergodic components, that is $(G, S)=\bigcup_{i=1}^{k}\left(G_{i}, S_{i}\right)$ for 
some integer $k>1$. The eigenvalue 1 has degeneracy $k$, and the $k$ vectors that are uniform on one component $\left(G_{i}, S_{i}\right)$ and zero on the others form a basis of this eigenspace.

Let us write $M_{\epsilon}=e^{-2 \epsilon} M$ for an ergodic classical map $M$ and for some $\epsilon>0$. The sum of all classical paths from $\beta \in \mathbb{N}_{2 B}$ to $\beta^{\prime} \in \mathbb{N}_{2 B}$ followed with $M_{\epsilon}$ can be written

$$
\left(\frac{M_{\epsilon}}{1-M_{\epsilon}}\right)_{\beta^{\prime} \beta}=\left(M_{\epsilon}+M_{\epsilon}^{2}+M_{\epsilon}^{3}+\ldots\right)_{\beta^{\prime} \beta} .
$$

It becomes singular as $\epsilon$ approaches zero due to the eigenvalue 1 of $M$. Let $M=$ $D_{M}+N_{M}$ be the Jordan decomposition of $M$ into a diagonalizable part $D_{M}$ and a nilpotent part $N_{M}$ commuting with each other. Let $\left\{\lambda_{j}\right\}_{j \in \mathbb{N}_{2 B}}$ be the $2 B$ eigenvalues of $D_{M}$, and let $\{|j\rangle\}_{j \in \mathbb{N}_{2 B}}$ be corresponding normalized eigenvectors in $\mathcal{A}$ with $|1\rangle$ as in (33). Then, it is straight forward to check that $\langle 1 \mid j\rangle=\delta_{1, j}$. This fact enables one to extract the singular part of 34 and write

$$
\frac{M_{\epsilon}}{1-M_{\epsilon}}=\frac{e^{-2 \epsilon}}{1-e^{-2 \epsilon}}|1\rangle\langle 1|+R_{\epsilon},
$$

where the remainder $R_{\epsilon}$ is such that $R \equiv \lim _{\epsilon \rightarrow 0} R_{\epsilon}$ exists and satisfies $\langle 1| R=0$ and $R|1\rangle=0$. The first and second terms in the right-hand side of 35 will respectively be referred to as uniform and massive components. If $m_{i}=1-\lambda_{i}$ for $i=2, \ldots, 2 B$ denote the $2 B-1$ non-zero eigenvalues of $1-M$, the massive component satisfies

$$
\operatorname{tr} R=\sum_{i=2}^{2 B} \frac{1-m_{i}}{m_{i}}
$$

The eigenvalues $\left\{m_{i}\right\}_{i \in \mathbb{N}_{2 B}}$ of $1-M$ are called masses. They all lie in the closed disc of radius 1 and centered at 1 in the complex plane, and the zero mass $m_{1}=0$ is nondegenerate.

\section{Eigenfunction Statistics}

\subsection{Wave function correlation functions}

Let $(G, S)$ be a quantum graph, $\left\{k_{v}\right\}$ be its spectrum, and $\left\{\boldsymbol{a}^{v}\right\} \subset \mathbb{C}^{2 B}$ be a set of normalized amplitude vectors defining the eigenfunctions $\left\{\Psi^{v}\right\}$ as in (12). Let us consider $2 B$ complex random variables $a_{\beta}$ and investigate the existence of a joint probability density function $\varphi(\boldsymbol{a})=\varphi\left(a_{1}, \ldots, a_{2 B}\right)$ satisfying

$$
\begin{aligned}
\left\langle\prod_{k=0}^{p-1} a_{\beta_{k}}^{*} \prod_{l=0}^{q-1} a_{\beta_{l}^{\prime}}\right\rangle & \equiv \lim _{K \rightarrow \infty} \frac{1}{N(K)} \sum_{k_{\nu} \leq K} \frac{\operatorname{tr} L}{2 B\langle L\rangle_{\nu}} \prod_{k=0}^{p-1} a_{\beta_{k}}^{\nu *} \prod_{l=0}^{q-1} a_{\beta_{l}^{\prime}}^{v} \\
& =\int_{\mathbb{C}^{2 B}} \prod_{k=0}^{p-1} a_{\beta_{k}}^{*} \prod_{l=0}^{q-1} a_{\beta_{l}^{\prime}} \varphi(\boldsymbol{a}) d \boldsymbol{a}^{*} d \boldsymbol{a},
\end{aligned}
$$

for any choice of $\beta_{0}, \ldots, \beta_{p-1}, \beta_{0}^{\prime}, \ldots, \beta_{q-1}^{\prime} \in \mathbb{N}_{2 B}$ with $p, q \in \mathbb{N}_{0}$. Here, the measure $d \boldsymbol{a}^{*} d \boldsymbol{a}$ denotes the product of the $2 B$ flat Lebesgue measures $d a_{\beta}^{*} d a_{\beta}$ in the complex plane, and the notation $\langle O\rangle_{v}$ for a $2 B \times 2 B$ matrix $O$ stands for $\langle O\rangle_{v}=\left\langle\boldsymbol{a}^{v}|O| \boldsymbol{a}^{v}\right\rangle$. 
The first line in (37) defines the wave function correlation functions. The peculiar factor $\frac{\operatorname{tr} L}{2 B\langle L\rangle_{v}}$ in this definition is introduced for further calculational convenience. For large graphs with extended wave functions this factor is expected to be close to unity. Indeed, it has generally a tiny effect on the wave function statistics. It will be seen later that with the inclusion of this factor 37 does not depend on the particular values of the incommensurate bond lengths. Moreover, performing an average over the spectrum of the quantum graph in presence of this factor, such as in 37), amounts to averaging the same quantity over all the eigenfunctions $|n, k\rangle$ of $U(k)$ and then integrating over all $k \in(0, \infty)$. Indeed, it is proven in [12] that graphs with incommensurate bond lengths obey

$$
\lim _{K \rightarrow \infty} \frac{1}{N(K)} \sum_{k_{v} \leq K} \frac{\operatorname{tr} L}{2 B\langle L\rangle_{v}}\langle O\rangle_{v}^{q}=\lim _{K \rightarrow \infty} \frac{1}{K} \int_{0}^{K} \frac{1}{2 B} \sum_{n=1}^{2 B}\langle n, k|O| n, k\rangle^{q} d k .
$$

for any $2 B \times 2 B$ matrix $O$ and any non-negative integer $q$.

The identity (39) shows that the joint probability density function $\varphi(\boldsymbol{a})$ in (38) is normalized. Indeed, choosing $q=0$ in this formula leads to

$$
\langle 1\rangle \equiv \lim _{K \rightarrow \infty} \frac{1}{N(K)} \sum_{k_{v} \leq K} \frac{\operatorname{tr} L}{2 B\langle L\rangle_{v}}=\lim _{K \rightarrow \infty} \frac{1}{K} \int_{0}^{K} \frac{1}{2 B} \sum_{n=1}^{2 B} 1=1 .
$$

Moreover, it also provides an exact expression for the covariance of $\varphi(\boldsymbol{a})$. Indeed, the equality (39) with $q=1$ and $O=\left|e_{\beta^{\prime}}\right\rangle\left\langle e_{\beta}\right|$ yields

$$
\begin{aligned}
\left\langle a_{\beta^{\prime}}^{*} a_{\beta}\right\rangle & \equiv \lim _{K \rightarrow \infty} \frac{1}{N(K)} \sum_{k_{\nu} \leq K} \frac{\operatorname{tr} L}{2 B\langle L\rangle_{v}}\left\langle v \mid e_{\beta^{\prime}}\right\rangle\left\langle e_{\beta} \mid v\right\rangle \\
& =\lim _{K \rightarrow \infty} \frac{1}{K} \int_{0}^{K} \frac{1}{2 B} \sum_{n=1}^{2 B}\left\langle n, k \mid e_{\beta^{\prime}}\right\rangle\left\langle e_{\beta} \mid n, k\right\rangle d k=\frac{\delta_{\beta, \beta^{\prime}}}{2 B},
\end{aligned}
$$

by orthonormality of the families $\{|n, k\rangle\}_{n \in \mathbb{N}_{2 B}}$. This derivation of the covariance relies on the incommensurability of bond lengths. We will show later in Subsection 3.4 that the restriction to incommensurable bond lengths can be lifted.

Further properties of the joint probability density can be derived considering its invariance under gauge transformation of the form described in (27). This discussion has to treat systems with and without time-reversal invariance separately and we will start with the unitary class (broken time-reversal invariance). In this class we are free to choose a gauge and one expects that all correlation functions which are not gauge invariant will vanish. This implies that the non-trivial correlation functions 37 have the same number of complex conjugated amplitudes as non-conjugated amplitudes (that is $p=q)$. It is thus sufficient to consider the autocorrelation functions

$$
C_{[\alpha]} \equiv\left\langle\left|a_{\alpha_{0}}\right|^{2} \ldots\left|a_{\alpha_{q-1}}\right|^{2}\right\rangle .
$$

where $[\alpha] \equiv\left[\alpha_{0}, \ldots, \alpha_{q-1}\right]$ is a vector containing $q \in \mathbb{N}_{0}$ directed bonds $\alpha_{j} \in \mathbb{N}_{2 B}$. The integer $q$ is called degree of $C_{[\alpha]}$.

Of particular interest to us are the moments

$$
M_{\alpha, q} \equiv C_{[q \times \alpha]}=\left\langle\left|a_{\alpha}\right|^{2 q}\right\rangle
$$


and the first non-trivial autocorrelation functions

$$
C_{\alpha \alpha^{\prime}} \equiv C_{\left[\alpha \alpha^{\prime}\right]}=\left\langle\left|a_{\alpha}\right|^{2}\left|a_{\alpha^{\prime}}\right|^{2}\right\rangle
$$

which form the symmetric intensity correlation matrix $C_{\alpha \alpha^{\prime}}=C_{\alpha^{\prime} \alpha}$.

For time-reversal invariant systems the property $U(k)^{\mathcal{T}}=U(k)$ of the quantum map implies that one may always choose the phase of its eigenvectors $|n, k\rangle=\sum_{\beta} a_{n, \beta}(k)\left|e_{\beta}\right\rangle$ such that $a_{n, \beta}(k)=a_{n, \hat{\beta}}(k)^{*}$. An equivalent statement is that the wave function on the graph can be chosen real. This has strong implications on wave function statistics - for instance the autocorrelation functions $C_{[\alpha]}$ defined in (42) are invariant under replacing any directed bond in $\alpha=\left(\alpha_{0} \ldots, \alpha_{q-1}\right)$ by its reverse partner $\alpha_{i} \mapsto \hat{\alpha}_{i}$. The joint probability density function then reduces to a product

$$
\varphi(\boldsymbol{a})=\delta^{B}\left(\boldsymbol{a}_{+}-\boldsymbol{a}_{-}^{*}\right) \varphi_{\mathrm{red}}\left(\boldsymbol{a}_{+}\right)
$$

where $\boldsymbol{a}_{+}\left(\boldsymbol{a}_{-}\right)$is the $B$-dimensional vector containing the amplitudes for directed bond $\alpha=(b, d)$ with positive (negative) direction index $d$. For a quantum graph in the orthogonal class it is thus sufficient to consider only the correlation functions in (37) for which all directed bond have a positive direction index. As in the unitary case we also expect for the orthogonal case that correlation functions that depend on a local gauge vanish exactly. Note, that in the orthogonal case not all gauge transformations (27) are allowed. In order to preserve the properties $S^{\mathcal{T}}=S$ and $a_{n, \beta}(k)=a_{n, \hat{\beta}}(k)^{*}$ only gauge transformations with $\theta_{\beta}=-\theta_{\hat{\beta}}$ are allowed. Again the only non-trivial correlation functions are the autocorrelation functions (42).

\subsection{Circular and Gaussian Random Waves Models}

For a large well-connected quantum graph in the unitary symmetry class the quantum map $U(k)$ does generally not have any symmetries. Moreover, in a complex network (e.g. a randomly chosen connected graph) the neighborhood of any bond looks statistically the same. By analogy with the circular ensembles of random matrix theory one is inclined to guess that the joint probability density function $\varphi(\boldsymbol{a})$ for the eigenvectors of the $2 B \times 2 B$ matrix $U(k)$ defined in (38) is invariant under transformations $\boldsymbol{a} \mapsto \boldsymbol{u} \boldsymbol{a}$ for unitary matrices $u$. This implies that the vectors $\boldsymbol{a}$ are uniformly distributed over the unit sphere in $\mathbb{C}^{2 B}$. We will call the guess

$$
\varphi_{\mathrm{CU}}(\boldsymbol{a}) \equiv \frac{(2 B-1) !}{\pi^{2 B}} \delta\left(1-\|\boldsymbol{a}\|^{2}\right)
$$

the Circular Random Wave Model for quantum graphs in the unitary class. The moments and the intensity correlation matrix predicted by the Circular Random Wave Model read

$$
\begin{aligned}
& M_{\mathrm{CU}, \alpha, q}=\frac{q !(2 B-1) !}{(2 B+q-1) !}=\frac{q !}{(2 B)^{q}}\left(1-\frac{q(q-1)}{4 B}+O\left(B^{-2}\right)\right) \\
& C_{\mathrm{CU}, \alpha, \alpha^{\prime}}=\frac{1+\delta_{\alpha \alpha^{\prime}}}{(2 B)(2 B+1)}=\frac{1+\delta_{\alpha \alpha^{\prime}}}{(2 B)^{2}}\left(1-\frac{1}{2 B}+O\left(B^{-2}\right)\right)
\end{aligned}
$$



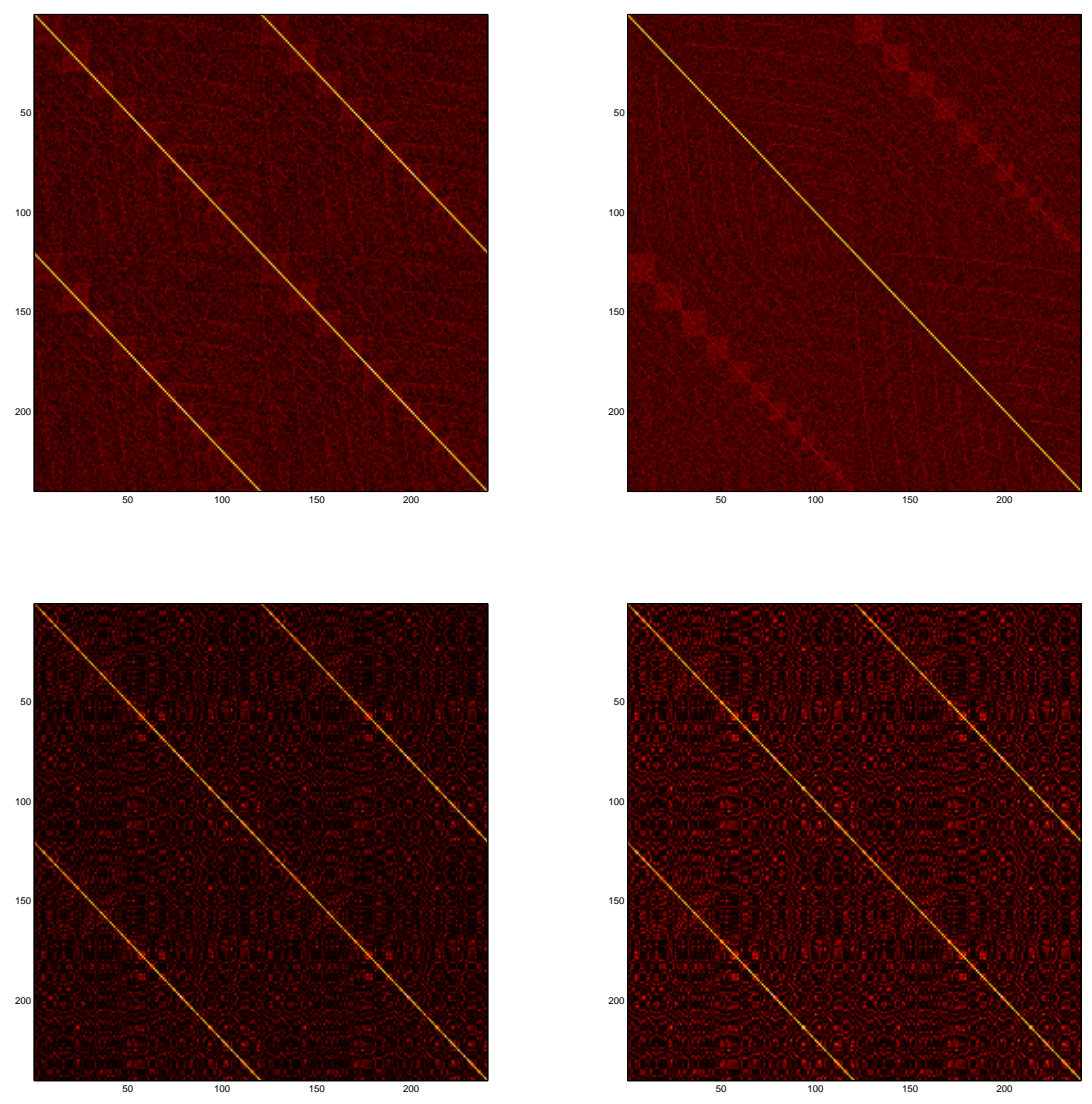

Figure 1: Numerically evaluated intensity correlation matrices $C_{\alpha \alpha^{\prime}}$ defined in 444 for a complete graph with $V=16$ vertices and $B=120$ bonds. In the upper two panels the DFT scattering matrices have been used at each vertex. The lower two panels are for Neumann scattering matrices. For the right two panels time-reversal symmetry has been broken by adding a magnetic field. The directed bonds $\alpha=(b, d)$ have been ordered as $((1,-),(2,-), \ldots,(B,-),(1,+), \ldots,(B,+))$. For the graphs in the orthogonal class on the left side there are four identical blocks as $C_{\alpha \alpha^{\prime}}=C_{\hat{\alpha}, \alpha^{\prime}}=C_{\alpha \hat{\alpha}^{\prime}}=C_{\hat{\alpha} \hat{\alpha}^{\prime}}$. In the unitary case, note that the correlation matrix on the off-diagonal $\alpha=\hat{\alpha}^{\prime}$ remains strongly peaked for Neumann scattering matrices. However the four blocks are no longer the identical (this is not obvious from the picture). For DFT scattering matrices the strong off-diagonal peak almost disappears in the presence of a magnetic field. 
In the limit $B \rightarrow \infty$ (and constant degree $q$ ) one may replace the Circular Random Wave Model by the Gaussian Random Wave Model with the joint probability density

$$
\varphi_{\mathrm{GU}}(\boldsymbol{a}) \equiv \frac{B^{2 B}}{\pi^{2 B}} e^{-2 B\|\boldsymbol{a}\|^{2}} .
$$

The predictions for the moments and the intensity correlation matrix in the Gaussian Random Wave Model read

$$
M_{\mathrm{GU}, \alpha, q}=\frac{q !}{(2 B)^{q}} \quad \text { and } \quad C_{\mathrm{GU}, \alpha, \alpha^{\prime}}=\frac{1+\delta_{\alpha \alpha^{\prime}}}{(2 B)^{2}}
$$

which is equivalent to the leading order of the predictions 47) of the Circular Random Wave Model as $B \rightarrow \infty$.

If the eigenfunction statistics 37) of a family of quantum graphs in the unitary symmetry class are well reproduced by $\varphi_{\mathrm{CU}}(\boldsymbol{a})$ in (46) or by $\varphi_{\mathrm{GU}}(\boldsymbol{a})$ in (48), these formulae provide us with a universal Circular or Gaussian Random Wave Model, which gives access to all the statistical properties of the eigenfunctions. Notice that the exact calculation (41) asserts that (48) is the only possible Gaussian joint probability density function of the type (38), and hence, a non-universal Gaussian model cannot be realized on quantum graphs.

Establishing the possible validity of the Gaussian Random Wave Model (48) would require the calculation of (37) for arbitrary products of amplitudes $a_{\beta}$. Note that the Gaussian Random Wave Model is consistent with the gauge principle, i.e. its prediction for any correlation function that is not explicitly gauge invariant vanishes identically. In what follows we will mainly focus on the explicitly gauge invariant autocorrelation functions (42). However in subsection 4.2 we will show that some low order correlation functions that are not explicitly gauge invariant indeed vanish on the level of the diagonal approximation.

When time-reversal symmetry is conserved one has to take into account that the amplitudes of counter propagating waves on the same bond are complex conjugates, so that the wave function is real. We can thus only expect that a universal joint probability function is invariant under $\boldsymbol{a} \mapsto u \boldsymbol{a}$ where $u$ is a unitary $2 B \times 2 B$ matrix that respects reality of the wave function or, equivalently, that $S \mapsto u^{\dagger} S u$ conserves $S^{\mathcal{T}}=S$. Such unitary matrices have the block structure

$$
u=\left(\begin{array}{ll}
u_{++}^{*} & u_{+-} \\
u_{+-}^{*} & u_{++}
\end{array}\right)
$$

in terms of the direction index $d$. Here $u_{++}$and $u_{+-}$are two $B \times B$ matrices which are only constrained by unitarity of $u$. Unitary matrices with this block structure obey $u^{\mathcal{T}}=u^{\dagger}=u^{-1}$ and are thus in fact orthogonal matrices with respect to $\mathcal{T}$-transposition. The Circular Random Wave Model for the orthogonal class

$$
\varphi_{\mathrm{CO}}(\boldsymbol{a}) \equiv \frac{2^{B-1}(B-1) !}{\pi^{B}} \delta^{B}\left(\boldsymbol{a}_{+}-\boldsymbol{a}_{-}^{*}\right) \delta\left(\frac{1}{2}-\left\|\boldsymbol{a}_{+}\right\|^{2}\right)
$$

is the unique model which respects $\boldsymbol{a}_{+}=\boldsymbol{a}_{-}^{*}$, the normalization $\|\boldsymbol{a}\|^{2}=1=2\left\|\boldsymbol{a}_{+}\right\|^{2}$, and is invariant under the generalized orthogonal transformations (50). It gives the 
predictions

$$
\begin{aligned}
& M_{\mathrm{CO}, \alpha, q}=\frac{q !(B-1) !}{2^{q}(B+q-1) !}=\frac{q !}{(2 B)^{q}}\left(1-\frac{q(q-1)}{2 B}+O\left(B^{-2}\right)\right) \\
& C_{\mathrm{CO}, \alpha, \alpha^{\prime}}=\frac{1+\delta_{\alpha \alpha^{\prime}}+\delta_{\alpha \hat{\alpha}^{\prime}}}{4 B(B+1)}=\frac{1+\delta_{\alpha \alpha^{\prime}}+\delta_{\alpha \hat{\alpha}^{\prime}}}{(2 B)^{2}}\left(1-\frac{1}{B}+O\left(B^{-2}\right)\right) .
\end{aligned}
$$

The only difference in the leading order for large graphs is the term $\delta_{\alpha \hat{\alpha}^{\prime}}$ which ensures that the intensity correlation matrix is invariant under $\alpha \mapsto \hat{\alpha}$. Note that the deviations in the next order are twice as large in the orthogonal case.

In the limit $B \rightarrow \infty$ one may again replace the Circular Random Wave Model by a Gaussian Random Wave Model with the joint probability density

$$
\varphi_{\mathrm{GO}}(\boldsymbol{a}) \equiv \frac{B^{B}}{\pi^{B}} \delta^{B}\left(\boldsymbol{a}_{+}-\boldsymbol{a}_{-}^{*}\right) e^{-2 B\left\|\boldsymbol{a}_{+}\right\|^{2}}
$$

where only one half of the coefficients is taken from a Gaussian ensemble while the other half remains fixed by the symmetry constraints. The moments and the intensity correlation matrix in this Gaussian Random Wave Model are just the leading order terms from (52)

$$
M_{\mathrm{GO}, \alpha, q}=\frac{q !}{(2 B)^{q}} \quad \text { and } \quad C_{\mathrm{GO}, \alpha, \alpha^{\prime}}=\frac{1+\delta_{\alpha \alpha^{\prime}}+\delta_{\alpha \hat{\alpha}^{\prime}}}{(2 B)^{2}} .
$$

Note that the unitary and orthogonal universal Gaussian Random Wave Models (48) and (53) do not obey the normalization condition $\|\boldsymbol{a}\|^{2}=1$. In fact one has

$$
\left\langle\|\boldsymbol{a}\|^{2}\right\rangle_{\mathrm{GU}}=\left\langle\|\boldsymbol{a}\|^{2}\right\rangle_{\mathrm{GO}}=1
$$

only as an average property while the variances

$$
\left\langle\left(\|\boldsymbol{a}\|^{2}-1\right)^{2}\right\rangle_{\mathrm{GU}}=\frac{1}{2 B} \quad \text { and } \quad\left\langle\left(\|\boldsymbol{a}\|^{2}-1\right)^{2}\right\rangle_{\mathrm{GO}}=\frac{1}{B}
$$

are positive. Similarly, $\left|a_{\alpha}^{v}\right|$ cannot exceed one while the Gaussian Random Wave Models have a finite probability for this event. The Circular Random Wave Models take all these constraints into account correctly.

There is another obstruction to all the Random Waves Models (46), (48), (51) and (53). The matching conditions at vertex $i$ impose some correlation between the amplitudes supported on the neighboring bonds. This type of local and system-dependent correlations is ignored in the universal Random Wave Models. The most striking example consists in adding a Neumann vertex on some bond $b$ of an ergodic graph. By doing so, the bond $b$ is split into two new bonds $b_{1}$ and $b_{2}$, which can be oriented such that $\left(b_{1},+\right) \rightarrow\left(b_{2},+\right)$. Then, the Neumann condition imposes $\left|a_{b_{1}+}\right|^{2}=\left|a_{b_{2}+}\right|^{2}$ and $\left|a_{b_{1}-}\right|^{2}=\left|a_{b_{2}-}\right|^{2}$. These strong correlations contradict the predictions (47) and (52). Hence, a necessary condition for the universal Gaussian models (48) and (53) to be fulfilled in the limit of large graphs is that all the valencies tend to infinity.

For a finite graph one should expect that none of these models reproduces the exact correlation functions. Indeed, any numerical evaluation of the wave function statistics 
shows (amongst other things) an intensity fluctuation matrix that is far less uniform than the predictions from the Random Wave Models (see Figure1). The deviations can only be expected to vanish as $B \rightarrow \infty$ and if certain other conditions that we are going to derive are also satisfied.

\subsection{Asymptotic Quantum Ergodicity}

Let $G$ be a metric graph with $B$ bonds. An observable on $G$ is a family

$$
V=\left\{V_{b} \in C^{0}\left(\left[0, L_{b}\right]\right) \mid b \in \mathbb{N}_{B}\right\}
$$

of $B$ real functions $V_{b}(x)$ defined on the bonds of $G$. The mean value $\bar{V}$ of an observable $V$ is defined by

$$
\bar{V} \equiv \frac{2}{\operatorname{tr} L} \int_{G}^{\oplus} V \equiv \frac{2}{\operatorname{tr} L} \sum_{b=1}^{B} \int_{0}^{L_{b}} V_{b}(x) d x .
$$

Notice that $\frac{\operatorname{tr} L}{2}=\int_{G}^{\oplus} 1$ is the volume of $G$. If an observable $V$ is constant on each bond, one can simply write $V=\left(V_{b}\right)_{b \in \mathbb{N}_{B}}$ with $V_{b} \in \mathbb{R}$. The mean value of such an observable reads

$$
\bar{V}=\frac{\sum_{b=1}^{B} V_{b} L_{b}}{\sum_{b=1}^{B} L_{b}}
$$

and is invariant under a global scaling of the bond lengths.

Suppose now that $S \in U(2 B)$ is a scattering matrix on $G$. The quantum graph $(G, S)$ is said to be quantum ergodic if and only if there exists a subsequence $i \mapsto v(i)$ of density 1 such that

$$
\lim _{i \rightarrow \infty} \frac{\left(\Psi^{v(i)}, V \Psi^{v(i)}\right)}{\left(\Psi^{v(i)}, \Psi^{v(i)}\right)}=\bar{V}
$$

for any observable $V$. In this definition, $\Psi^{v}=\bigoplus_{b=1}^{B} \psi_{b}^{v}$ denotes an eigenfunction of $H$ of eigenvalue $k_{v}^{2}$. By assumption, it is unique up to multiplication by complex numbers (or by real numbers for the orthogonal class).

The left-hand side of 60 represents the mean value of the observable $V$ in the eigenstate $\Psi^{v(i)}$. A straightforward calculation shows that

$$
\begin{aligned}
\left(\Psi^{v}, V \Psi^{v}\right)= & \sum_{b=1}^{B}\left(\left|a_{b+}^{v}\right|^{2}+\left|a_{b-}^{v}\right|^{2}\right) \int_{0}^{L_{b}} V_{b}(x) d x \\
& +2 \Re \sum_{b=1}^{B} a_{b-}^{\nu^{*}} a_{b+}^{v} \int_{0}^{L_{b}} V_{b}(x) e^{2 i k_{v}\left(x-\frac{L_{b}}{2}\right)} d x
\end{aligned}
$$

for the wave function $\Psi^{v}$ with wave number $k_{v}>0$ and amplitudes $a_{b+}^{v}$ and $a_{b-}^{v}$ as in (12). Since the observable $V$ is assumed continuous on each bond, and since $\left|a_{b-}^{v *} a_{b+}^{v}\right| \leq$ 1 , the second term in the right-hand side of (61) is $O\left(k_{v}^{-1}\right)$. In the high energy limit this second term gives no contribution to the left-hand side of (60). Moreover, the first term in the right-hand side of 61 remains unchanged if the observable $V$ is replaced with 
the observable $W$ defined by $W_{b} \equiv L_{b}^{-1} \int_{0}^{L_{b}} V_{b}(x) d x$. These two remarks imply that, in the definition (60) of quantum ergodicity, it is sufficient to consider observables that are constant on each bond, and this will always be the case in what follows.

If the equality (60) holds for any observable of vanishing mean $\bar{V}=0$, then it also holds for any observable $W$. In order to see this, it is sufficient to observe that $W-\bar{W}$ has vanishing mean and to apply (60) to this new observable. Hence, without loss of generality, one can also restrict attention to observables $V$ with $\bar{V}=0$.

If the identity (60) is satisfied for any subsequence of eigenfunctions, the quantum graph is said to be quantum unique ergodic. In [38], it is shown that many short closed cycles, like the triangle $\beta_{1} \rightarrow \beta_{2} \rightarrow \beta_{3} \rightarrow \beta_{1}$ for instance, support eigenfunctions with arbitrarily high energies. These eigenfunctions, called scars, break quantum unique ergodicity. While these scarred eigenfunctions were obtained explicitly for Neumann quantum graphs, quantum unique ergodicity should certainly not be expected to hold on general finite quantum graphs.

Moreover, quantum ergodicity is generally not realized on a finite quantum graph as well. This notion has thus to be replaced with a weaker one which we call asymptotic quantum ergodicity. Let us consider an infinite sequence $\left\{\left(G_{l}, S_{l}\right)\right\}_{l \in \mathbb{N}}$ of quantum graphs with increasing number of bonds $B_{l}<B_{l+1}$. We also suppose that the bonds of any $G_{l}$ have bond lengths that satisfy

$$
L_{b} \in\left[L_{\min }, L_{\max }\right] \quad \text { where } \quad 0<L_{\min }<L_{\max }<\infty
$$

are independent of $l$. Such a sequence will be called increasing. We always assume that either all the graphs $\left(G_{l}, S_{l}\right)$ are time-reversal invariant, or they all break this symmetry. The eigenfunctions of $\left(G_{l}, S_{l}\right)$ are denoted by $\Psi_{l}^{v}$, and similarly, all the quantities introduced above are indexed by $l$. Besides, a sequence $\left\{V_{l}\right\}_{l \in \mathbb{N}}$, where $V_{l}$ is an observable on $G_{l}$, is said to be acceptable if and only if the two conditions

$$
\begin{gathered}
\lim _{l \rightarrow \infty} \bar{V}_{l} \equiv \bar{V}_{\infty} \text { exists } \\
0 \leq\left|V_{l, b}\right| \leq V_{\max }
\end{gathered}
$$

are fulfilled. Then, an increasing sequence $\left\{\left(G_{l}, S_{l}\right)\right\}_{l \in \mathbb{N}}$ of quantum graphs is said to be asymptotically quantum ergodic if and only if

$$
\lim _{l \rightarrow \infty} \lim _{i \rightarrow \infty} \frac{\left(\Psi_{l}^{\nu(i)}, V_{l} \Psi_{l}^{v(i)}\right)}{\left(\Psi_{l}^{\nu(i)}, \Psi_{l}^{v(i)}\right)}=\bar{V}_{\infty}
$$

for all acceptable sequences of observables $\left\{V_{l}\right\}_{l \in \mathbb{N}}$. The limit $l \rightarrow \infty$ plays the role of the semiclassical limit for quantum graphs.

For the sequences of graphs satisfying [64], the rate of convergence is also of particular interest. Therefore, we will treat a single finite quantum graph first, and come back to convergence and rate considerations afterwards.

A calculation similar to (61) shows that, for an observable $V$ on $G$ constant on each bond, one has

$$
\left(\Psi^{v}, V \Psi^{v}\right)=\left\langle\boldsymbol{a}^{v}\left|V L\left(1+\frac{\sin \left(k_{v} L\right)}{k_{v} L}\right)\right| \boldsymbol{a}^{v}\right\rangle=\left\langle\boldsymbol{a}^{v}|V L| \boldsymbol{a}^{v}\right\rangle+O\left(k_{v}^{-1}\right),
$$


where $\left|\boldsymbol{a}^{v}\right\rangle \in \mathcal{A}$ is the vector of amplitudes defining $\Psi^{v}$ through the construction (12). There is a slight abuse of notation in this expression. On the left-hand side, $V=$ $\left(V_{b}\right)_{b \in \mathbb{N}_{B}}$ is an observable constant on each bond, whereas on the right-hand side $V$ stands for the diagonal $2 B \times 2 B$ matrix $V_{b d, b^{\prime} d^{\prime}} \equiv \delta_{b, b^{\prime}} \delta_{d, d^{\prime}} V_{b}$. Such a matrix is called observable on $\mathcal{A}$ and has mean value

$$
\bar{V} \equiv \frac{\operatorname{tr}(V L)}{\operatorname{tr} L}=\frac{\sum_{\beta=1}^{2 B} V_{\beta} L_{\beta}}{\sum_{\beta=1}^{2 B} L_{\beta}}=\frac{\sum_{b=1}^{B} V_{b} L_{b}}{\sum_{b=1}^{B} L_{b}} .
$$

This expression coincides with the mean value 59 of $V$ seen as an observable on $G$ constant on each bond.

From 60, 65) and (66), we deduce that a quantum graph is quantum ergodic if and only if there exists a subsequence $i \mapsto v(i)$ of density 1 such that

$$
\lim _{i \rightarrow \infty} \frac{\langle V L\rangle_{v(i)}}{\langle L\rangle_{\nu(i)}} \equiv \lim _{i \rightarrow \infty} \frac{\left\langle\boldsymbol{a}^{\nu(i)}|V L| \boldsymbol{a}^{\nu(i)}\right\rangle}{\left\langle\boldsymbol{a}^{v(i)}|L| \boldsymbol{a}^{v(i)}\right\rangle}=\bar{V}
$$

for any observable $V$ on $\mathcal{A}$. As above, one can restrict attention to observables $V$ such that $\bar{V}=0$ without loss of generality.

A standard theorem of ergodic theory, proven for example in [45], states that the quantum ergodicity property 67 is equivalent to the vanishing of

$$
F_{V} \equiv \lim _{K \rightarrow \infty} \frac{1}{N(K)} \sum_{k_{v} \leq K} \frac{\langle V L\rangle_{v}^{2}}{\langle L\rangle_{v}^{2}}
$$

for all observables $V$ on $\mathcal{A}$ with $\bar{V}=0$. Moreover, since the bond lengths are bounded by $L_{\min }$ and $L_{\max }$ by assumption (62), this property is also equivalent to the vanishing of the fluctuations

$$
\mathcal{F}_{V} \equiv\left(\frac{2 B}{\operatorname{tr} L}\right)^{2} \sum_{\beta, \beta^{\prime}=1}^{2 B}(V L)_{\beta}(V L)_{\beta^{\prime}} C_{\beta \beta^{\prime}}
$$

for all observables $V$ on $\mathcal{A}$ with $\bar{V}=0$, where the intensity correlation matrix $C_{\beta \beta^{\prime}}$ in the right-hand side is defined in (44).

In the case of an increasing sequence of graphs $\left\{\left(G_{l}, S_{l}\right)\right\}_{l \in \mathbb{N}}$, asymptotic quantum ergodicity is obeyed if and only if the sequence $\left\{F_{l, V_{l}}\right\}_{l \in \mathbb{N}}$ whose terms are defined as in (68), or equivalently the sequence $\left\{\mathcal{F}_{l, V_{l}}\right\}_{l \in \mathbb{N}}$ whose terms are defined as in 69, converges to zero as $l \rightarrow \infty$ for all acceptable sequences of observables $\left\{V_{l}\right\}_{l \in \mathbb{N}}$. The rate of convergence is then called the rate of quantum ergodicity.

The Gaussian Random Wave Models (48) and (53) predict the fluctuations

$$
\mathcal{F}_{V}=\bar{V}^{2}+\kappa \frac{\operatorname{tr}(V L)^{2}}{(\operatorname{tr} L)^{2}},
$$

as can easily be shown from the Gaussian predictions for the intensity correlation matrix (49) and (54). The parameter $\kappa$ was defined in (28). The term proportional to $\kappa$ describes the deviation from quantum ergodicity. For any admissible observable and bond lengths bounded by (62) the deviation predicted by the Gaussian Random Wave 
Models is $O\left(B^{-1}\right)$. Hence the Gaussian Random Wave Models predict that any increasing sequence of quantum graphs is asymptotically quantum ergodic and that the rate of convergence is larger by a factor of two if time-reversal symmetry is conserved.

Note, that quantum ergodicity holds on average, in the sense that

$$
A_{V} \equiv \lim _{K \rightarrow \infty} \frac{1}{N(K)} \sum_{k_{v} \leq K} \frac{\langle V L\rangle_{v}}{\langle L\rangle_{v}}=\bar{V}
$$

for all observables $V$. This is known as the local Weyl law. It is easily checked to hold for any quantum graph. Indeed, by the definition (42), $A_{V}$ can also be written

$$
A_{V}=\frac{2 B}{\operatorname{tr} L} \sum_{\beta=1}^{2 B}(V L)_{\beta}\left\langle\left|a_{\beta}\right|^{2}\right\rangle
$$

Then, the identity (41) shows that $\left\langle\left|a_{\beta}\right|^{2}\right\rangle=(2 B)^{-1}$, and the definition of $\bar{V}$ concludes the proof of the claim. The restriction to incommensurable bond lengths is not necessary for the local Weyl law, indeed we will show in the following subsection that 41] is true for any choice of bond lengths.

\subsection{Green Matrices and Trace Formulae}

For $(G, S)$ a quantum graph, and for $\epsilon>0$, one defines a sub-unitary quantum map $U_{\epsilon}(k)$ by

$$
U_{\epsilon}(k)=T(k) S_{\epsilon} T(k), \quad \text { with } \quad S_{\epsilon} \equiv e^{-\epsilon} S,
$$

and where $T(k)$ is the propagation matrix of $G$ given in (16). The retarded Green matrix (resolvent) $G(k)$ is the matrix-valued function on $\mathbb{R}_{+}$defined by

$$
G(k) \equiv\left(1-U_{\epsilon}(k)\right)^{-1}=\sum_{n=1}^{2 B} \frac{|n, k\rangle\langle n, k|}{1-e^{i\left(\phi_{n}(k)+i \epsilon\right)}} .
$$

It has poles in the lower complex half-plane at $\phi_{n}(k)=2 \pi p-i \epsilon$ for any $p \in \mathbb{Z}$. The advanced Green matrix $G^{\dagger}(k)$ is the hermitian conjugate of $G(k)$, that is

$$
G^{\dagger}(k)=\left(1-U_{\epsilon}^{\dagger}(k)\right)^{-1}=\sum_{n=1}^{2 B} \frac{|n, k\rangle\langle n, k|}{1-e^{-i\left(\phi_{n}(k)-i \epsilon\right)}} .
$$

It has poles in the upper complex half-plane at $\phi_{n}(k)=2 \pi p+i \epsilon$ for any $p \in \mathbb{Z}$. Making use of formula (25), it is not difficult to check that, for any integer $q \geq 2$, and for any permutation $\sigma \in S_{q}$, the statistical quantities defined in 37 with $p=q$ read

$$
\left\langle a_{\beta_{0}}^{*} \ldots a_{\beta_{q-1}}^{*} a_{\beta_{0}^{\prime}} \ldots a_{\beta_{q-1}^{\prime}}\right\rangle=\lim _{\epsilon \rightarrow 0} \frac{(2 \epsilon)^{q-1}}{2 B}\left\langle\prod_{j=1}^{q-1} G(k)_{\beta_{\sigma(j)} \beta_{j}^{\prime}} \cdot G^{\dagger}(k)_{\beta_{\sigma(0)} \beta_{0}^{\prime}}\right\rangle_{k},
$$

where, in the right-hand side, the average over $k$ is defined by the formula

$$
\langle f(k)\rangle_{k} \equiv \lim _{K \rightarrow \infty} \frac{1}{K} \int_{0}^{K} f(k) d k
$$


which is meaningful for any function $f$ integrable on every compact interval $[0, K]$. The formula (76) relies on the non-degeneracy of the spectrum, which generically follows from the incommensurability of the bond lengths. However, it still holds if the subsequence of levels $k_{v}$ that are degenerate is of density zero. There are other versions of the equality (76) where the right-hand side involve $n_{r} \in \mathbb{N}$ elements of $G(k)$ and $n_{a} \in \mathbb{N}$ elements of $G^{\dagger}(k)$ with $n_{r}+n_{a}=q$. A formula similar to (76) is used in [20] to study the statistical properties of the eigenfunctions in disordered systems. For the derivation of exact expressions the choice of the permutation $\sigma \in S_{q}$ in (76) is mainly a matter of computational ease (and sometimes taste). Throughout the remainder of this subsection we will show how the different choices lead to different exact expressions.

The Green matrices $G(k)$ and $G^{\dagger}(k)$ can be viewed as the results of summing geometrical series in $U_{\epsilon}(k)$ and $U_{\epsilon}^{\dagger}(k)$. This gives rise to interpretations of their components as sums of walks on the quantum graph $(G, S)$. An oriented walk $\vec{\beta}$ is a list $\left(\beta_{0}, \beta_{1}, \ldots, \beta_{n}\right)$ of consecutive directed bonds on the graph. Its topological length $|\vec{\beta}|$ is the number of vertices traversed, that is $|\vec{\beta}|=n$. The set of all oriented walks having topological length $n$ is written $W_{n}$. The metric length of $\vec{\beta}$ is

$$
l(\vec{\beta}) \equiv \frac{L_{\beta_{0}}}{2}+\sum_{i=1}^{n-1} L_{\beta_{i}}+\frac{L_{\beta_{n}}}{2}
$$

The origin and terminus of $\vec{\beta}$ are respectively $o \vec{\beta} \equiv \beta_{0}$ and $t \vec{\beta} \equiv \beta_{n}$. The set of walks in $W_{n}$ having origin $\beta$ and terminus $\beta^{\prime}$ is written $W_{n}\left(\beta, \beta^{\prime}\right)$, and $\cup_{n \in \mathbb{N}_{0}} W_{n}\left(\beta, \beta^{\prime}\right) \equiv W\left(\beta, \beta^{\prime}\right)$. We also define the stability amplitude

$$
A_{\vec{\beta}} \equiv \prod_{i=0}^{n-1} S_{\beta_{i+1} \beta_{i}} .
$$

With these definitions, it is easy to see that

$$
G(k)_{\beta \beta^{\prime}}=\sum_{\vec{\beta} \in W\left(\beta^{\prime}, \beta\right)} e^{-\epsilon|\vec{\beta}|} e^{i k l(\vec{\beta})} A_{\vec{\beta}}
$$

and

$$
G^{\dagger}(k)_{\beta \beta^{\prime}}=\sum_{\vec{\beta} \in W\left(\beta, \beta^{\prime}\right)} e^{-\epsilon|\vec{\beta}|} e^{-i k l(\vec{\beta})} A_{\vec{\beta}}^{*} .
$$

Together with (76), these formulae enable one to express the autocorrelation functions $C_{[\alpha]}$ in (42) as sums over oriented walks.

The different choices for the order of the left indices $\beta$ in (76) lead to different equivalent expressions for the autocorrelation functions $C_{[\alpha]}$ in terms of oriented walks. In general, showing the equivalence between these trace formulae at the level of oriented walks turns out to be a very difficult problem. In this subsection, these non-trivial equivalences are illustrated by two alternative proofs of the local Weyl law 711.

In the case of the intensity correlation matrix $C_{\beta \beta^{\prime}}$, two permutations $\sigma \in S_{2}$ of the 


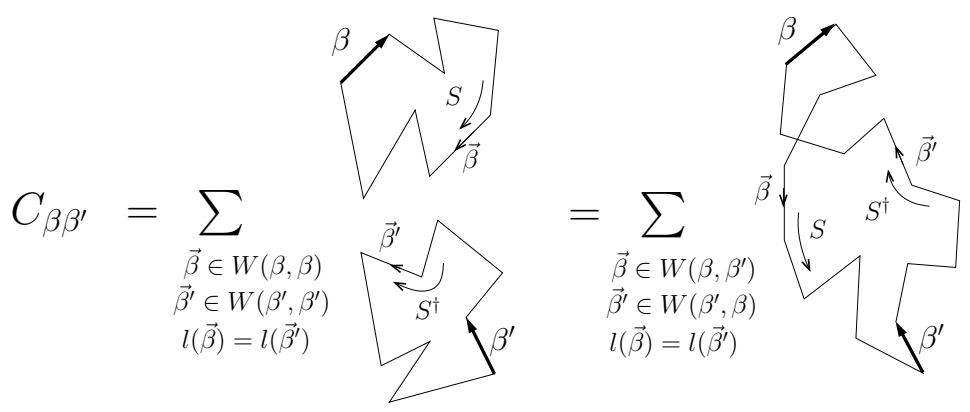

Figure 2: The two equivalent formulae 83 and 85 for the autocorrelation function $C_{\beta \beta^{\prime}}$. The underlying graph has not been represented for sake of clarity. The trace formulae 88 and 89 ) are obtained from the ones represented here by adding the contributions where $S$ and $S^{\dagger}$ are swapped and by dividing by two.

left indices in (76) can be chosen. The identity permutation $\sigma=$ id leads to

$$
\begin{aligned}
C_{\beta \beta^{\prime}} & =\lim _{\epsilon \rightarrow 0} \frac{\epsilon}{B}\left\langle G(k)_{\beta \beta} G^{\dagger}(k)_{\beta^{\prime} \beta^{\prime}}\right\rangle_{k} \\
& =\lim _{\epsilon \rightarrow 0} \frac{\epsilon}{B} \sum_{\vec{\beta} \in W(\beta, \beta)} \sum_{\overrightarrow{\beta^{\prime}} \in W\left(\beta^{\prime}, \beta^{\prime}\right)} e^{-\epsilon\left(|\vec{\beta}|+\left|\vec{\beta}^{\prime}\right|\right)} \delta_{l(\vec{\beta}), l\left(\overrightarrow{\beta^{\prime}}\right)} A_{\vec{\beta}} A_{\vec{\beta}^{\prime}}{ }^{*}
\end{aligned}
$$

while choosing the transposition $\sigma=\left(\begin{array}{ll}1 & 2\end{array}\right)$ leads to

$$
\begin{aligned}
C_{\beta \beta^{\prime}} & =\lim _{\epsilon \rightarrow 0} \frac{\epsilon}{B}\left\langle G(k)_{\beta^{\prime} \beta} G^{\dagger}(k)_{\beta \beta^{\prime}}\right\rangle_{k} \\
& =\lim _{\epsilon \rightarrow 0} \frac{\epsilon}{B} \sum_{\vec{\beta} \in W\left(\beta, \beta^{\prime}\right)} \sum_{\overrightarrow{\beta^{\prime}} \in W\left(\beta, \beta^{\prime}\right)} e^{-\epsilon\left(\overrightarrow{\mid}|+| \overrightarrow{\beta^{\prime}} \mid\right)} \delta_{l(\vec{\beta}), l\left(\overrightarrow{\beta^{\prime}}\right)} A_{\vec{\beta}^{\prime}} A_{\vec{\beta}^{\prime}}{ }^{*}
\end{aligned}
$$

In both cases, the Kronecker symbols originate from the average over $k$.

These orbit expressions can also be recovered by means of the Poisson summation formula. If $\delta_{\epsilon}(x)$ denotes the Lorentzian of width $\epsilon$ centered at the origin, this formula leads to

$$
\begin{aligned}
\mathcal{G}_{\epsilon}(k) & \equiv \sum_{n=1}^{2 B}|n, k\rangle\langle n, k| \sum_{p=0}^{\infty} \delta_{\epsilon}\left(\phi_{n}(k)-2 \pi p\right) \\
& =\frac{1}{2 \pi} \mathbb{1}+\frac{1}{2 \pi} \sum_{q=1}^{\infty}\left(U(k)^{q}+U^{\dagger}(k)^{q}\right) e^{-\epsilon q}
\end{aligned}
$$

The trace formula 83 , or more exactly its symmetrization obtained by replacing $A_{\vec{\beta}} A_{\vec{\beta}^{\prime}}{ }^{*}$ with $\frac{1}{2}\left(A_{\vec{\beta}} A_{\vec{\beta}^{\prime}}{ }^{*}+A_{\vec{\beta}}{ }^{*} A_{\vec{\beta}^{\prime}}\right)$, follows from 87 and the identity

$$
C_{\beta \beta^{\prime}}=\frac{2 \pi^{2} \epsilon}{B}\left\langle\mathcal{G}_{\epsilon}(k)_{\beta \beta} \mathcal{G}_{\epsilon}(k)_{\beta^{\prime} \beta^{\prime}}\right\rangle_{k}
$$


This identity is a consequence of the fact that, in terms of distributions, the product $2 \pi \epsilon \delta_{\epsilon}(x) \delta_{\epsilon}(y)$ tends to zero if $x \neq y$ and to $\delta(x)$ if $x=y$. Similarly, the symmetric version of 85. follows from 87 and

$$
C_{\beta \beta^{\prime}}=\frac{2 \pi^{2} \epsilon}{B}\left\langle\mathcal{G}_{\epsilon}(k)_{\beta^{\prime} \beta} \mathcal{G}_{\epsilon}(k)_{\beta \beta^{\prime}}\right\rangle_{k} .
$$

The main advantage of the expressions (88) and (89), involving $\mathcal{G}_{\epsilon}(k)$, over their analogues (82) and 84, which involve $G(k)$, is that the matrix $\mathcal{G}_{\epsilon}(k)$ is real, whereas $G(k)$ and $G^{\dagger}(k)$ have non-vanishing imaginary parts and must always appear together in (76). In particular, the first moment $M_{\beta, 1}=\left\langle\left|a_{\beta}\right|^{2}\right\rangle$ can be written

$$
M_{\beta, 1}=\frac{\pi}{B} \lim _{\epsilon \rightarrow 0}\left\langle\mathcal{G}_{\epsilon}(k)_{\beta \beta}\right\rangle_{k},
$$

which involves a single closed oriented walk, while, in terms of matrices $G(k)$, an additional directed bond $\beta^{\prime}$ must first be introduced in order for $M_{\beta, 1}$ to be written as the sum $\sum_{\beta^{\prime}=1}^{2 B} C_{\beta \beta^{\prime}}=\sum_{\beta^{\prime}=1}^{2 B}\left\langle\left|a_{\beta}\right|^{2}\left|a_{\beta^{\prime}}\right|^{2}\right\rangle$ and the representations 83) or 855 to be used. From 87 and , one finds directly that

$$
M_{\beta, 1}=\frac{1}{2 B}
$$

which, together with (72), provides a second proof of the local Weyl law. Let us now use the trace formula (85) and perform the sum over the directed bond $\beta^{\prime}$. It is easy to show by induction over $n$ and $m$ that the unitarity of the scattering matrix $S$ implies

$$
\sum_{\beta^{\prime}=1}^{2 B} \sum_{\vec{\beta} \in W_{n}\left(\beta, \beta^{\prime}\right)} \sum_{\overrightarrow{\beta^{\prime}} \in W_{m}\left(\beta, \beta^{\prime}\right)} \delta_{l(\vec{\beta}), l\left(\vec{\beta}^{\prime}\right)} A_{\vec{\beta}} A_{\vec{\beta}^{\prime}}^{*}=\delta_{n, m}
$$

for all $n, m \in \mathbb{N}_{0}$. With 85 , this gives

$$
M_{\beta, 1}=\sum_{\beta^{\prime}=1}^{2 B} C_{\beta \beta^{\prime}}=\lim _{\epsilon \rightarrow 0} \frac{\epsilon}{B} \sum_{n, m=0}^{\infty} e^{-\epsilon(n+m)} \delta_{n, m}=\frac{1}{2 B}
$$

which, together with 72, yields a third proof of the local Weyl law.

The choice for the permutation $\sigma \in S_{q}$ in (76) leads, in the case $q=2$, to the equivalent expressions 83 and 85 for $C_{\beta \beta^{\prime}}$ in terms of oriented walks that are illustrated in Figure 2. Similar pictures could also be drawn for $C_{\left[\beta_{0}, \ldots \beta_{q-1}\right]}$ when $q>2$. Indeed, the right-hand side of (76) with $\beta_{j}^{\prime}=\beta_{j}$ for all $0 \leq j \leq q-1$ and with a fixed permutation $\sigma \in S_{q}$ can be expressed as a sum over $q$ oriented walks $\vec{\beta}_{1}, \ldots, \vec{\beta}_{q}$, where each $\vec{\beta}_{j}$ leads from the directed bond $\beta_{j}$ to the directed bond $\beta_{\sigma(j)}$. The walk $\vec{\beta}_{q}$ is followed with $S_{\epsilon}^{\dagger}$, whereas the $q-1$ other walks are followed with $S_{\epsilon}$, and its metric length must equal the sum of the metric lengths of the $q-1$ other walks. Therefore, (76) yields $q \cdot q$ ! different ways of expressing the autocorrelation function $C_{[\beta]}$ of degree $q$ in terms of oriented walks. Here, the first factor $q$ accounts for the $q$ possible choices for the walk followed with $S_{\epsilon}^{\dagger}$. 


\subsection{Long Diagonal Orbits}

An expression for the fluctuations $\mathcal{F}_{V}$ in 69 can be obtained by retaining only a subset of the whole set of pairs of oriented walks $\left(\vec{\beta}, \vec{\beta}^{\prime}\right)$ entering 83 . For this purpose, it is convenient to come back to the expression 188 of $C_{\beta \beta^{\prime}}$ in terms of $\mathcal{G}_{\epsilon}(k)$ and write the fluctuations of an observable $V$ with $\bar{V}=0$ as

$$
\mathcal{F}_{V}=\frac{8 B \pi^{2} \epsilon}{(\operatorname{tr} L)^{2}}\left\langle\left(\operatorname{tr}\left(\mathcal{G}_{\epsilon}(k) V L\right)\right)^{2}\right\rangle_{k} .
$$

The right-hand side can be written in terms of periodic orbits rather than closed oriented walks as in 83). A periodic orbit is an equivalence class of closed oriented walks whose sequences of directed bonds differ from each other by cyclic permutations. For a periodic orbit $p$, the notions of reverse $\hat{p}$, topological length $|p|$, metric length $l_{p}$ and stability amplitude $A_{p}$ are inherited from the oriented walks terminology, and the repetition number $r_{p}$ is the number of times $p$ retraces itself. With this notation, one gets from 87

$$
\operatorname{tr}\left(G_{\epsilon} V L\right)=\frac{1}{\pi} \mathfrak{R} \sum_{p} e^{-\epsilon|p|} \frac{(V L)_{p}}{r_{p}} e^{i k l_{p}} A_{p}
$$

where the sum is over all the periodic orbits on the graph and $(V L)_{p}$ stands for the number obtained by accumulating the values $(V L)_{\beta}$ of $V L$ along $p$. The square of the last formula admits the spectral average

$$
\left\langle\left[\operatorname{tr}\left(G_{\epsilon} V L\right)\right]^{2}\right\rangle_{k}=\frac{1}{2 \pi^{2}} \sum_{p, q: l_{p}=l_{q}} \frac{(V L)_{p}(V L)_{q}}{r_{p} r_{q}} \mathfrak{R}\left(A_{p} A_{q}^{*}\right) e^{-\epsilon(|p|+|q|)}
$$

The diagonal approximation, which consists in only keeping the pairs $q=p$ and $q=\hat{p}$ in the time-reversal invariant case, yields

$$
\left\langle\left[\operatorname{tr}\left(G_{\epsilon} V L\right)\right]^{2}\right\rangle_{k}^{\operatorname{diag}}=\frac{\kappa}{2 \pi^{2}} \sum_{p, q: l_{p}=l_{q}} \frac{\left[(V L)_{p}\right]^{2}}{r_{p}^{2}}\left|A_{p}\right|^{2} e^{-2 \epsilon|p|}
$$

where $\kappa$ is the parameter as in (70) indicating whether time-reversal invariance is broken or conserved. We have neglected some corrections in the diagonal approximation which are due to repetitions and self-retracing orbits. These can be shown not to contribute in the present context. The formula (97) is then approximated further. The orbits for which $r_{p}>1$ are rare, so that we only keep the primitive orbits, namely those with $r_{p}=1$. We also take the long orbits approximation [18], which amounts to approximating

$$
\left[(V L)_{p}\right]^{2} \approx\left[(V L)^{2}\right]_{p} \approx|p| \frac{\operatorname{tr}(V L)^{2}}{2 B} .
$$

Besides, the stability amplitude is known to behave like [25]

$$
\left|A_{p}\right|^{2} \sim e^{-\alpha|p|}
$$


where $\alpha$ is the topological entropy. This parameter also characterizes the number $|p|^{-1} e^{\alpha|p|}$ of periodic orbits having topological length $|p|$. With all these approximations, (97) reduces to the integral

$$
\left\langle\left[\operatorname{tr}\left(\mathcal{G}_{\epsilon} V L\right)\right]^{2}\right\rangle_{k}^{\mathrm{diag}} \approx \frac{\kappa}{2 \pi^{2}} \int_{0}^{\infty} \frac{e^{\alpha|p|} d|p|}{|p|} \cdot|p| \frac{\operatorname{tr}(V L)^{2}}{2 B} \cdot e^{-\alpha|p|} \cdot e^{-2 \epsilon|p|} .
$$

Hence

$$
\mathcal{F}_{V}^{\text {diag }} \approx \kappa \frac{\operatorname{tr}(V L)^{2}}{(\operatorname{tr} L)^{2}} .
$$

This formula, obtained from the long diagonal orbits, coincides with the prediction (70) of the Gaussian Random Wave Models (48) and (53). It predicts asymptotic quantum ergodicity for any increasing sequence of quantum graphs and a universal rate of convergence $B^{-1}$, as in [18].

\section{Generating Functions}

\subsection{Definition and Principles}

The Green matrices introduced in the subsection 3.4 can be obtained as the derivatives of certain determinants. It is convenient first to introduce a Grassmann algebra $\Lambda$, which can be decomposed as the direct sum of its commuting sub-algebra $\Lambda_{B}$, called bosonic, and a set $\Lambda_{F}$ of elements anticommuting with each other, called fermionic. Then, the amplitude space $\mathcal{A}$ can be graded to get $\mathcal{A} \oplus \mathcal{A}$, and the Grassmann envelope $(\mathcal{A} \oplus \mathcal{A})(\Lambda)$ defined as in [5] can be built. This set reads

$$
(\mathcal{A} \oplus \mathcal{A})(\Lambda) \equiv\left\{V=\left(\begin{array}{c}
V_{B} \\
V_{F}
\end{array}\right) ; V_{B / F}=\sum_{\beta=1}^{2 B} V_{B / F}^{\beta}\left|e_{\beta}\right\rangle, V_{B / F}^{\beta} \in \Lambda_{B / F}\right\},
$$

where the elements $\left|e_{\beta}\right\rangle$ refer to the elements in (11) of the natural basis of $\mathcal{A}$. The elements of $(\mathcal{A} \oplus \mathcal{A})(\Lambda)$ are called supervectors. The set of endomorphisms on (102), once written in the natural basis of $\mathcal{A}$, form a set of supermatrices written $L(\mathcal{A} \mid \mathcal{A})$. For $q \geq 2$ an integer, let us introduce complex numbers $j_{1}, \ldots, j_{q-1}$ and $j_{0}$, respectively referred to as retarded and advanced sources, and let us also consider $q$ directed bonds $\alpha_{1}, \ldots \alpha_{q-1}$ and $\alpha_{0}$. The corresponding retarded and advanced source supermatrices are defined by

$$
\begin{gathered}
J_{r}\left(\boldsymbol{j}_{r}\right) \equiv 1+E_{B} \otimes \boldsymbol{j}_{r} \boldsymbol{E}^{(r)}, \\
J_{a}\left(j_{a}\right) \equiv 1+E_{B} \otimes j_{a} E^{(a)},
\end{gathered}
$$

where $E_{B}$ is the projector onto the bosonic sector of $(\mathcal{A} \oplus \mathcal{A})(\Lambda)$,

$$
\boldsymbol{j} \equiv\left(\begin{array}{c}
j_{a} \\
\boldsymbol{j}_{\boldsymbol{r}}
\end{array}\right) \equiv\left(\begin{array}{c}
j_{0} \\
j_{1} \\
\vdots \\
j_{q-1}
\end{array}\right), \quad \boldsymbol{E} \equiv\left(\begin{array}{c}
E^{(a)} \\
\boldsymbol{E}^{(r)}
\end{array}\right) \equiv\left(\begin{array}{c}
E^{\alpha_{0}, \alpha_{0}} \\
E^{\alpha_{1}, \alpha_{1}} \\
\vdots \\
E^{\alpha_{q-1}, \alpha_{q-1}}
\end{array}\right),
$$


and, for any two directed bonds $\alpha, \alpha^{\prime} \in \mathbb{N}_{2 B}, E^{\alpha, \alpha^{\prime}}$ stands for the $2 B \times 2 B$ matrix whose components are $\left(E^{\alpha, \alpha^{\prime}}\right)_{\beta \beta^{\prime}} \equiv \delta_{\alpha, \beta} \delta_{\alpha^{\prime}, \beta^{\prime}}$ in the natural basis of $\mathcal{A}$. The number $q-1$ of retarded sources corresponds to the number of matrices $E^{\alpha_{j}, \alpha_{j}}$ contained in $\boldsymbol{E}^{(\boldsymbol{r})}$, so that the product in 103 makes sense. In 103, 104 and in what follows, some unit matrices or supermatrices are not explicitly written in order to keep the notation as simple as possible. For example, the symbols 1 in (103) and (104) must be read $\mathbb{1}_{B F} \otimes \mathbb{1}_{\mathcal{A}}$, where $\mathbb{1}_{B F}$ is the unit supermatrix in Bose-Fermi space and $\mathbb{1}_{\mathcal{A}}$ is the $2 B \times 2 B$ unit matrix in amplitude space $\mathcal{A}$.

Let $q \geq 2$ and let $[\alpha] \equiv\left[\alpha_{0}, \alpha_{1}, \ldots, \alpha_{q-1}\right]$ be a list of $q$ directed bonds. The corresponding generating function is defined by

$$
\xi_{[\alpha]}(\boldsymbol{j}) \equiv\left\langle\operatorname{sdet}^{-1}\left(1-J_{r}\left(\boldsymbol{j}_{\boldsymbol{r}}\right) \cdot U_{\epsilon}(k)\right)\left(1-J_{a}\left(j_{a}\right) \cdot U_{\epsilon}^{\dagger}(k)\right)\right\rangle_{k},
$$

where $J_{r}\left(\boldsymbol{j}_{\boldsymbol{r}}\right)$ and $J_{a}\left(j_{a}\right)$ are defined from $\boldsymbol{j} \equiv\left(j_{a}, \boldsymbol{j}_{\boldsymbol{r}}\right)^{T}=\left(j_{0}, j_{1}, \ldots, j_{q-1}\right)^{T}$ and from the directed bonds in $[\alpha]$ as in (103) and (104). Notice that this function is well defined in a neighborhood of the origin, and that it also reads

$$
\xi_{[\alpha]}(\boldsymbol{j})=\left\langle\operatorname{det}^{-1}\left(1-\boldsymbol{j}_{\boldsymbol{r}} \boldsymbol{E}^{(\boldsymbol{r})}(G(k)-1)\right)\left(1-j_{a} E^{(a)}\left(G^{\dagger}(k)-1\right)\right)\right\rangle_{k}
$$

in terms of Green matrices.

It is convenient at this point to give a general rule governing derivatives of determinants of the form 107). An important quantity is the $\rho$ factor

$$
\rho_{\alpha}(\sigma) \equiv \alpha^{\text {number of cycles in } \sigma}
$$

defined for any $\alpha \in \mathbb{R}$ and any permutation $\sigma \in S_{s}$ of $s \in \mathbb{N}$ elements. This factor can be seen as a generalization of the signature $(-1)^{\sigma}$ of $\sigma \in S_{s}$ since the identity $(-1)^{\sigma}=(-1)^{s} \rho_{-1}(\sigma)$ holds. Now, if $\boldsymbol{A}=\left(A^{(1)}, \ldots, A^{(s)}\right)^{T}$ is a vector containing $s \in \mathbb{N}$ square matrices $A^{(i)}$ of size $n \in \mathbb{N}$ and if $j \in \mathbb{C}^{s}$, we have the equality

$$
\left.\frac{\partial^{s}}{\partial j_{1} \ldots \partial j_{s}} \operatorname{det}(1-\boldsymbol{j} \boldsymbol{A})^{-\alpha}\right|_{j=0}=\sum_{\sigma \in S_{s}} \rho_{\alpha}(\sigma) \prod_{i=1}^{s} \sum_{x_{i}=1}^{n} A_{x_{i}, x_{\sigma(i)}}^{(i)} \text {. }
$$

This result can be proved by induction over $s$. The right-hand side has a natural diagrammatic representation where each $i \in \mathbb{N}_{s}$ is a point and where an arrow is drawn from $i$ to $j$ whenever $\sigma(i)=j$. The sum in 109) is then the sum over all such diagrams in which each point $i \in \mathbb{N}_{s}$ has exactly one outgoing and one incoming arrow. The value of each diagram is a product of traces of the type $\operatorname{tr}\left(A^{(i)} A^{\sigma(i)} \cdots A^{\sigma^{p}(i)}\right)$, with $p$ being the smallest number in $\mathbb{N}_{0}$ such that $\sigma^{p+1}(i)=i$, weighted by its $\rho$ factor, which can be deduced from the number of connected sub-diagrams.

Let $q \geq 2$ and let $[\alpha] \equiv\left[\alpha_{0}, \alpha_{1}, \ldots, \alpha_{q-1}\right]$ be a list of $q$ directed bonds. The rule 109 can be applied to the expression 1077 for the generating function, and, making use of (76), one easily gets

$$
C_{[\alpha]}=\lim _{\epsilon \rightarrow 0} \frac{(2 \epsilon)^{q-1}}{2 B(q-1) !} \delta \xi_{[\alpha]}
$$


where $C_{[\alpha]}$ is the autocorrelation function defined in (42), and

$$
\left.\delta \xi_{[\alpha]} \equiv\left(\prod_{s=0}^{q-1} \frac{\partial}{\partial j_{s}}\right) \xi_{[\alpha]}\right|_{j=0} .
$$

The denominator $(q-1)$ ! in (110) comes from the number of diagrams arising when the rule (109) is applied to the $q-1$ retarded derivatives on $\xi_{[\alpha]}(\boldsymbol{j})$. By $[76)$, these diagrams all yield the same contribution.

It is not difficult to check that the generating functions have the following property. For all $j_{a}$ and $\boldsymbol{j}_{\boldsymbol{r}}=\left(j_{1}, \ldots, j_{q-1}\right)$ in a sufficiently small neighborhood of the origin,

$$
\xi_{[\alpha]}\left(j_{a}, \mathbf{0}\right)=\xi_{[\alpha]}\left(0, j_{r}\right)=1 .
$$

For $\sigma \in S_{q}$ and $[\alpha] \equiv\left[\alpha_{0}, \alpha_{1}, \ldots, \alpha_{q-1}\right]$, one can introduce a function $\xi_{[\alpha]}^{\sigma}(j)$ by the formula (106) using the matrices $E^{\alpha_{j}, \alpha_{\sigma(j)}}$ in place of $E^{\alpha_{j}, \alpha_{j}}$ in the source supermatrices, and (110) then serves as a definition for $C_{[\alpha]}^{\sigma}$. The function $\xi_{[\alpha]}^{\sigma}(\boldsymbol{j})$ also satisfies the property (112), and the identities (76) and (109) ensure that $C_{[\alpha]}^{\sigma}=C_{[\alpha]}$ for any $\sigma \in S_{q}$. In what follows, the arbitrary choice for $\sigma \in S_{q}$ in $\xi_{[\alpha]}^{\sigma}(j)$ will be called the choice of convention. These different but equivalent expressions must not be confused with the equivalent sums over orientated walks which are the object of Subsection 3.4 Any convention $\sigma \in S_{q}$ for the generating function involves $(q-1)$ ! equivalent sums over orientated walks. However, in the case $q=2$, the permutations $\sigma=\mathrm{id}$ and $\sigma=\left(\begin{array}{ll}0 & 1\end{array}\right)$, which are referred to as parallel and crossed conventions in the sequel, do correspond to the sums (83) and 85) respectively.

We started this chapter with the convention to choose $\sigma \in S_{q}$ to be the identity and we will use this convention in most of the following calculations. This convention is not only singled out by simplicity; it results in a generating function 106 that is explicitly gauge invariant while in other choices the gauge invariance is only restored in the limit (110). It also reduces the complexity of some calculations because the matrices $J_{r}\left(\boldsymbol{j}_{r}\right)$ and $J_{a}\left(j_{a}\right)$ for the source terms (103) are diagonal matrices. While each convention yields a different but exactly equivalent expression approximation schemes may break the exact identity. This is not worrying as long as the difference is in sub-leading order. For time-reversal invariant graphs the generating function (106) is usually not explicitly invariant when one replaces any directed bond by its reversed partner for $q \geq 3$. The invariance is only revealed once the derivative in (110) is taken (the limit $\epsilon \rightarrow 0$ is not required).

\subsection{Diagonal Approximation}

Before working further on the generating function with the supersymmetry method we introduce in this subsection similar generating functions and develop a corresponding trace formula. The diagonal approximation to this new type of generating functions turns out to behave very differently from the oriented walk representations previously discussed in the subsections 3.4 and 3.5 .

The definition (106) of the generating functions, and the fundamental formula (110) can easily be generalized to any correlation function (37) with $p=q$. Moreover, these 
correlation functions can also be written in terms of logarithmic derivatives with some analogy to (110). We will focus on the case $p=q=2$ for which the general correlation function can be written as

$$
\left\langle a_{\alpha_{1}}^{*} a_{\alpha_{1}^{\prime}}^{*} a_{\alpha_{2}} a_{\alpha_{2}^{\prime}}\right\rangle=\lim _{\epsilon \rightarrow 0} \frac{\epsilon}{B} \delta \Xi_{\left[\alpha_{1}, \alpha_{1}^{\prime} ; \alpha_{2}, \alpha_{2}^{\prime}\right]}
$$

where

$$
\begin{aligned}
\Xi_{\left[\alpha_{1}, \alpha_{1}^{\prime} ; \alpha_{2}, \alpha_{2}^{\prime}\right]}\left(j_{a}, j_{r}\right) & \equiv\left\langle\log \operatorname{det}\left(1-\tilde{J}_{r}\left(j_{r}\right) U_{\epsilon}(k)\right) \log \operatorname{det}\left(1-\tilde{J}_{a}\left(j_{a}\right) U_{\epsilon}^{\dagger}(k)\right)\right\rangle_{k} \\
& =\left\langle\log \operatorname{det}\left(1-\tilde{J}_{r}\left(j_{r}\right) U_{\epsilon}(k)\right) \log \operatorname{det}\left(1-\tilde{J}_{a}\left(j_{a}\right)^{T} U_{\epsilon}(k)\right)^{*}\right\rangle_{k},
\end{aligned}
$$

the source terms are given by

$$
\tilde{J}_{r}\left(j_{r}\right)=1+j_{r} E^{\alpha_{1}, \alpha_{2}} \quad \text { and } \quad \tilde{J}_{a}\left(j_{a}\right)=1+j_{a} E^{\alpha_{1}^{\prime}, \alpha_{2}^{\prime}},
$$

and $\left.\delta \Xi \equiv \frac{\partial^{2}}{\partial j_{r} \partial j_{a}} \Xi\left(j_{a}, j_{r}\right)\right|_{j_{a}=j_{r}=0}$. The intensity correlation matrix can be obtained in two different ways

$$
\begin{aligned}
C_{\alpha \alpha^{\prime}} & =\lim _{\epsilon \rightarrow 0} \frac{\epsilon}{B} \delta \Xi_{\left[\alpha, \alpha^{\prime} ; \alpha, \alpha^{\prime}\right]} \\
& =\lim _{\epsilon \rightarrow 0} \frac{\epsilon}{B} \delta \Xi_{\left[\alpha, \alpha^{\prime} ; \alpha^{\prime}, \alpha\right]}
\end{aligned}
$$

referred as parallel and crossed conventions, respectively. In the orthogonal class one has a third representation

$$
C_{\alpha \alpha^{\prime}}=\lim _{\epsilon \rightarrow 0} \frac{\epsilon}{B} \delta \Xi_{\left[\alpha, \hat{\alpha} ; \alpha^{\prime}, \hat{\alpha}^{\prime}\right]}
$$

called time-reversed crossed convention.

The formula $\log$ det $=\operatorname{tr} \log$ enables us to write the new generating function (114) in terms of generalized periodic orbits on the graph. Indeed, expanding the logarithms and performing the spectral average yields the trace formula

$$
\Xi_{\left[\alpha_{1}, \alpha_{1}^{\prime} ; \alpha_{2}, \alpha_{2}^{\prime}\right]}\left(j_{a}, j_{r}\right)=\sum_{p \in P_{\alpha_{1} \alpha_{2}}} \sum_{p^{\prime} \in P_{\alpha_{2}^{\prime} \alpha_{1}^{\prime}}} \sum_{\rho, \rho^{\prime}=0}^{\infty} \frac{1}{\rho \rho^{\prime}} A_{r, p}\left(j_{r}\right)^{\rho}\left(A_{a, p^{\prime}}\left(j_{a}\right)^{*}\right)^{\rho^{\prime}} \delta_{\rho l_{p}, \rho^{\prime} l_{p^{\prime}}}
$$

where the retarded and advanced modified stability amplitudes $A_{r, p}\left(j_{r}\right)$ and $A_{a, p}\left(j_{a}\right)$ of the generalized periodic orbit $p=\overline{\beta_{1} \beta_{2} \ldots \beta_{|p|}}$ are defined by

$$
A_{r, p}\left(j_{r}\right) \equiv \prod_{i=1}^{|p|}\left[\tilde{J}_{r}\left(j_{r}\right) S_{\epsilon}\right]_{\beta_{i+1} \beta_{i}} \quad \text { and } \quad A_{a, p}\left(j_{a}\right) \equiv \prod_{i=1}^{|p|}\left[\tilde{J}_{a}\left(j_{a}\right)^{T} S_{\epsilon}\right]_{\beta_{i+1} \beta_{i}} .
$$

The periodic orbits $p$ and $p^{\prime}$ in (119) are all primitive but can be of a slightly more general type than the primitive periodic orbits considered in Subsection 3.5 Indeed, the retarded source term in 115 introduces the possibility to jump $\alpha_{2} \curvearrowright \alpha_{1}$, and similarly the advanced source term introduces the possibility to jump $\alpha_{1}^{\prime} \curvearrowright \alpha_{2}^{\prime}$. The set $P_{\alpha_{1} \alpha_{2}}$ in 119 then contains all the primitive periodic orbits that are compatible with the topology of the graph with an additional bridge $\alpha_{2} \curvearrowright \alpha_{1}$ at the center of these 
directed bonds. Note that the two sets $P_{\alpha_{1} \alpha_{2}}$ and $P_{\alpha_{2}^{\prime} \alpha_{1}^{\prime}}$ of generalized periodic orbits need not be identical. In the parallel convention for $C_{\alpha \alpha^{\prime}}$, the source terms are diagonal matrices, and $P_{\alpha \alpha} \equiv P$ reduces to the set of standard primitive periodic orbits, which only respect the topology of the graph. The length of a generalized periodic orbit is just the sum of all bond lengths along the periodic orbit, where every jump $\alpha_{2} \curvearrowright \alpha_{1}$ contributes $\frac{1}{2}\left(L_{\alpha_{1}}+L_{\alpha_{2}}\right)$. In the trace formula (119), only pairs of primitive orbits contribute such that a repetition of one orbit has the same length as a repetition of the other.

The diagonal approximation to the trace formula (119) reduces the sum over pairs of primitive orbits to either equal orbits $p^{\prime}=p$, or time-reversed orbits $p^{\prime}=\hat{p}$. In both cases, the factor $\delta_{\rho l_{p}, \rho^{\prime} l_{p^{\prime}}}$ enforces $\rho=\rho^{\prime}$. Note that $p$ has to be a periodic orbit in the intersection $p \in P_{\alpha_{1} \alpha_{2}} \cap P_{\alpha_{2}^{\prime} \alpha_{1}^{\prime}}$ to contribute to the diagonal approximation.

The remaining sum over periodic orbits can be resummed

$$
\begin{aligned}
\Xi_{\left[\alpha_{1}, \alpha_{1}^{\prime} ; \alpha_{2}, \alpha_{2}^{\prime}\right]}^{\mathrm{diag}}\left(j_{a}, j_{r}\right) & =\Xi_{\left[\alpha_{1}, \alpha_{1}^{\prime} ; \alpha_{2}, \alpha_{2}^{\prime}\right]}^{\mathrm{diag},\left(j_{a}, j_{r}\right)} & +\Xi_{\left[\alpha_{1}, \alpha_{1}^{\prime} ; \alpha_{2}, \alpha_{2}^{\prime}\right]}^{\mathrm{diag},}\left(j_{a}, j_{r}\right) & +C \\
& =-\log \operatorname{det}\left(1-M^{D}\left(j_{a}, j_{r}\right)\right) & -\log \operatorname{det}\left(1-M^{C}\left(j_{a}, j_{r}\right)\right) & +C
\end{aligned}
$$

where $M^{D}\left(j_{a}, j_{r}\right)$ and $M^{C}\left(j_{a}, j_{r}\right)$ are modifications of the classical map (32). They describe diffuson and cooperon propagations, which originate from pairs of periodic orbits with $p^{\prime}=p$ and $p^{\prime}=\hat{p}$, and are given by

$$
\begin{aligned}
M^{D}\left(j_{a}, j_{r}\right)_{\beta_{1} \beta_{2}} \equiv & \sum_{\beta^{\prime}} J_{r}\left(j_{r}\right)_{\beta_{1} \beta^{\prime}} J_{a}\left(j_{a}\right)_{\beta^{\prime} \beta_{1}}\left|S_{\epsilon, \beta^{\prime} \beta_{2}}\right|^{2} \\
= & \left(1+j_{r} \delta_{\beta_{1} \alpha_{1}} \delta_{\alpha_{1} \alpha_{2}}+j_{a} \delta_{\beta_{1} \alpha_{2}^{\prime}} \delta_{\alpha_{1}^{\prime} \alpha_{2}^{\prime}}\right)\left|S_{\epsilon, \beta_{1} \beta_{2}}\right|^{2}+ \\
& \left.j_{r} j_{a} \delta_{\beta_{1} \alpha_{1}} \delta_{\alpha_{1} \alpha_{2}^{\prime}} \delta_{\alpha_{1}^{\prime} \alpha_{2}} S_{\epsilon, \alpha_{2} \beta_{2}}\right|^{2} \\
M^{C}\left(j_{a}, j_{r}\right)_{\beta_{1} \beta_{2}} \equiv & \sum_{\beta^{\prime}} J_{r}\left(j_{r}\right)_{\beta_{1} \beta^{\prime}} J_{a}\left(j_{a}\right)_{\beta^{\prime} \beta_{1}}^{\mathcal{T}} S_{\epsilon, \beta^{\prime} \beta_{2}}\left(S_{\epsilon, \beta^{\prime} \beta_{2}}^{\mathcal{T}}\right)^{*} \\
= & \left(1+j_{r} \delta_{\beta_{1} \alpha_{1}} \delta_{\alpha_{1} \alpha_{2}}+j_{a} \delta_{\beta_{1} \hat{\alpha}_{1}^{\prime}} \delta_{\alpha_{1}^{\prime} \alpha_{\alpha}^{\prime}}\right) S_{\epsilon, \beta_{1} \beta_{2}}\left(S_{\epsilon, \hat{\beta}_{2} \hat{\beta}_{1}}\right)^{*}+ \\
& j_{r} j_{a} \delta_{\beta_{1} \alpha_{1}} \delta_{\alpha_{1} \hat{\alpha}_{1}^{\prime}} \delta_{\alpha_{2} \hat{\alpha}_{2}^{\prime}} S_{\epsilon, \alpha_{2} \beta_{2}}\left(S_{\epsilon, \hat{\beta}_{2} \hat{\alpha}_{2}}\right)^{*} .
\end{aligned}
$$

The term $C$ in (121) contains corrections for repetitions and self-retracing orbits $(p=\hat{p})$ which can be shown not to contribute to our final result and will be omitted henceforth.

Recall that the classical map is defined by $M_{\beta_{1} \beta_{2}}=\left|S_{\beta_{1} \beta_{2}}\right|^{2}$, so that $M^{D}(0,0)=M_{\epsilon} \equiv$ $e^{-2 \epsilon} M$. For time-reversal invariant systems, the products of scattering matrices in (123) reduces to $S_{\epsilon, \beta_{1} \beta_{2}}\left(S_{\epsilon, \hat{\beta}_{2} \hat{\beta}_{1}}\right)^{*}=\left|S_{\epsilon, \beta_{1} \beta_{2}}\right|^{2}=M_{\epsilon, \beta_{1} \beta_{2}}$, so that $\lim _{\epsilon \rightarrow 0} M^{C}(0,0)=M$. If time reversal symmetry is broken $M^{C}(0,0)$ does not reduce to $M$ and it does not describe a Markof process on the graph. We will see that the cooperon term only contributes to the diagonal approximation formula if time-reversal symmetry holds. 
The derivatives with respect to $j_{r}$ and $j_{a}$ can now be taken and yield

$$
\begin{aligned}
\delta \Xi_{\left[\alpha_{1}, \alpha_{1}^{\prime} ; \alpha_{2}, \alpha_{2}^{\prime}\right]}^{\operatorname{diag}, D}= & {\left[\operatorname{tr}\left(\frac{1}{1-M_{\epsilon}} \frac{\partial^{2} M^{D}}{\partial j_{+} \partial j_{-}}\right)+\operatorname{tr}\left(\frac{1}{1-M_{\epsilon}} \frac{\partial M^{D}}{\partial j_{+}} \frac{1}{1-M_{\epsilon}} \frac{\partial M^{D}}{\partial j_{-}}\right)\right]_{j_{a}=j_{r}=0} } \\
= & \delta_{\alpha_{1} \alpha_{2}^{\prime}} \delta_{\alpha_{1}^{\prime} \alpha_{2}}\left(\frac{M_{\epsilon}}{1-M_{\epsilon}}\right)_{\alpha_{2} \alpha_{1}}+ \\
& \delta_{\alpha_{1} \alpha_{2}} \delta_{\alpha_{1}^{\prime} \alpha_{2}^{\prime}}\left(\frac{M_{\epsilon}}{1-M_{\epsilon}}\right)_{\alpha_{1} \alpha_{1}^{\prime}}\left(\frac{M_{\epsilon}}{1-M_{\epsilon}}\right)_{\alpha_{1}^{\prime} \alpha_{1}}
\end{aligned}
$$

for the diffuson generating function, and

$$
\begin{aligned}
\delta \Xi_{\left[\alpha_{1}, \alpha_{1}^{\prime} ; \alpha_{2}, \alpha_{2}^{\prime}\right]}^{\mathrm{diag}, C}= & \delta_{\alpha_{1} \hat{\alpha}_{1}^{\prime}} \delta_{\alpha_{2} \hat{\alpha}_{2}^{\prime}}\left(\frac{M^{C}(0,0)}{1-M^{C}(0,0)}\right)_{\alpha_{2} \alpha_{1}}+ \\
& \delta_{\alpha_{1} \alpha_{2}} \delta_{\alpha_{1}^{\prime} \alpha_{2}^{\prime}}\left(\frac{M^{C}(0,0)}{1-M^{C}(0,0)}\right)_{\alpha_{1} \hat{\alpha}_{1}^{\prime}}\left(\frac{M^{C}(0,0)}{1-M^{C}(0,0)}\right)_{\alpha_{1}^{\prime} \hat{\alpha}_{1}}
\end{aligned}
$$

for the cooperon generating function. For broken time-reversal invariance the classical cooperon map $M^{C}(0,0)$ has no unit eigenvalues in the limit $\epsilon \rightarrow 0$, and hence, (125) identically vanishes in that limit. By contrast, in time-reversal invariant systems $M^{C}(0,0)=M_{\epsilon}$ and the cooperon generating function does contribute.

Finally, only terms in (124) and (125) that are singular as $\epsilon \rightarrow 0$ contribute to the correlation function (113). In order to isolate these terms, one makes use of the decomposition (35) of classical orbits as the sum of a uniform component $|1\rangle\langle 1|$ and a massive part $R$. This yields

$$
\begin{aligned}
\frac{\epsilon}{B} \delta \Xi_{\left[\alpha_{1}, \alpha_{1}^{\prime} ; \alpha_{2}, \alpha_{2}^{\prime}\right]}^{\mathrm{diag}}= & \frac{\kappa(1-2 \epsilon)}{16 B^{3} \epsilon} \delta_{\alpha_{1} \alpha_{2}} \delta_{\alpha_{1}^{\prime} \alpha_{2}^{\prime}}+\frac{1}{4 B^{2}}\left(\delta_{\alpha_{1} \alpha_{2}^{\prime}} \delta_{\alpha_{1}^{\prime} \alpha_{2}}+(\kappa-1) \delta_{\alpha_{1} \hat{\alpha}_{1}^{\prime}} \delta_{\alpha_{2} \hat{\alpha}_{2}^{\prime}}\right)+ \\
& +\frac{1}{4 B^{2}} \delta_{\alpha_{1} \alpha_{2}} \delta_{\alpha_{1}^{\prime} \alpha_{2}^{\prime}}\left(\left[R_{\alpha_{1} \alpha_{1}^{\prime}}+R_{\alpha_{1}^{\prime} \alpha_{1}}\right]+(\kappa-1)\left[R_{\alpha_{1} \hat{\alpha}_{1}^{\prime}}+R_{\hat{\alpha}_{1}^{\prime} \alpha_{1}}\right]\right)+O(\epsilon)
\end{aligned}
$$

After dropping terms that are $O(\epsilon)$, 126 may be expected to provide an approximation to the generating function (113). However, its first term diverges like $\epsilon^{-1}$ as $\epsilon \rightarrow 0$. At first sight, this seems to make periodic-orbit analysis using the trace formula 119 for the generating function much less useful than the previous trace formulae from Section 3.4 which behave nicely in the diagonal approximation. On the other hand, the same divergence also occurs in the analysis of spectral correlations, which become singular in the diagonal approximation at small energy differences. Indeed, one may obtain the corresponding trace formula for the spectral two point correlation function on a graph $R_{2}(s)$ by replacing the source terms $\tilde{J}_{a}\left(j_{a}\right)$ and $\tilde{J}_{r}\left(j_{r}\right)$ appropriately. In this context, a supersymmetry method developed in [22, 23], which will be adapted to our purposes in what follows, cures the divergence. One may also try to add off-diagonal terms in the trace formula in a systematic way but we will not pursue this here.

Note that the Kronecker symbols in the expression (126) force the correlation function $\left\langle a_{\alpha_{1}}^{*} a_{\alpha_{1}^{\prime}}^{*} a_{\alpha_{2}} a_{\alpha_{2}^{\prime}}\right\rangle$ to vanish for all combinations that are not invariant under all local 
gauge transformations allowed by the unitary or orthogonal symmetry class. The nonvanishing combinations are then equivalent to the three different conventions (116), (117) and (118) for expressing the intensity correlation matrix. These three conventions lead however to different formulae.

For any of the three conventions (116), 117) and 118), if one replaces $\epsilon \mapsto \frac{\kappa}{4 B}$, the first line in (126) reproduces the prediction of the Gaussian Random Wave Model up to corrections that are $O\left(B^{-3}\right)$. The second line in 126 then gives a correction in terms of system dependent massive modes. This massive correction turns out to be different for the three conventions of expressing $C_{\alpha \alpha^{\prime}}$.

In the orthogonal class only the parallel convention 116 provides an approximated intensity correlation matrix that respects the identity $C_{\alpha \alpha^{\prime}}=C_{\alpha \hat{\alpha}^{\prime}}$ satisfied by the exact intensity correlation matrix. By contrast, if either the crossed convention (117) or the time-reversed crossed convention (118) is used, the massive terms in the approximated intensity correlation matrix explicitly violate this symmetry. The origin of this discrepancy is that, with the parallel convention (116), each of the two logarithms in the generating function $\Xi_{\left[\alpha, \alpha^{\prime} ; \alpha, \alpha^{\prime}\right]}\left(j_{a}, j_{r}\right)$ is invariant under time inversion, while in the crossed and time-reversed crossed conventions the symmetry is only restored after taking the derivatives and performing the limit $\epsilon \rightarrow 0$.

The observations above concerning time-reversal symmetry makes the parallel convention (116) a privileged choice when it comes to the diagonal approximation. This convention yields

$$
C_{\alpha \alpha^{\prime}}^{\mathrm{diag}, \|}=\frac{1}{4 B^{2}}\left(\frac{\kappa(1-2 \epsilon)}{4 B \epsilon}+\delta_{\alpha \alpha^{\prime}}+(\kappa-1) \delta_{\alpha \hat{\alpha}^{\prime}}+R_{\alpha \alpha^{\prime}}+R_{\alpha^{\prime} \alpha}+(\kappa-1)\left(R_{\alpha \hat{\alpha}^{\prime}}+R_{\hat{\alpha}^{\prime} \alpha}\right)\right)
$$

The three first terms are universal, and are equal to the prediction of the Gaussian Random Wave Model if $\epsilon$ is chosen finite and set equal to $\frac{K}{4 B}$ (which we cannot justify at this stage). The remaining three terms involve the matrix $R$ and they describe massive corrections to the universal result. In fact, these massive contributions may dominate the correlation functions and, as a consequence, the rate of convergence for quantum ergodicity, or they may destroy quantum ergodicity altogether. This point will be discussed further in Section 7

\subsection{Nonlinear Supersymmetric $\sigma$ Model}

The generating functions in 106 depend strongly on whether time-reversal symmetry is broken or conserved. Time inversion acts on supervectors $\psi$ in the Grassmann envelope $(X \oplus \mathcal{X})(\Lambda)$, defined from $\mathcal{X}=\mathcal{A} \otimes \mathbb{C}^{n}$ for some $n \in \mathbb{N}_{0}$ as in 102, and on supermatrices $A \in L(\mathcal{X} \mid \mathcal{X})$ as

$$
\mathcal{T} \psi=\sigma_{1}^{d} \psi^{*} \quad \text { and } \quad A^{\mathcal{T}}=\sigma_{1}^{d} A^{T} \sigma_{1}^{d} .
$$

In (128), $\sigma_{1}^{d}$ is the first Pauli matrix acting on the direction space $\mathcal{A}_{d}, \psi^{*}$ denotes the vector obtained from $\psi$ by taking the complex conjugates of each component, and $A^{T}$ is the transpose of $A$ defined as in [19] by the condition $\left(A \psi_{1}\right)^{T} \psi_{2}=\psi_{1}^{T} A^{T} \psi_{2}$ for all $\psi_{1}, \psi_{2}$ in $(\mathcal{X} \oplus \mathcal{X})(\Lambda)$. Here and henceforth, $\psi^{T}$ stands for the row vector obtained from 
the column vector $\psi \in(\mathcal{X} \oplus \mathcal{X})(\Lambda)$ by usual transposition. One can now introduce a 2-dimensional $\mathbb{C}$-linear space $T R$, the time-reversal space, and the mapping

$$
\psi \mapsto \Psi \equiv \frac{1}{\sqrt{2}}\left(\begin{array}{c}
\psi \\
\mathcal{T} \psi
\end{array}\right)_{T R}=\frac{1}{\sqrt{2}}\left(\begin{array}{c}
\psi \\
\sigma_{1}^{d} \psi^{*}
\end{array}\right)_{T R},
$$

from $(X \oplus \mathcal{X})(\Lambda)$ to $(X \otimes T R \oplus X \otimes T R)(\Lambda)$ called time-reversal doubling. We work with the convention $\chi^{* *}=-\chi$ for all $\chi \in \Lambda_{F}$ as in [19], and hence, the Hermitian conjugate of $\Psi$ in (129) reads

$$
\bar{\Psi}=\left(\psi^{\dagger}, \psi^{T} \sigma_{1}^{d} \sigma_{3}^{B F}\right),
$$

where $\psi^{\dagger}=\psi^{* T}$ is the Hermitian conjugate of $\psi$, and $\sigma_{3}^{B F}$ stands for the third Pauli matrix acting on the Bose-Fermi space. Similarly, the time-reversal doubling of a supermatrix $A \in L(\mathcal{X} \mid \mathcal{X})$ is defined by

$$
A \mapsto \mathcal{A} \equiv\left(\begin{array}{cc}
A & 0 \\
0 & A^{\mathcal{T}}
\end{array}\right)_{T R}=\left(\begin{array}{cc}
A & 0 \\
0 & \sigma_{1}^{d} A^{T} \sigma_{1}^{d}
\end{array}\right)_{T R},
$$

and is an element of $L(X \otimes T R \mid X \otimes T R)$. In (129), 1131) and in what follows, an index $T R$ added to a supermatrix means that this supermatrix is explicitly written in the $T R$ space, and the same notational trick is used for any other space. The components in time-reversal space will be indexed by $t \in\{\uparrow, \downarrow\}$. The definitions above call for a notion of generalized transposition $\mathcal{A}^{\tau}$ of $\mathcal{A} \in L(\mathcal{X} \otimes T R \mid \mathcal{X} \otimes T R)$, which is defined as in [23] by

$$
\mathcal{A}^{\tau} \equiv \tau \mathcal{A}^{T} \tau^{-1}, \quad \text { where } \quad \tau \equiv \sigma_{1}^{d}\left(\begin{array}{cc}
0 & \sigma_{3}^{B F} \\
\mathbb{1}_{B F} & 0
\end{array}\right)_{T R} .
$$

This definition implies that the equality $\bar{\Psi}_{1} \mathcal{A} \Psi_{2}=\bar{\Psi}_{2} \mathcal{A}^{\tau} \Psi_{1}$ holds for any couple of

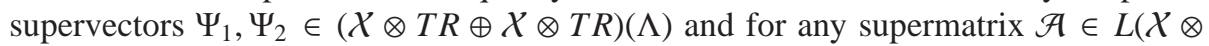
$T R \mid X \otimes T R)$. It follows that $\left(\mathcal{A}^{\tau}\right)^{\tau}=\mathcal{A}$ and $(\mathcal{A B})^{\tau}=\mathcal{B}^{\tau} \mathcal{A}^{\tau}$ for any such supermatrices. Moreover, using the property $\left(A^{T}\right)^{T}=\sigma_{3}^{B F} A \sigma_{3}^{B F}$ of the transposition in $L(\mathcal{X} \mid \mathcal{X})$, it is easy to check that a supermatrix $\mathcal{A}$ obtained from some $A \in L(X \mid X)$ by time-reversal doubling (131) is invariant under generalized transposition.

Now, the generating functions can be written

$$
\xi_{[\alpha]}(j)=\operatorname{sdet}^{-1} J_{r} J_{a}\left\langle\operatorname{sdet}^{-\frac{1}{2}}\left(\mathcal{J}_{r}^{-1}-\mathcal{U}_{\epsilon}(k)\right)\left(\mathcal{J}_{a}^{-1}-\mathcal{U}_{\epsilon}^{\dagger}(k)\right)\right\rangle_{k},
$$

where $\mathcal{J}_{r / a}$ and $\mathcal{U}_{\epsilon}(k)$ are the time-reversal doubles of $J_{r / a}$ and $U_{\epsilon}(k)$. Following the scheme developed in [22] and [23], the generating functions (133) can be represented in terms of a nonlinear supersymmetric $\sigma$ model.

First, it is convenient to make use of the equality

$$
\operatorname{sdet}\left(\begin{array}{cc}
A & B \\
C & D
\end{array}\right)=\operatorname{sdet}(A D) \operatorname{sdet}\left(1-A^{-1} B D^{-1} C\right)
$$

that holds for any square supermatrices $A, B, C$ and $D$ of the same size, and write the retarded and advanced superdeterminants in (133) as

$$
\operatorname{sdet}^{-1 / 2}\left(\mathcal{J}_{r}^{-1}-\mathcal{U}_{\epsilon}\right)=\operatorname{sdet}^{-1 / 2}\left(\begin{array}{cc}
1 & \sqrt{\mathcal{S}_{\epsilon}} T \\
T \sqrt{\mathcal{S}_{\epsilon}} & \mathcal{J}_{r}^{-1}
\end{array}\right)
$$


and

$$
\operatorname{sdet}^{-1 / 2}\left(\mathcal{J}_{a}^{-1}-\mathcal{U}_{\epsilon}^{\dagger}\right)=\operatorname{sdet}^{-1 / 2}\left(\begin{array}{cc}
1 & \sqrt{\mathcal{S}_{\epsilon}^{\dagger}} T^{\dagger} \\
T^{\dagger} \sqrt{\mathcal{S}_{\epsilon}^{\dagger}} & \mathcal{J}_{a}^{-1}
\end{array}\right) .
$$

In these expressions, $\mathcal{S}_{\epsilon}$ is the time-reversal double of $S_{\epsilon}$, and the square root of a matrix is defined by keeping the same eigenvectors and by taking the square roots of the eigenvalues fixing the half-line singularity of the logarithm to $(-\infty, 0]$. It is not difficult to check that this definition of the square root leads to the natural properties $\sqrt{A} \sqrt{A}=A$ and $\sqrt{A^{\dagger}}=\sqrt{A^{\dagger}}$. These two properties have been used in order to obtain (135) and (136). Besides, if $\mathcal{A}$ is the time-reversal double of $A$, then $\sqrt{\mathcal{A}}$ is the time-reversal double of $\sqrt{A}$. The 2-dimensional structure introduced in the right-hand sides of (135) and (136) is referred to as the auxiliary space $X$, and the components with respect to the basis of $X$ used in these two matrix expressions will be indexed by $x \in\{1,2\}$. The formulae (133), (135) and (136) now enable us to express the generating functions as the Gaussian superintegrals

$$
\xi_{[\alpha]}(\boldsymbol{j})=\operatorname{sdet}^{-1} J_{r} J_{a} \cdot \int d \psi\left\langle e^{-S[\Psi]}\right\rangle_{k},
$$

where $d \psi=d \psi_{r} d \psi_{a}, d \psi_{r}$ and $d \psi_{a}$ being two Berezin measures [5] on $(\mathcal{A} \oplus \mathcal{A})(\Lambda), \Psi$ is the time-reversal double of $\psi$, and

$$
\begin{aligned}
S[\Psi] \equiv & \left(\bar{\Psi}_{r 1} \bar{\Psi}_{r 2}\right)\left(\begin{array}{cc}
1 & \sqrt{\mathcal{S}_{\epsilon}} T \\
T \sqrt{\mathcal{S}_{\epsilon}} & \mathcal{J}_{r}^{-1}
\end{array}\right)\left(\begin{array}{l}
\Psi_{r 1} \\
\Psi_{r 2}
\end{array}\right) \\
& +\left(\bar{\Psi}_{a 1} \bar{\Psi}_{a 2}\right)\left(\begin{array}{cc}
1 & \sqrt{\mathcal{S}}_{\epsilon}^{\dagger} T^{\dagger} \\
T^{\dagger}{\sqrt{\mathcal{S}_{\epsilon}}}^{\dagger} & \mathcal{J}_{a}^{-1}
\end{array}\right)\left(\begin{array}{l}
\Psi_{a 1} \\
\Psi_{a 2}
\end{array}\right) .
\end{aligned}
$$

The indices $r$ and $a$ of $\psi$ and $\Psi$ refer to the retarded and advanced components of these supervectors used to write (135) and (136) as Gaussian superintegrals respectively.

In the quadratic form (138), the off-diagonal couplings depend on $k$ through the variables $\left(k L_{1}, \ldots, k L_{B}\right)$ in the propagation matrix $T$. Using the fact that $T$ and $\sqrt{\mathcal{S}_{\epsilon}}$ are invariant under generalized transposition, these off-diagonal terms can be written

$$
S_{\mathrm{cf}}[\Psi]=2 \bar{\Psi}_{r 1} \sqrt{\mathcal{S}_{\epsilon}} T \Psi_{r 2}+2 \bar{\Psi}_{a 2} T^{\dagger} \sqrt{\mathcal{S}_{\epsilon}^{\dagger}} \Psi_{a 1} .
$$

Since the bond lengths are assumed incommensurate the invariant measure of the automorphism $k \mapsto\left(k L_{1}, \ldots, k L_{B}\right)(\bmod 2 \pi)$ on the $B$-torus is merely the product of $B$ Haar measures on the circle [4]. Hence,

$$
\left\langle e^{-S_{\mathrm{cf}}[\Psi]}\right\rangle_{k}=\prod_{b=1}^{B} \int_{0}^{2 \pi} \frac{d \varphi_{b}}{2 \pi} e^{-S_{\mathrm{cf}}^{b}\left[\Psi_{b} ; \varphi_{b}\right]},
$$

where

$$
S_{\mathrm{cf}}^{b}\left[\Psi_{b} ; \varphi_{b}\right] \equiv 2 \sum_{d= \pm}\left[\left(\bar{\Psi}_{r 1} \sqrt{\mathcal{S}_{\epsilon}}\right)_{b d} e^{i \varphi_{b}} \Psi_{r 2 ; b d}+\bar{\Psi}_{a 2 ; b d} e^{-i \varphi_{b}}\left(\sqrt{\mathcal{S}_{\epsilon}^{\dagger}} \Psi_{a 1}\right)_{b d}\right] .
$$

Then, the color-flavor transformation [51] can be applied separately to each integral in the right-hand side of $(140)$. This procedure introduces supermatrix variables $Z_{b}$ and 
$\tilde{Z}_{b}$, which lie in $L\left(\mathcal{A}_{d} \otimes T R \mid \mathcal{A}_{d} \otimes T R\right)$, and yields

$$
\left\langle e^{-S_{\mathrm{cf}}[\Psi]}\right\rangle_{k}=\prod_{b=1}^{B} \int d\left(Z_{b}, \tilde{Z}_{b}\right) \operatorname{sdet}\left(1-Z_{b} \tilde{Z}_{b}\right) e^{-S_{\mathrm{cf}}^{b}\left[\Psi_{b} ; Z_{b}, \tilde{Z}_{b}\right]},
$$

with

$$
\begin{aligned}
S_{\mathrm{cf}}^{b}\left[\Psi_{b} ; Z_{b}, \tilde{Z}_{b}\right]= & 2 \sum_{d= \pm}\left(\bar{\Psi}_{r 1} \sqrt{\mathcal{S}_{\epsilon}}\right)_{b d} Z_{b ; d d^{\prime}}\left(\sqrt{\mathcal{S}_{\epsilon}^{\dagger}} \Psi_{a 1}\right)_{b d^{\prime}} \\
& +2 \sum_{d= \pm} \bar{\Psi}_{a 2 ; b d} \tilde{Z}_{b, d d^{\prime}} \Psi_{r 2 ; b d}
\end{aligned}
$$

Basically, the retarded and advanced components of $\Psi$, which are uncoupled in (141) become coupled in (143), and conversely, the components in auxiliary space, which are mixed in 141), are diagonalized by the color-flavor transformation. The reason for resorting to this transformation is to get an action with saddle-points, which is not the case in (141). The integration in (142) must be performed over the set of supermatrices $\left(Z_{b}, \tilde{Z}_{b}\right)$ satisfying the conditions

$$
\tilde{Z}_{B B}=Z_{B B}^{\dagger}, \quad \tilde{Z}_{F F}=-Z_{F F}^{\dagger},
$$

and such that the eigenvalues of the positive hermitian matrix $Z_{B B}^{\dagger} Z_{B B}$ are less than unity. The measure $d\left(Z_{b}, \tilde{Z}_{b}\right)$ is then the Berezin measure over this set.

In order to simplify the notation, one can introduce the new supermatrix fields

$$
Z=\bigoplus_{b=1}^{B} Z_{b} \quad \text { and } \quad \tilde{Z}=\bigoplus_{b=1}^{B} \tilde{Z}_{b},
$$

which belong to $L(\mathcal{A} \otimes T R \mid \mathcal{A} \otimes T R)$. These supermatrices still satisfy the color-flavor requirements (144). From (143) and the diagonal terms of (138), one gets the new quadratic form

$$
\begin{aligned}
& S[\Psi ; Z, \tilde{Z}]=\left(\begin{array}{cc}
\bar{\Psi}_{r 1} \bar{\Psi}_{a 1}
\end{array}\right)\left(\begin{array}{cc}
1 & \sqrt{\mathcal{S}_{\epsilon}} Z \sqrt{\mathcal{S}_{\epsilon}^{\dagger}} \\
\sqrt{\mathcal{S}_{\epsilon}} Z^{\tau} \sqrt{\mathcal{S}_{\epsilon}} & 1
\end{array}\right)\left(\begin{array}{l}
\Psi_{r 1} \\
\Psi_{a 1}
\end{array}\right) \\
& +\left(\bar{\Psi}_{r 2} \bar{\Psi}_{a 2}\right)\left(\begin{array}{cc}
\mathcal{J}_{r}^{-1} & \tilde{Z}^{\tau} \\
\tilde{Z} & \mathcal{J}_{a}^{-1}
\end{array}\right)\left(\begin{array}{c}
\Psi_{r 2} \\
\Psi_{a 2}
\end{array}\right) .
\end{aligned}
$$

The integral over $\psi$ in (137) remains Gaussian after the color-flavor transformation, and, from the explicit formula (146), the generating functions become

$$
\begin{gathered}
\xi_{[\alpha]}(j)=\operatorname{sdet}^{-1} J_{r} J_{a} \int d(Z, \tilde{Z}) \operatorname{sdet}(1-Z \tilde{Z}) \operatorname{sdet}^{-1 / 2}\left(\begin{array}{cc}
\mathcal{J}_{r}^{-1} & \tilde{Z}^{\tau} \\
\tilde{Z} & \mathcal{J}_{a}^{-1}
\end{array}\right) \\
\operatorname{sdet}^{-1 / 2}\left(\begin{array}{cc}
1 & \sqrt{\mathcal{S}_{\epsilon}} Z \sqrt{\mathcal{S}_{\epsilon}^{\dagger}} \\
\sqrt{\mathcal{S}_{\epsilon}^{\dagger}} Z^{\tau} \sqrt{\mathcal{S}_{\epsilon}} & 1
\end{array}\right) .
\end{gathered}
$$

The first superdeterminant in the integrand comes from the $B$ superdeterminant factors introduced in 142). Making use of the rule (134) once again and resorting to the well-known formula sdet $=\exp$ str $\log$ enables us to write

$$
\xi_{[\alpha]}(\boldsymbol{j})=\int d(Z, \tilde{Z}) e^{-S[Z, \tilde{Z}]},
$$


where the function $S[Z, \tilde{Z}]$, called the action, or the exact action in order to distinguish between $S[Z, \tilde{Z}]$ and its subsequent approximations, is defined by

$$
\begin{aligned}
S[Z, \tilde{Z}]= & -\operatorname{str} \log (1-Z \tilde{Z})+\frac{1}{2} \operatorname{str} \log \left(1-Z \mathcal{S}_{\epsilon}^{\dagger} Z^{\tau} \mathcal{S}_{\epsilon}\right) \\
& +\frac{1}{2} \operatorname{str} \log \left(1-\mathcal{J}_{r} \tilde{Z}^{\tau} \mathcal{J}_{a} \tilde{Z}\right) .
\end{aligned}
$$

Notice that, if the sources $\boldsymbol{j}_{\boldsymbol{r}}$ and $j_{a}$ are set to zero, the resulting source-free action $S_{0}[Z, \tilde{Z}]$ is precisely the one obtained in [22] and [23] for the generating function of the spectral two-point correlation function.

The different conventions $\sigma \in S_{q}$ for the generating functions discussed at the end of Subsection 4.1 can also be written in terms of the nonlinear supersymmetric $\sigma$ model (148). Indeed, in order to get $\xi_{[\alpha]}^{\sigma}(j)$, it suffices to replace $J_{r}$ and $J_{a}$ with $J_{r}^{\sigma}$ and $J_{a}^{\sigma}$ in the exact action, where these two new source supermatrices are defined as in 103) and (104) using the matrices $E^{\alpha_{j}, \alpha_{\sigma(j)}}$ instead of $E^{\alpha_{j}, \alpha_{j}}$ for all $0 \leq j \leq q-1$.

\section{Mean Field Theory}

\subsection{The Zero Mode}

The first step of our approximation scheme consists of restricting the superintegral (148) to the subset of supermatrices $\left(Z_{0}, \tilde{Z}_{0}\right)$ around which the first variations

$$
\lim _{\eta \rightarrow 0} \frac{S_{0}\left[Z_{0}+\eta W, \tilde{Z}_{0}\right]-S_{0}\left[Z_{0}, \tilde{Z}_{0}\right]}{\eta} \text { and } \lim _{\eta \rightarrow 0} \frac{S_{0}\left[Z_{0}, \tilde{Z}_{0}+\eta W\right]-S_{0}\left[Z_{0}, \tilde{Z}_{0}\right]}{\eta}
$$

of the exact source-free action $S_{0}[Z, \tilde{Z}]$ vanish as $\epsilon \rightarrow 0$ for all supermatrices $W$ in $L(T R \otimes \mathcal{A} \mid T R \otimes \mathcal{A})$. This subset of mean field configurations, called the zero mode, was identified in [22] and [23] and consists of the supermatrices satisfying

$$
\begin{gathered}
Z_{0}=\mathbb{1}_{\mathcal{A}} \otimes Y \quad \text { and } \quad \tilde{Z}_{0}=\mathbb{1}_{\mathcal{A}} \otimes \tilde{Y}, \\
\text { with } \quad Y, \tilde{Y} \in L(T R \mid T R) \quad \text { such that } \quad \tilde{Y}=Y^{\tau} .
\end{gathered}
$$

Moreover, $Y$ and $\tilde{Y}$ must be diagonal in $T R$ space if time-reversal symmetry is broken. Of course, the color-flavor relations (144) must still be satisfied, that is, the identities $\tilde{Y}_{\mathrm{BB}}=Y_{\mathrm{BB}}^{\dagger}$ and $\tilde{Y}_{\mathrm{FF}}=-Y_{\mathrm{FF}}^{\dagger}$ are fulfilled, and the eigenvalues of $Y_{\mathrm{BB}}^{\dagger} Y_{\mathrm{BB}}$ must have moduli smaller than one. The supermatrices $(Y, \tilde{Y})$ satisfying these relations parametrize a manifold, the so-called Efetov $\sigma$ model space. Efetov's $\sigma$ model space with unitary symmetry has 4 commuting and 4 anticommuting parameters, whereas 8 commuting and 8 anticommuting parameters are involved in the orthogonal symmetry class.

Let us introduce a 2 -dimensional $\mathbb{C}$-linear space $R A$, called retarded-advanced space, and let us consider the supermatrices in $L(R A \otimes T R \mid R A \otimes T R)$

$$
R \equiv\left(\begin{array}{cc}
1 & Y \\
\tilde{Y} & 1
\end{array}\right)_{R A} \quad \text { and } \quad R^{-1}=\left(\begin{array}{cc}
\frac{1}{1-Y \tilde{Y}} & -Y \frac{1}{1-\tilde{Y} Y} \\
-\tilde{Y} \frac{Y}{1-Y \tilde{Y}} & \frac{1}{1-\tilde{Y} Y}
\end{array}\right)_{R A}
$$


Then, one can set [51]

$$
Q \equiv R \sigma_{3}^{R A} R^{-1},
$$

where $\sigma_{3}^{R A}$ stands for the third Pauli matrix in retarded-advanced space. By construction, these matrices satisfy $Q^{2}=Q$. Moreover, if for a supermatrix $A$ having a retardedadvanced structure $\bar{A}$ denotes the supermatrix

$$
\bar{A} \equiv \mathcal{K} A^{\dagger} \mathcal{K}, \quad \text { where } \quad \mathcal{K} \equiv\left(\begin{array}{cc}
\sigma_{3}^{R A} & 0 \\
0 & 1
\end{array}\right)_{B F},
$$

the Efetov $\sigma$ model space is characterized by the constraints $\bar{Q}=Q, Q^{\tau}=\sigma_{3}^{R A} Q \sigma_{3}^{R A}$, and $Q$ diagonal in $T R$ space for the unitary symmetry class. Efetov's polar coordinates [19] then involve writing

$$
Q=U Q_{0} \bar{U}
$$

with

$$
Q_{0} \equiv\left(\begin{array}{cc}
\cos \hat{\theta} & i \sin \hat{\theta} \\
-i \sin \hat{\theta} & -\cos \hat{\theta}
\end{array}\right)_{R A}, \quad \hat{\theta} \equiv\left(\begin{array}{cc}
i \theta_{B} & 0 \\
0 & \theta_{F}
\end{array}\right)_{B F} .
$$

The equations $Q_{0}^{2}=Q_{0}$ and $\bar{Q}_{0}=Q_{0}$ are automatically fulfilled for any real symmetric matrices $\theta_{B}$ and $\theta_{F}$ acting on the $T R$ space if

$$
U \equiv U_{1} U_{2} \equiv\left(\begin{array}{cc}
u & 0 \\
0 & v
\end{array}\right)_{R A} \equiv\left(\begin{array}{cc}
u_{1} & 0 \\
0 & v_{1}
\end{array}\right)_{R A}\left(\begin{array}{cc}
u_{2} & 0 \\
0 & v_{2}
\end{array}\right)_{R A}
$$

are required to satisfy $\bar{U}_{1} U_{1}=1$ and $\bar{U}_{2} U_{2}=1$, that is $\bar{u}_{i} \equiv u_{i}^{\dagger}=u_{i}^{-1}$ and $\bar{v}_{i} \equiv$ $\sigma_{3}^{B F} v_{i}^{\dagger} \sigma_{3}^{B F}=v_{i}^{-1}$, for $i \in\{1,2\}$. The purpose of $U_{1}$ is to diagonalize $Q$ in Bose-Fermi space, and hence, this supermatrix contains all the anticommuting parameters. One can for example choose

$$
u_{1} \equiv\left(\begin{array}{cc}
1-2 \eta^{\dagger} \eta+6\left(\eta^{\dagger} \eta\right)^{2} & -2\left(1-2 \eta^{\dagger} \eta\right) \eta^{\dagger} \\
2 \eta\left(1-2 \eta^{\dagger} \eta\right) & 1-2 \eta \eta^{\dagger}+6\left(\eta \eta^{\dagger}\right)^{2}
\end{array}\right)_{B F}, \quad \eta \equiv\left(\begin{array}{cc}
\eta_{1}^{*} & \eta_{2} \\
\eta_{2}^{*} & \eta_{1}
\end{array}\right)_{T R},
$$

with $\eta_{i}, \eta_{i}^{*} \in \Lambda_{F}, i \in\{1,2\}$, and define $v_{1}$ by substituting $i \kappa_{1}$ for $\eta_{1}$ and $i \kappa_{2}$ for $\eta_{2}$. For the Efetov space with unitary symmetry, one sets $\eta_{2}, \kappa_{2} \rightarrow 0$, in which case $\eta \eta^{\dagger} \eta$ and $\eta^{\dagger} \eta \eta^{\dagger}$ vanish, and similarly for $\kappa$ and $\kappa^{\dagger}$. Requiring $Q_{0}$ to carry the additional symmetry $Q_{0}^{\tau}=\sigma_{3}^{R A} Q_{0} \sigma_{3}^{R A}$ amounts to writing the matrix angles $\theta_{B}$ and $\theta_{F}$ in (156) as

$$
\theta_{B}=\left(\begin{array}{cc}
\theta_{1} & \theta_{2} \\
\theta_{2} & \theta_{1}
\end{array}\right)_{T R}, \quad \theta_{F}=\left(\begin{array}{cc}
\theta & 0 \\
0 & \theta
\end{array}\right)_{T R},
$$

with $\theta_{1}, \theta_{2}>0$ and $\theta \in[0,2 \pi]$, and set $\theta_{2} \rightarrow 0$ in the unitary symmetry case. Together with the property $U_{1}^{\tau}=\bar{U}_{1}$ which follows from the definitions of $u_{1}$ and $v_{1}$ above, and from imposing $U_{2}^{\tau}=\bar{U}_{2}$ on $U_{2}$, this symmetry implies that the required equality $Q^{\tau}=\sigma_{3}^{R A} Q \sigma_{3}^{R A}$ indeed holds. There are still 2 and 5 remaining commuting parameters that have to be included in $U_{2}$ in order to span the full Efetov space for unitary and orthogonal symmetries respectively. It is not difficult to check that, for any matrix $V$ in $\mathrm{SU}(2)$, and for any $\xi, \chi \in[0,2 \pi]$,

$$
u_{2} \equiv\left(\begin{array}{cc}
e^{i \xi \sigma_{3}^{T R}} & 0 \\
0 & V
\end{array}\right)_{B F} \quad \text { and } \quad v_{2} \equiv\left(\begin{array}{cc}
e^{i \chi \sigma_{3}^{T R}} & 0 \\
0 & 1
\end{array}\right)_{B F}
$$


lead to a supermatrix $U_{2}$ in 157 with the required symmetries $\bar{U}_{2}=U_{2}^{\tau}=U_{2}^{-1}$. This finishes the description of the Efetov space with orthogonal symmetry in terms of polar coordinates. In the unitary symmetry case, one can take $\chi=0$ and $V=e^{i \phi \sigma_{3}^{T R}}$.

\subsection{The Mean Field Autocorrelation Functions}

The restriction of the superintegral (148) onto the zero mode defines mean field generating functions $\xi_{[\alpha]}^{\mathrm{MF}}(j)$ which, using (152) and (153), and after some algebra, can be put on the form

$$
\xi_{[\boldsymbol{\alpha}]}^{\mathrm{MF}}(\boldsymbol{j}) \equiv \int d Q e^{-S_{0}^{\mathrm{MF}}[Q]} P_{[\boldsymbol{\alpha}]}(\boldsymbol{j})
$$

where

$$
S_{0}^{\mathrm{MF}}[Q]=\frac{B \epsilon}{2} \operatorname{str} \hat{Q}+O(\epsilon)
$$

is the source-free action $S_{0}$ at the configuration $Q$ of the zero mode, $\hat{Q}$ denotes the supermatrix $\sigma_{3}^{R A} Q-\mathbb{1}, \hat{Q}^{B}$ stands for its Bose-Bose block, and $P_{[\alpha]}(j)$ is the supersymmetry breaking factor

$$
P_{[\alpha]}(\boldsymbol{j})=\operatorname{det}\left[\mathbb{1}-\frac{1}{2}\left(\begin{array}{cc}
\boldsymbol{j}_{\boldsymbol{r}} \cdot \mathcal{E}^{(\boldsymbol{r})} & 0 \\
0 & j_{a} \mathcal{E}^{(a)}
\end{array}\right)_{R A} \hat{Q}^{B}\right]^{-\frac{1}{2}}
$$

In this last expression $\mathcal{E}^{(a)}$ is the time-reversal double of $E^{(a)}$ in (105), and similarly $\mathcal{E}^{(r)}$ is the vector containing the time-reversal doubles of the $q-1$ matrices entering the vector $\boldsymbol{E}^{(r)}$, and in 161, $d Q$ is the measure $d(Z, \tilde{Z})$ in (148) induced on the zero mode manifold. Notice that the scattering matrix $S$ does not enter the mean field generating function. It can indeed be seen in (149) that, after being commuted with $Z_{0}$ and $\tilde{Z}_{0}, S$ meets its adjoint in the mean field action and thus disappears by unitarity.

The formula (110) applied to the mean field generating functions instead of the exact ones generates mean field autocorrelation functions $C_{[\alpha]}^{\mathrm{MF}}$, and commuting the derivatives with respect to the sources with the superintegral in 161) yields

$$
C_{[\alpha]}^{\mathrm{MF}}=\lim _{\epsilon \rightarrow 0} \frac{(2 \epsilon)^{q-1}}{2 B(q-1) !} \int d Q e^{-S_{0}^{\mathrm{MF}}} \delta P_{[\alpha]},
$$

where $\delta P_{[\alpha]}$ denotes the derivatives

$$
\delta P_{[\alpha]} \equiv\left(\prod_{s=0}^{q-1} \frac{\partial}{\partial j_{s}}\right) P_{[\alpha]}(\mathbf{0}) .
$$

These derivatives can easily be calculated by means of the general rule (109). For any integer $q \geq 2$, one gets

$$
\delta P_{[\alpha]}=\frac{1}{2^{q}} \sum_{\sigma \in S_{q}} \rho_{\frac{1}{2}}(\sigma) \sum_{t \in\{\uparrow, \downarrow\}^{q}} F_{[\alpha]}(\boldsymbol{t}, \sigma) \pi(\boldsymbol{t}, \sigma),
$$


where, for $\alpha \in \mathbb{R}$ and $\sigma \in S_{q}, \rho_{\alpha}(\sigma)$ denotes the $\rho$ factor defined in 108), and for any vector $\boldsymbol{t} \in\{\uparrow, \downarrow\}^{q}$,

$$
\pi(\boldsymbol{t}, \sigma) \equiv\left\{\begin{array}{cc}
\hat{Q}_{a a ; t_{0}, t_{0}}^{B} \prod_{j=1}^{q-1} \hat{Q}_{r r ; t_{j}, t_{\sigma(j)}}^{B} & \text { if } \sigma(0)=0 \\
\hat{Q}_{a r ; t_{0}, t_{\sigma(0)}}^{B} \hat{Q}_{r a ; t_{i}, t_{0}}^{B} \prod_{\substack{\text { s } \\
j \neq 1}}^{q-1} \hat{Q}_{r r ; t_{j}, t_{\sigma(j)}}^{B} & \text { if } \sigma(i)=0, i \in \mathbb{N}_{q-1}
\end{array}\right.
$$

involves a product of $q$ components of the $Q$ matrix and

$$
F_{[\alpha]}(\boldsymbol{t}, \sigma) \equiv \sum_{\gamma \in\left(\mathbb{N}_{2 B}\right)^{q}} \prod_{j=0}^{q-1}\left[\mathcal{E}^{\alpha_{j}, \alpha_{j}}\right]_{\substack{\gamma_{j}, \gamma_{\sigma(j)} \\ t_{j}, t_{j}}}=\prod_{j=0}^{q-1} \delta_{t_{j}\left[\alpha_{j}\right], t_{\sigma(j)}\left[\alpha_{\sigma(j)}\right]},
$$

is the $[\alpha]$-dependent factor. In (168), the new notations $\uparrow[\beta]=\beta$ and $\downarrow[\beta]=\hat{\beta}$ for a directed bond $\beta \in \mathbb{N}_{2 B}$ have been introduced. These results enable us to rewrite the mean field autocorrelation functions (164) as

$$
C_{[\alpha]}^{\mathrm{MF}}=\sum_{\sigma \in S_{q}} \rho_{\frac{1}{2}}(\sigma) \sum_{t \in\{\uparrow, \downarrow\} q} F_{[\alpha]}(\boldsymbol{t}, \sigma) I_{\pi}(\boldsymbol{t}, \sigma),
$$

where, for any $\boldsymbol{t}=\left(t_{0}, \ldots, t_{q-1}\right)$ in $\{\uparrow, \downarrow\}^{q}$, and for any $\sigma \in S_{q}$,

$$
I_{\pi}(\boldsymbol{t}, \sigma) \equiv \lim _{\epsilon \rightarrow 0} \frac{\epsilon^{q-1}}{4 B(q-1) !} \int d Q e^{-S_{0}^{\mathrm{MF}}} \pi(\boldsymbol{t}, \sigma) .
$$

The superintegrals $I_{\pi}(t, \sigma)$ are mean field superintegrals in the $\epsilon \rightarrow 0$ regime, and their values depend on the symmetry class. In the unitary symmetry class, the measure $d Q$ reads [19]

$$
d Q=\frac{1}{2^{6} \pi^{2}} \frac{d \lambda_{1} d \lambda}{\left(\lambda_{1}-\lambda\right)^{2}} d \eta_{1} d \eta_{1}^{*} d \kappa_{1}^{*} d \kappa_{1} d \phi d \xi
$$

in terms of Efetov's polar coordinates, where $\lambda_{1} \equiv \cosh \theta_{1}$ and $\lambda \equiv \cos \theta$, and the mean field source-free action $S_{0}^{\mathrm{MF}}$ is

$$
S_{0}^{\mathrm{MF}}=2 B \epsilon\left(\lambda_{1}-\lambda\right) .
$$

It can be checked, and it is stated in [36], that, in the unitary mean field superintegral (170), the lowest order term in $\epsilon$ is obtained by only retaining in $\pi(t, \sigma)$ its terms of highest order in $\lambda_{1}$ and by replacing the expression $\left(\lambda_{1}-\lambda\right)$ in (171) and (172) with $\lambda_{1}$. Therefore, in the expressions

$$
\begin{aligned}
\hat{Q}_{r r}^{B} & =u_{1 B B} u_{2 B} \cosh \theta_{B} \bar{u}_{2 B} \bar{u}_{1 B B}+u_{1 B F} u_{2 F} \cos \theta_{F} \bar{u}_{2 F} \bar{u}_{1 F B}-1 \\
\hat{Q}_{a a}^{B} & =v_{1 B B} v_{2 B} \cosh \theta_{B} \bar{v}_{2 B} \bar{v}_{1 B B}+v_{1 B F} v_{2 F} \cos \theta_{F} \bar{v}_{2 F} \bar{v}_{1 F B}-1 \\
\hat{Q}_{r a}^{B} & =-u_{1 B B} u_{2 B} \sinh \theta_{B} \bar{v}_{2 B} \bar{v}_{1 B B}+u_{1 B F} u_{2 F} i \sin \theta_{F} \bar{v}_{2 F} \bar{v}_{1 F B} \\
\hat{Q}_{a r}^{B} & =-v_{1 B B} v_{2 B} \sinh \theta_{B} \bar{u}_{2 B} \bar{u}_{1 B B}+v_{1 B F} v_{2 F} i \sin \theta_{F} \bar{u}_{2 F} \bar{u}_{1 F B}
\end{aligned}
$$

for the components of $\hat{Q}^{B}$, which follow from (155), (156) and (157), only the first terms of the right-hand sides contribute in the limit (170). Moreover, for the same 
reason one can replace $\sinh \theta_{1}$ with $\cosh \theta_{1}$. These remarks, together with the formulae (158), (159) and (160), lead to

$$
\hat{Q}^{B} \sim U_{2 B} \cdot x \cdot U_{2 B}^{\dagger}
$$

with

$$
x \equiv \lambda_{1} \cdot\left(\begin{array}{cc}
|\eta|^{2} & -|\eta||\kappa| \\
-|\eta||\kappa| & |\kappa|^{2}
\end{array}\right)_{R A}, \quad U_{2 B} \equiv\left(\begin{array}{cc}
e^{i \xi \sigma_{3}^{T R}} & 0 \\
0 & e^{i \chi \sigma_{3}^{T R}}
\end{array}\right)_{R A},
$$

and

$$
|\eta| \equiv 1-2 \eta_{1}^{*} \eta_{1}, \quad|\kappa| \equiv 1+2 \kappa_{1}^{*} \kappa_{1}
$$

In (174) and henceforth, for $a$ and $b$ two functions of the Efetov polar coordinates, the equivalence $a \sim b$ means that $b$ can be substituted for $a$ in the integrand of the mean field integral without modifying the result. The equivalence (174) implies that $\pi(t, \sigma)$ in (167) satisfies

$$
\begin{aligned}
\pi(\boldsymbol{t}, \sigma) & \sim \prod_{j=0}^{q-1} \delta_{\left.t_{j}, t_{\sigma(j)}\right)} \begin{cases}x_{a a} x_{r r}^{q-1} & \text { if } \sigma(0)=0 \\
x_{a r} x_{r a} x_{r r}^{q-2} & \text { if } \sigma(i)=0, i \in \mathbb{N}_{q-1}\end{cases} \\
& \sim|\eta|^{2(q-1)}|\kappa|^{2} \cdot \lambda_{1}^{q} \cdot \prod_{j=0}^{q-1} \delta_{t_{j}, t_{\sigma(j)}} \\
& \sim 2^{4}(q-1) \cdot \kappa_{1} \kappa_{1}^{*} \eta_{1}^{*} \eta_{1} \cdot \lambda_{1}^{q} \cdot \prod_{j=0}^{q-1} \delta_{t_{j}, t_{\sigma(j)}}
\end{aligned}
$$

The last equivalence expresses the fact that only the term containing all the anticommuting parameters can contribute to the superintegral (170). Combining (170), (171) and (172) with $\left(\lambda_{1}-\lambda\right) \rightarrow \lambda_{1}$, and (177) together, one arrives at

$$
I_{\pi}(\boldsymbol{t}, \sigma)=\lim _{\epsilon \rightarrow 0} \frac{\epsilon^{q-1}}{2 B(q-2) !} \int_{0}^{\infty} e^{-2 B \epsilon \lambda_{1}} \lambda_{1}^{q-2} d \lambda_{1} \cdot \prod_{j=0}^{q-1} \delta_{t_{j}, t_{\sigma(j)}}=\frac{1}{(2 B)^{q}} \cdot \prod_{j=0}^{q-1} \delta_{t_{j}, t_{\sigma(j)}}
$$

In the orthogonal symmetry class, a similar calculation leads to $I_{\pi}(t, \sigma)=(2 B)^{-q}$. This result can also be inferred from (169), (178), and from the expectation that the mean field intensity correlation matrix should satisfy $C_{\alpha \alpha^{\prime}}^{\mathrm{MF}}=C_{\hat{\alpha} \alpha^{\prime}}^{\mathrm{MF}}$ and should not depend on the symmetry class if $\alpha$ and $\alpha^{\prime}$ are supported on two different bonds.

By 169 and by the results found above for $I_{\pi}(t, \sigma)$, the mean field autocorrelation functions become

$$
C_{[\alpha]}^{\mathrm{MF}}=\frac{1}{(2 B)^{q}} \sum_{\substack{\sigma \in S_{q} \\ t \in\left\{\uparrow, \downarrow^{q}\right.}} \rho_{\frac{1}{2}}(\sigma) \prod_{j=0}^{q-1} \begin{cases}\delta_{t_{j}, t_{\sigma(j)}} \delta_{\alpha_{j}, \alpha_{\sigma(j)}} & (U) \\ \delta_{t_{j}\left[\alpha_{j}\right], t_{\sigma(j)}\left[\alpha_{\sigma(j)}\right]} & (O)\end{cases}
$$

In order to get some explicit formulae out of [179], one can for example apply the rule (109) once more, and notice that

$$
C_{[\alpha]}^{\mathrm{MF}}=\left.\frac{1}{(2 B)^{q}}\left(\prod_{k=0}^{q-1} \frac{\partial}{\partial j_{k}}\right) \operatorname{det}\left[1-\sum_{k=0}^{q-1} j_{k} N\left(\alpha_{k}\right)\right]^{-\frac{1}{2}}\right|_{j=\mathbf{0}},
$$


where, for $\beta \in \mathbb{N}_{2 B}$ a directed bond, $N(\beta)$ is the matrix acting on $\mathcal{A} \otimes T R$ defined by

$$
N(\beta) \equiv\left\{\begin{array}{cc}
E^{\beta, \beta} \otimes \mathbb{1}_{T R} & (U) \\
E^{\beta, \beta} & E^{\beta, \beta} \\
E^{\hat{\beta}, \hat{\beta}} & E^{\hat{\beta}, \hat{\beta}}
\end{array}\right)_{T R} \quad(O)
$$

Let us first consider the unitary symmetry class $(U)$, and let us characterize the list $[\alpha]$ of directed bonds by another list $\beta=\left(\beta_{1}, \ldots, \beta_{n}\right)$ of distinct directed bonds and a vector of integers $\boldsymbol{q}=\left(q_{1}, \ldots, q_{n}\right)$ such that $\beta_{j}$ occurs exactly $q_{j}$ times in $[\alpha]$. With the notation $C_{\beta}^{\mathrm{MF}}(\boldsymbol{q})=C_{[\alpha]}^{\mathrm{MF}}$, an explicit calculation of the determinant in (180) shows that

$$
C_{\boldsymbol{\beta}}^{\mathrm{MF}}(\boldsymbol{q})=\left.\frac{1}{(2 B)^{q}} \prod_{k=1}^{n} \frac{\partial^{q_{k}}}{\partial j_{k}^{q_{k}}} \frac{1}{1-j_{k}}\right|_{j_{k}=0}=\prod_{j=1}^{n} \frac{q_{j} !}{(2 B)^{q_{j}}} .
$$

Let us now consider the orthogonal symmetry class $(O)$, and let us characterize the list $[\alpha]$ by the list $\boldsymbol{\beta}$ of distinct directed bonds and the vector $\boldsymbol{q}$ of integers

$$
\left\{\begin{array}{l}
\boldsymbol{\beta}=\left(\boldsymbol{\beta}_{1}, \ldots, \boldsymbol{\beta}_{m}, \beta_{m+1}, \ldots, \beta_{n}\right) \\
\boldsymbol{q}=\left(\boldsymbol{q}_{1}, \ldots, \boldsymbol{q}_{m}, q_{m+1}, \ldots, q_{n}\right)
\end{array}, \quad\left\{\begin{array}{l}
\boldsymbol{\beta}_{j} \equiv\left(\beta_{j}, \hat{\beta}_{j}\right) \\
\boldsymbol{q}_{j} \equiv\left(q_{j}, \hat{q}_{j}\right)
\end{array}, j \in \mathbb{N}_{m}\right.\right.
$$

such that the components of $\boldsymbol{q}$ indicate the number of occurrences of the corresponding elements in $\beta$. A first inspection of the formula $\left(180\right.$ ) shows that $C_{\beta}^{\mathrm{MF}}(\boldsymbol{q})$ factorizes as

$$
C_{\beta}^{\mathrm{MF}}(\boldsymbol{q})=\prod_{k=1}^{m} C_{\beta_{k}, \hat{\beta}_{k}}^{\mathrm{MF}}\left(q_{k}, \hat{q}_{k}\right) \prod_{k=m+1}^{n} C_{\beta_{k}}^{\mathrm{MF}}\left(q_{k}\right)
$$

Then, a calculation of the determinant shows that the correlation functions $C_{\beta_{k}}^{\mathrm{MF}}\left(q_{k}\right)$ are given by the unitary formula (182), and

$$
C_{\beta, \hat{\beta}}^{\mathrm{MF}}(q, \hat{q})=\left.\frac{1}{(2 B)^{q+\hat{q}}} \frac{\partial^{q}}{\partial j^{q}} \frac{\partial^{\hat{q}}}{\partial \hat{j}^{\hat{q}}} \frac{1}{1-j-\hat{j}}\right|_{j=\hat{j}=0}=\frac{(q+\hat{q}) !}{(2 B)^{q+\hat{q}}}
$$

It can be checked that these formulae coincide precisely with the predictions of the Gaussian Random Waves Models (48) and (53).

Moreover, it can be checked in (179) that the autocorrelation functions $C_{[\alpha]}^{\sigma}$ defined at the end of Subsection 4.1 give rise to mean field autocorrelation functions $C_{[\alpha]}^{\sigma, \mathrm{MF}}$ that do not depend on the particular convention $\sigma \in S_{q}$ chosen.

\section{The Gaussian Correction}

\subsection{Beyond Mean Field Theory}

It is known [11] that not all increasing sequences of quantum graphs are quantum ergodic, and hence, the mean field theory does not always yield the main contributions to the autocorrelation functions. Therefore, it is necessary to estimate the importance 
of the supermatrices $Z$ and $\tilde{Z}$ lying off the zero mode manifold. For this purpose, let us write

$$
Z \equiv Z_{0}+\delta Z, \quad \tilde{Z} \equiv \tilde{Z}_{0}+\delta \tilde{Z},
$$

with $\left(Z_{0}, \tilde{Z}_{0}\right)$ on the zero mode manifold and with $(\delta Z, \delta \tilde{Z})$ an orthogonal deviation, and let us expand the exact action $S[Z, \tilde{Z}]$ in $(149)$ up to second order in $\delta Z$ and $\delta \tilde{Z}$ around $\left(Z_{0}, \tilde{Z}_{0}\right)$. The truncated action $\tilde{S}\left[\delta Z, \delta \tilde{Z}, Z_{0}, \tilde{Z}_{0}\right]$ obtained in this way leads to a generating function

$$
\int d\left(Z_{0}, \tilde{Z}_{0}\right) d(\delta Z, \delta \tilde{Z}) e^{-\tilde{S}\left[\delta Z, \delta \tilde{Z} ; Z_{0}, \tilde{Z}_{0}\right]} .
$$

Suppose for the moment that $Z$ and $\tilde{Z}$ in (187) are also required to satisfy $\tilde{Z}=Z^{\tau}$. It follows that the partial traces over $\mathcal{A}$ of the supermatrices $\delta Z$ and $\delta \tilde{Z}$ in (186) must vanish. This property implies that the truncated action $\tilde{S}$ has no linear terms in $\delta Z$ and $\delta \tilde{Z}$. A direct but tedious calculation shows that, if the sources in the truncated action are set to zero, one gets

$$
\tilde{S}_{0}\left[\delta Z, \delta \tilde{Z} ; Z_{0}, \tilde{Z}_{0}\right]=S_{0}^{\mathrm{MF}}\left[Z_{0}, \tilde{Z}_{0}\right]+S_{0}^{(2)}[W ; \tilde{W}],
$$

where $S_{0}^{\mathrm{MF}}\left[Z_{0}, \tilde{Z}_{0}\right]$ is the mean field source-free action,

$$
W \equiv\left(1-Z_{0} \tilde{Z}_{0}\right)^{-\frac{1}{2}} \delta Z\left(1-\tilde{Z}_{0} Z_{0}\right)^{-\frac{1}{2}}, \quad \tilde{W} \equiv\left(1-\tilde{Z}_{0} Z_{0}\right)^{-\frac{1}{2}} \delta \tilde{Z}\left(1-Z_{0} \tilde{Z}_{0}\right)^{-\frac{1}{2}},
$$

and $S_{0}^{(2)}[W, \tilde{W}]$ is the term of the exact source-free action $S_{0}[W, \tilde{W}]$ of second order in $W$ and $\tilde{W}$ around the origin. If $e^{-\tilde{S}_{0}}$ was to be integrated as in (187), the changes of variables (189), which both have unit Jacobian, would factorize the superintegrals over the zero mode $\left(Z_{0}, \tilde{Z}_{0}\right)$ and over the orthogonal deviation $(W, \tilde{W})$. This factorization occurs because the domain of the superintegral over $(W, \tilde{W})$ is independent of $\left(Z_{0}, \tilde{Z}_{0}\right)$. Indeed, it can be readily seen that the equality $\tilde{Z}=Z^{\tau}$ merely becomes $\tilde{W}=W^{\tau}$. Moreover, if all the Grassmann generators of $\Lambda$ are sent to zero, which is what really matters for the domain of a superintegral, the color-flavor requirements (144) on $(\delta Z, \delta \tilde{Z})$ become $\tilde{W}_{B B}=W_{B B}^{\dagger}$ and $\tilde{W}_{F F}=-W_{F F}^{\dagger}$, and there is no further condition concerning the eigenvalues of the positive Hermitian matrix $W_{B B}^{\dagger} W_{B B}$. Finally, the condition ensuring that $(\delta Z, \delta \tilde{Z})$ is orthogonal to the zero mode manifold forces $W$ and $\tilde{W}$ to have vanishing partial traces over $\mathcal{A}$.

By analogy with the situation described above, where the sources are set to zero and where the supermatrix variables are constrained to satisfy $\tilde{Z}=Z^{\tau}$, one defines the truncated generating functions

$$
\tilde{\xi}_{[\alpha]}(j) \equiv \xi_{[\alpha]}^{\mathrm{MF}}(j) \cdot \xi_{[\alpha]}^{G}(j),
$$

where the Gaussian generating function is defined by

$$
\xi_{[\alpha]}^{G}(j) \equiv \int d^{G}(W, \tilde{W}) e^{-S^{(2)}[W, \tilde{W}]}
$$

and $S^{(2)}[W, \tilde{W}]$ is the term of the exact action $S[W, \tilde{W}]$ in 149 ) of second order in $W$ and $\tilde{W}$ around the origin, namely

$$
S^{(2)}[W, \tilde{W}]=\operatorname{str}\left(W \tilde{W}-\frac{1}{2} \mathcal{J}_{r} \tilde{W}^{\tau} \mathcal{J}_{a} \tilde{W}-\frac{1}{2} W \mathcal{S}_{\epsilon}^{\dagger} W^{\tau} \mathcal{S}_{\epsilon}\right)
$$


The integration in (191) is over all supermatrices $W$ and $\tilde{W}$ in $L(T R \otimes \mathcal{A l} T R \otimes \mathcal{A})$ that are diagonal in $\mathcal{A}_{b}$, define a configuration $(W, \tilde{W})$ orthogonal to the zero mode manifold, and satisfy the color-flavor conditions $\tilde{W}_{B B}=W_{B B}^{\dagger}$ and $\tilde{W}_{F F}=-W_{F F}^{\dagger}$. The measure $d^{G}(W, \tilde{W})$ is then the product of the flat Berezin measures over the independent components of $W$ and $\tilde{W}$.

In (112), it is claimed that if either the advanced or the retarded sources are sent to zero, the exact generating function becomes identically equal to one in a neighborhood of the origin. It can be checked that the same property holds separately for the mean field and the Gaussian generating functions. Therefore, if the formula (110) is used to define truncated autocorrelation functions $\tilde{C}_{[\alpha]}$ from $\tilde{\xi}_{[\alpha]}$, one gets

$$
\tilde{C}_{[\alpha]}=C_{[\alpha]}^{\mathrm{MF}}+C_{[\alpha]}^{G},
$$

where $C_{[\alpha]}^{\mathrm{MF}}$ are the mean field autocorrelation functions found in the previous section, and

$$
C_{[\alpha]}^{G}=\lim _{\epsilon \rightarrow 0} \frac{(2 \epsilon)^{q-1}}{2 B(q-1) !} \delta \xi_{[\alpha]}^{G}, \quad \delta \xi_{[\alpha]}^{G} \equiv\left(\prod_{s=0}^{q-1} \frac{\partial}{\partial j_{s}}\right) \xi_{[\alpha]}^{G}(\mathbf{0}),
$$

are the Gaussian autocorrelation functions.

In fact, in order to calculate the Gaussian generating functions [191, one can first calculate the second order generating function

$$
\xi_{[\alpha]}^{(2)}(j) \equiv \int d^{(2)}(Z, \tilde{Z}) e^{-S^{(2)}[Z, \tilde{Z}]},
$$

defined from (191) by relaxing the constraint that $(Z, \tilde{Z})$ must be orthogonal to the zero mode, and then divide by the second order mean field generating function

$$
\xi_{[\boldsymbol{\alpha}]}^{\mathrm{MF}(2)}(\boldsymbol{j}) \equiv \int d^{\mathrm{MF}(2)}(Y, \tilde{Y}) e^{-S^{\mathrm{MF}(2)[Y, \tilde{Y}]},}
$$

which contains the zero mode contribution to 195. With the notations $Z_{0}=\mathbb{1}_{\mathcal{A}} \otimes Y$ and $\tilde{Z}_{0}=\mathbb{1}_{\mathcal{A}} \otimes \tilde{Y}$ for the supermatrix variables in the zero mode, $d^{\mathrm{MF}(2)}(Y, \tilde{Y})$ is the measure induced by $d^{(2)}(Z, \tilde{Z})$ on the zero mode manifold. Similarly, the mean field second order action $S^{\mathrm{MF}(2)}$ is obtained by restricting $S^{(2)}$, that is

$$
S^{\mathrm{MF}(2)}[Y, \tilde{Y}]=\frac{1}{2} \operatorname{str}\left(\left(2-e^{-2 \epsilon}\right) \mathbb{1}_{\mathcal{A}} \otimes Y \tilde{Y}-\mathcal{J}_{r} Y \mathcal{J}_{a} \tilde{Y}\right) .
$$

The generating functions (195) and (196) are identically equal to one in a neighborhood of the origin if either the advanced or the retarded sources are set to zero. Therefore, the Gaussian autocorrelation functions can be written

$$
C_{[\alpha]}^{G}=\lim _{\epsilon \rightarrow 0} \frac{(2 \epsilon)^{q-1}}{2 B(q-1) !}\left(\delta \xi_{[\alpha]}^{(2)}-\delta \xi_{[\alpha]}^{\mathrm{MF}(2)}\right)
$$

with the obvious definitions for $\delta \xi_{[\alpha]}^{(2)}$ and $\delta \xi_{[\alpha]}^{\mathrm{MF}(2)}$. 


\subsection{Diagonal Modes in Direction Space}

Let us first only consider the subset of supermatrix variables $(Z, \tilde{Z})$ that are diagonal in the whole amplitude space $\mathcal{A}=\mathcal{A}_{b} \otimes \mathcal{A}_{d}$. If $d_{(d d)}^{(2)}(Z, \tilde{Z})$ denotes the measure induced from $d^{(2)}(Z, \tilde{Z})$ on this subset, the goal is to calculate the $(d d)$ second order generating functions

$$
\xi_{[\boldsymbol{\alpha}],(d d)}^{(2)}(\boldsymbol{j}) \equiv \int d_{(d d)}^{(2)}(Z, \tilde{Z}) e^{-S^{(2)}[Z, \tilde{Z}]}
$$

The diagonal modes $Z$ and $\tilde{Z}$ are parametrized in time-reversal space as follows,

$$
Z=\left(\begin{array}{cc}
Z_{1} & Z_{2}^{\dagger} \\
Z_{3}^{\mathcal{T}} \sigma_{3}^{\mathrm{BF}} & Z_{4}^{\dagger \mathcal{T}}
\end{array}\right) \quad \text { and } \quad \tilde{Z}=\left(\begin{array}{cc}
\tilde{Z}_{1} & \sigma_{3}^{\mathrm{BF}} \tilde{Z}_{3}^{\mathcal{T}} \\
\tilde{Z}_{2}^{\dagger} & \tilde{Z}_{4}^{\dagger \mathcal{T}^{\top}}
\end{array}\right)
$$

and their generalized transposes read

$$
Z^{\tau}=\left(\begin{array}{cc}
Z_{4}^{\dagger} & \sigma_{3}^{\mathrm{BF}} Z_{2}^{\dagger \mathcal{T}} \\
Z_{3} & Z_{1}^{\mathcal{T}^{2}}
\end{array}\right) \quad \text { and } \quad \tilde{Z}^{\tau}=\left(\begin{array}{cc}
\tilde{Z}_{4}^{\dagger} & \tilde{Z}_{3} \\
\tilde{Z}_{2}^{\dagger \mathcal{T}^{\mathcal{T}}} \sigma_{3}^{\mathrm{BF}} & \tilde{Z}_{1}^{\mathcal{T}}
\end{array}\right)
$$

The modes $Z_{2}^{\dagger}, \tilde{Z}_{2}^{\dagger}, Z_{3}$ and $\tilde{Z}_{3}$ are only considered if time-reversal invariance is conserved, so that $Z, \tilde{Z}$ and their generalized transposes all become diagonal in timereversal space. When these formulae are substituted into $S^{(2)}[Z, \tilde{Z}]$ given by (192), the diagonal modes in time-reversal space, which are indexed by 1 and 4, are coupled together, and do not mix with the off-diagonal ones indexed by 2 and 3 . After some algebra, one finds

$$
S_{(d d)}^{(2)}=S_{(d d)}^{(2) D}+(\kappa-1) S_{(d d)}^{(2) C}
$$

with the diffusion action $S_{(d d)}^{(2) D}$ and the cooperon action $S_{(d d)}^{(2) C}$ defined by

$$
\begin{aligned}
& S_{(d d)}^{(2) D}=\operatorname{str}\left(Z_{1} \tilde{Z}_{1}+Z_{4}^{\dagger} \tilde{Z}_{4}^{\dagger}-J_{r} \tilde{Z}_{4}^{\dagger} J_{a} \tilde{Z}_{1}-Z_{1} S_{\epsilon}^{\dagger} Z_{4}^{\dagger} S_{\epsilon}\right) \\
& S_{(d d)}^{(2) C}=\operatorname{str}\left(Z_{2}^{\dagger} \tilde{Z}_{2}^{\dagger}+Z_{3} \tilde{Z}_{3}-J_{r} \tilde{Z}_{3} J_{a}^{\mathcal{T}} \tilde{Z}_{2}^{\dagger}-Z_{2}^{\dagger} S_{\epsilon}^{\mathcal{T} \dagger} Z_{3} S_{\epsilon}\right)
\end{aligned}
$$

Hence, the generating functions $\xi_{[\alpha],(d d)}^{(2)}$ factorize as

$$
\xi_{[\alpha],(d d)}^{(2)}=\xi_{[\alpha],(d d)}^{(2) D} \cdot\left(\xi_{[\alpha],(d d)}^{(2) C}\right)^{K-1}
$$

where, for $\circ \in\{D, C\}$,

$$
\xi_{[\alpha],(d d)}^{(2) \circ}(\boldsymbol{j})=\int d_{(d d)}^{(2) \circ}(Z, \tilde{Z}) e^{-S_{(d d)}^{(2) \circ}[Z, \tilde{Z}]} .
$$

In 206, the diffusion measure is the product of the Berezin measures of the independent components in the supermatrix variables $Z_{1}, Z_{4}^{\dagger}, \tilde{Z}_{1}$ and $\tilde{Z}_{4}^{\dagger}$, and the cooperon measure is similarly formed with the independent components in $Z_{2}^{\dagger}, Z_{3}, \tilde{Z}_{2}^{\dagger}$ and $\tilde{Z}_{3}$.

Notice that the cooperon generating functions (206), which only exist if $S^{\mathcal{T}}=S$, can be obtained from the corresponding diffusion generating functions by replacing $J_{a}$ with $J_{a}^{\mathcal{T}}$. One can thus focus on the diffusion generating functions and infer the cooperon result from this remark. 
In order to perform the diffusion and cooperon integrals in 206, the supertraces in 203 and 204 must be explicitly expanded in Bose-Fermi space. Notice that the color-flavor conditions on the Bose-Bose and Fermi-Fermi components of $Z$ and $\tilde{Z}$ in (200) become $\tilde{Z}_{j B B}=Z_{j B B}^{\dagger}=Z_{j B B}^{*}$ and $\tilde{Z}_{j F F}=-Z_{j F F}^{\dagger}=Z_{j F F}^{*}$ for all $j \in \mathbb{N}_{4}$. Let us define for each directed bond $\beta \in \mathbb{N}_{2 B}$

$$
\begin{array}{ll}
\tilde{z}_{1 \overline{0} \beta}=\left(Z_{1 B B}^{*}, Z_{1 F F}^{*}\right)_{\beta} \quad, & z_{1 \overline{0} \beta}=\left(\begin{array}{c}
Z_{1 B B} \\
Z_{1 F F}
\end{array}\right)_{\beta}, \\
z_{4 \overline{0} \beta}^{\dagger}=\left(Z_{4 B B}^{*},-Z_{4 F F}^{*}\right)_{\beta} \quad, \quad \tilde{z}_{4 \overline{0} \beta}^{\dagger}=\left(\begin{array}{c}
Z_{4 B B} \\
-Z_{4 F F}
\end{array}\right)_{\beta} .
\end{array}
$$

The vectors $\tilde{z}_{1 \overline{0} \beta}$ and $z_{1 \overline{0} \beta}$ contain the commuting parameters of $\tilde{Z}_{1 \beta}$ and $Z_{1 \beta}$ respectively, and the vectors $z_{4 \overline{0} \beta}^{\dagger}$ and $\tilde{z}_{4 \overline{0} \beta}^{\dagger}$ contain those of $Z_{4 \beta}^{\dagger}$ and $\tilde{Z}_{4 \beta}^{\dagger}$. Similarly, the anticommuting variables of the diffusion action are arranged in the vectors

$$
\begin{array}{ll}
\tilde{z}_{1 \overline{1} \beta}=\left(\tilde{Z}_{1 B F}, \tilde{Z}_{1 F B}\right)_{\beta} \quad, & z_{1 \overline{1} \beta}=\left(\begin{array}{c}
Z_{1 B F} \\
Z_{1 F B}
\end{array}\right)_{\beta}, \\
z_{4 \overline{1} \beta}^{\dagger}=\left(Z_{4 B F}^{*}, Z_{4 F B}^{*}\right)_{\beta} \quad, \quad \tilde{z}_{4 \overline{1} \beta}^{\dagger}=\left(\begin{array}{c}
\tilde{Z}_{4 B F}^{*} \\
\tilde{Z}_{4 F B}^{*}
\end{array}\right)_{\beta} .
\end{array}
$$

Collecting the $2 B$ row-vectors $\tilde{z}_{1 \overline{0} \beta}$ (resp. $z_{4 \overline{0} \beta}^{\dagger}$ ) together, one can write a larger row vector $\tilde{z}_{1 \overline{0}}$ (resp. $z_{4 \overline{0}}^{\dagger}$ ). The column-vectors $z_{1 \overline{0}}$ and $\tilde{z}_{4 \overline{0}}^{\dagger}$ are formed similarly from $z_{1 \overline{0} \beta}$ and $\tilde{z}_{4 \overline{0} \beta}^{\dagger}$, and one proceeds in the same way with the anticommuting variables in (208). Let us also introduce a $2 B \times 2 B$ matrix $s$ defined from the Bose-Bose blocks of the source supermatrices $J_{a}$ and $J_{r}$ by

$$
s(\boldsymbol{j})_{\beta \beta^{\prime}} \equiv J_{a}\left(j_{a}\right)_{B B, \beta^{\prime} \beta} J_{r}\left(\boldsymbol{j}_{\boldsymbol{r}}\right)_{B B, \beta \beta^{\prime}} .
$$

A direct expansion of 203 in Bose-Fermi space then leads to

$$
S_{(d d) \overline{0}}^{(2) D}=\left(\tilde{z}_{1 \overline{0}}, z_{4 \overline{0}}^{\dagger}\right)\left(\begin{array}{cc}
\mathbb{1}_{\mathcal{A}} \otimes \mathbb{1}_{2 \times 2} & -\left(\begin{array}{c}
s\left(j_{a}, j_{r}\right) \\
s(0, \mathbf{0})
\end{array}\right) \\
-M_{\epsilon} \otimes \mathbb{1}_{2 \times 2} & \mathbb{1}_{\mathcal{A}} \otimes \mathbb{1}_{2 \times 2}
\end{array}\right)\left(\begin{array}{c}
z_{1 \overline{0}} \\
\tilde{z}_{4 \overline{0}}^{\dagger}
\end{array}\right)
$$

for the part of $S_{(d d)}^{(2) D}$ involving the commuting variables, and

$$
S_{(d d) \overline{1}}^{(2) D}=\left(\tilde{z}_{1 \overline{1}}, z_{4 \overline{1}}^{\dagger}\right)\left(\begin{array}{cc}
\mathbb{1}_{\mathcal{A}} \otimes\left(\begin{array}{cc}
0 & 1 \\
-1 & 0
\end{array}\right) & \left(\begin{array}{ll}
s\left(j_{a}, \mathbf{0}\right) & \\
& s\left(0, j_{r}\right)
\end{array}\right) \\
M_{\epsilon} \otimes \mathbb{1}_{2 \times 2} & \mathbb{1}_{\mathcal{A}} \otimes\left(\begin{array}{cc}
0 & 1 \\
-1 & 0
\end{array}\right)
\end{array}\right)\left(\begin{array}{c}
z_{1 \overline{1}} \\
\tilde{z}_{4 \overline{1}}^{\dagger}
\end{array}\right)
$$

for the part involving the anticommuting variables. Notice that these formulae depend on the scattering matrix $S_{\epsilon}=e^{-\epsilon} S$ only through the classical map $M_{\epsilon}=e^{-2 \epsilon} M$ it generates. It is straightforward to calculate the diffusion superintegral 206 from these quadratic forms, and the cooperon generating functions are found by substituting the matrix $J_{a}^{\mathcal{T}}$ for the matrix $J_{a}$ in the diffusion results. If $\circ \in\{D, C\}$, one gets

$$
\xi_{[\alpha],(d d)}^{(2) \circ}\left(j_{a}, j_{r}\right)=\frac{\operatorname{det}\left(\mathbb{1}_{\mathcal{A}}-s^{\circ}\left(j_{a}, \mathbf{0}\right) M_{\epsilon}\right) \operatorname{det}\left(\mathbb{1}_{\mathcal{A}}-s^{\circ}\left(0, \boldsymbol{j}_{\boldsymbol{r}}\right) M_{\epsilon}\right)}{\operatorname{det}\left(\mathbb{1}_{\mathcal{A}}-M_{\epsilon}\right) \operatorname{det}\left(\mathbb{1}_{\mathcal{A}}-s^{\circ}\left(j_{a}, \boldsymbol{j}_{\boldsymbol{r}}\right) M_{\epsilon}\right)},
$$


where $s^{D} \equiv s$ in 209], and $s^{C}$ is obtained from $s$ by replacing $J_{a}$ with $J_{a}^{\mathcal{T}}$.

In order to unveil the mean field contribution to [212], one restricts the superintegral in (199) to the zero mode $Z_{0}=\mathbb{1}_{\mathcal{A}} \otimes Y, \tilde{Z}_{0}=\mathbb{1}_{\mathcal{A}} \otimes \tilde{Y}$. The supermatrices $Y$ and $\tilde{Y}$ are required to satisfy $\tilde{Y}=Y^{\tau}$, and can thus be parametrized by

$$
Y=\left(\begin{array}{cc}
Y_{\mathrm{D}} & Y_{\mathrm{C}} \\
\tilde{Y}_{\mathrm{C}}^{\mathcal{T}} \sigma_{3}^{B F} & \tilde{Y}_{\mathrm{D}}^{\mathcal{T}}
\end{array}\right) \quad \text { and } \quad \tilde{Y}=\left(\begin{array}{cc}
\tilde{Y}_{\mathrm{D}} & \sigma_{3}^{B F} Y_{\mathrm{C}}^{\mathcal{T}} \\
\tilde{Y}_{\mathrm{C}} & Y_{\mathrm{D}}^{\mathcal{T}}
\end{array}\right)
$$

in time-reversal space. Then, the second order mean field action (197) splits into a diffusion part, containing the supermatrices $Y_{\mathrm{D}}$ and $\tilde{Y}_{\mathrm{D}}$, and a cooperon part, involving $Y_{\mathrm{C}}$ and $\tilde{Y}_{C}$. One gets

$$
\begin{aligned}
& S^{\mathrm{MF}(2) D}=\operatorname{str}\left(\left(2-e^{-2 \epsilon}\right) Y_{\mathrm{D}} \tilde{Y}_{\mathrm{D}} \otimes \mathbb{1}_{\mathcal{A}}-J_{r} Y_{\mathrm{D}} J_{a} \tilde{Y}_{\mathrm{D}}\right) \\
& S^{\mathrm{MF}(2) C}=\operatorname{str}\left(\left(2-e^{-2 \epsilon}\right) Y_{\mathrm{C}} \tilde{Y}_{\mathrm{C}} \otimes \mathbb{1}_{\mathcal{A}}-J_{r} Y_{\mathrm{C}} J_{a}^{\mathcal{T}} \tilde{Y}_{\mathrm{C}}\right) .
\end{aligned}
$$

It follows that the mean field contribution to (212) factorizes into a diffusion and a cooperon factor, as in 205. The expressions (214) can be developed in Bose-Fermi space, and the resulting quadratic forms have inverse superdeterminant

$$
\xi_{[\alpha]}^{\mathrm{MF}(2) \circ}\left(j_{a}, \boldsymbol{j}_{\boldsymbol{r}}\right)=\frac{\left(1-e^{-2 \epsilon}-\sigma^{\circ}\left(j_{a}, \mathbf{0}\right)\right)\left(1-e^{-2 \epsilon}-\sigma^{\circ}\left(0, \boldsymbol{j}_{r}\right)\right)}{\left(1-e^{-2 \epsilon}\right)\left(1-e^{-2 \epsilon}-\sigma^{\circ}\left(j_{a}, \boldsymbol{j}_{r}\right)\right)}, \quad \circ \in\{D, C\},
$$

where

$$
\sigma^{\circ}\left(j_{a}, \boldsymbol{j}_{r}\right) \equiv \frac{1}{2 B} \sum_{\beta, \beta^{\prime}=1}^{2 B} s^{\circ}\left(j_{a}, \boldsymbol{j}_{r}\right)_{\beta \beta^{\prime}}-1 .
$$

Notice that no index $(d d)$ has been added in the left-hand side of (216). The reason is that the zero mode supermatrices $Z_{0}$ and $\tilde{Z}_{0}$ are always diagonal in the direction space $\mathcal{A}_{d}$, and hence (216) is also the mean field contribution to the full second order generating functions 195.

The diffusion and cooperon generating functions in (212) and (216) become identically one in a neighborhood of the origin if either the advanced or the retarded sources are set to zero. Hence, the ( $d d)$ Gaussian autocorrelation functions defined as in (198) from the $(d d)$ second order generating functions (205) split

$$
C_{[\alpha],(d d)}^{G}=C_{[\alpha],(d d)}^{G, D}+(\kappa-1) C_{[\alpha],(d d)}^{G, C},
$$

where the diffusion and cooperon autocorrelation functions in the right-hand side are defined by the formula (198) applied to the diffusion and cooperon versions of (212), that is

$$
C_{[\alpha],(d d)}^{G \circ} \equiv \lim _{\epsilon \rightarrow 0} \frac{(2 \epsilon)^{q-1}}{2 B(q-1) !}\left(\delta \xi_{[\alpha],(d d)}^{(2) \circ}-\delta \xi_{[\alpha]}^{\mathrm{MF}(2) \circ}\right), \quad \circ \in\{D, C\} .
$$

The next step towards the calculation of $(219)$ is to calculate the derivatives of the diffusion and cooperon $(d d)$ second order generating functions $(212)$. Performing 
explicitly the unique advanced derivative, one easily finds that for all integers $q \geq 2$, and for all sets of $q$ directed bonds $[\alpha]$,

$$
\delta \xi_{[\alpha],(d d)}^{(2) \circ}=\left.\prod_{s=1}^{q-1} \frac{\partial}{\partial j_{s}} \operatorname{tr}\left[\frac{\partial s^{\circ}}{\partial j_{a}}\left(0, j_{r}\right) M_{\epsilon} \frac{1}{1-s^{\circ}\left(0, j_{r}\right) M_{\epsilon}}\right]\right|_{j_{r}=\mathbf{0}},
$$

where $\boldsymbol{j}_{\boldsymbol{r}} \equiv\left(j_{1}, \ldots, j_{q-1}\right)^{T}$ are the $q-1$ retarded sources.

Let us first consider the situation $q=2$ and $[\alpha]=\left[\alpha, \alpha^{\prime}\right]$. Then, the unique retarded derivative in 220 can be performed, and one gets

$$
\delta \xi_{\left[\alpha, \alpha^{\prime}\right],(d d)}^{(2) \circ}=\operatorname{tr}\left[\frac{M_{\epsilon}}{1-M_{\epsilon}} s_{r}^{\circ} \frac{M_{\epsilon}}{1-M_{\epsilon}} s_{a}^{\circ}+\frac{M_{\epsilon}}{1-M_{\epsilon}} s_{r a}^{\circ}\right],
$$

where $s_{r}^{\circ}, s_{a}^{\circ}$ and $s_{r a}^{\circ}$ respectively denote the derivatives of $s^{\circ}$ with respect to $j_{r}, j_{a}$, and $j_{r}$ and $j_{a}$, all evaluated at $j_{r}=j_{a}=0$. If these derivatives are calculated using the parallel convention for $J_{a}$ and $J_{r}$, the diffusion $(d d)$ derivatives take the form

$$
\delta \xi_{\left[\alpha, \alpha^{\prime}\right],(d d)}^{(2) D}=\delta_{\alpha, \alpha^{\prime}}\left(\frac{M_{\epsilon}}{1-M_{\epsilon}}\right)_{\alpha \alpha}+\left(\frac{M_{\epsilon}}{1-M_{\epsilon}}\right)_{\alpha \alpha^{\prime}}\left(\frac{M_{\epsilon}}{1-M_{\epsilon}}\right)_{\alpha^{\prime} \alpha},
$$

and the cooperon $(d d)$ derivatives is given by the same formula with $\hat{\alpha}$ in place of $\alpha$. Notice that this expression agrees with the derivatives 124) and 125) of the diagonal approximation to the trace formula for the generating function in Section 4.2. If the derivatives of $s^{\circ}$ found with the crossed convention are plugged into 221 , one gets the diffusion $(d d)$ derivatives

$$
\delta \xi_{\left[\alpha, \alpha^{\prime}\right],(d d)}^{\times(2) D} \equiv\left(\frac{M_{\epsilon}}{1-M_{\epsilon}}\right)_{\alpha \alpha^{\prime}}+\delta_{\alpha, \alpha^{\prime}}\left(\frac{M_{\epsilon}}{1-M_{\epsilon}}\right)_{\alpha \alpha}\left(\frac{M_{\epsilon}}{1-M_{\epsilon}}\right)_{\alpha \alpha}
$$

and the corresponding expression with $\hat{\alpha}$ in place of $\alpha$ for the cooperon $(d d)$ derivatives. This is again the result obtained in (124) and (125) for the derivatives of the diagonal approximation to the generating function. The derivatives of the second order mean field generating function 216 have to be removed from the previous formulae. They read

$$
\delta \xi_{\left[\alpha, \alpha^{\prime}\right]}^{\mathrm{MF}(2)}=\left(\frac{1}{1-e^{-2 \epsilon}}\right)^{2} \sigma_{r} \sigma_{a}+\left(\frac{1}{1-e^{-2 \epsilon}}\right) \sigma_{r a},
$$

where the indices $r$ and $a$ denote the derivatives taken on $\sigma$, which are all evaluated at the origin. These derivatives can be calculated according to the parallel or the crossed conventions. The results obtained are given by the formulae (222) and (223) by systematically replacing the sum of classical walks $M_{\epsilon}\left(1-M_{\epsilon}\right)^{-1}$ with the uniform component of $\left(1-M_{\epsilon}\right)^{-1}$, which is defined as in (35) and reads $\left(1-e^{-2 \epsilon}\right)^{-1}|1\rangle\langle 1|$. This draws a parallel between the zero mode and the uniform component. Finally, when the formula 219) is applied, the terms in 222 and 223 that are too singular in $\epsilon$ are exactly compensated by the mean field derivatives, and one is left with the finite result

$$
C_{\alpha, \alpha^{\prime},(d d)}^{G, D}=\frac{R_{\alpha \alpha^{\prime}}+R_{\alpha^{\prime} \alpha}}{(2 B)^{2}}-\frac{2}{(2 B)^{3}} \quad \text { and } \quad C_{\alpha \alpha^{\prime},(d d)}^{\times G, D}=\delta_{\alpha, \alpha^{\prime}} C_{\alpha \alpha^{\prime},(d d)}^{G, D}
$$


for the parallel and crossed diffusion $(d d)$ Gaussian intensity correlation matrix. In these formulae, the matrix $R$ denotes the massive component defined by the decomposition (35). The cooperon contributions to the Gaussian intensity correlation matrix read

$$
C_{\alpha \alpha^{\prime},(d d)}^{G, C}=\frac{R_{\hat{\alpha} \alpha^{\prime}}+R_{\alpha^{\prime} \hat{\alpha}}}{(2 B)^{2}}-\frac{2}{(2 B)^{3}} \quad \text { and } \quad C_{\alpha \alpha^{\prime},(d d)}^{\times G, C}=\delta_{\alpha, \alpha^{\prime}} C_{\alpha \alpha^{\prime},(d d)}^{G, C}
$$

The surprising second terms of the formulae in (225) and 226) originate from the fact that the zero mode contribution to 222) and (223) is the uniform component of $\left(1-M_{\epsilon}\right)^{-1}$ and not $M_{\epsilon}\left(1-M_{\epsilon}\right)^{-1}$. This discrepancy is due to the additional symmetry $\tilde{Y}=Y^{\tau}$ of the zero mode. Notice however that these second terms are of higher order in $(2 B)^{-1}$, and are thus of minor importance when the large graph limit is considered.

Using the same strategy as above, it is not difficult to calculate more retarded derivatives in (220) and to remove the mean field contributions. If $[\alpha]$ is a list of $q \geq 2$ directed bonds, and if the convention $\sigma=\mathrm{id} \in S_{q}$ for the generating function 212 is chosen, one gets the formula

$$
C_{[\alpha],(d d)}^{G, D}=\frac{1}{(q-1)(2 B)^{q}} \sum_{\substack{k, l=0 \\ k \neq l}}^{q-1} R_{\alpha_{k} \alpha_{l}}-\frac{q}{(2 B)^{q+1}}
$$

for the diffusion $(d d)$ Gaussian autocorrelation function of degree $q$, and the same formula with $\hat{\alpha}_{0}$ in place of $\alpha_{0}$ for the cooperon $(d d)$ Gaussian autocorrelation function of degree $q$.

\subsection{Off-Diagonal Modes in Direction Space}

Let us now investigate the full second order generating functions taking into account the modes $Z$ and $\tilde{Z}$ that are off-diagonal in direction space. The parametrizations (200) of $Z$ and $\tilde{Z}$ in time-reversal space can be kept, and hence the formulae (203) and (204) also hold in the presence of off-diagonal modes. This implies in particular that the second order generating functions factorize into diffusion and cooperon generating functions as in (205), and that the cooperon formulae, which are considered only if time-reversal invariance is conserved, can be found from their diffusion counterparts by replacing $J_{a}\left(j_{a}\right)$ with $J_{a}\left(j_{a}\right)^{\mathcal{T}}$. One can thus temporarily concentrate on the diffusion modes only.

One can distinguish between diagonal and off-diagonal modes and introduce the notations

$$
Z_{\beta}^{\text {diag }} \equiv Z_{\beta \beta} \quad \text { and } \quad Z_{\beta}^{\text {off }} \equiv Z_{\beta \hat{\beta}}
$$

and similarly for $\tilde{Z}$. The quadratic action couples diagonal modes with themselves, which is precisely the part treated in the previous subsection, off-diagonal modes with themselves, and diagonal modes with off-diagonal modes.

The integration scheme used here is similar to the one that leads to the explicit formula (212) for the $(d d)$ Gaussian generating functions in terms of four determinants. Let us first focus on the commuting components $Z_{j s s}, s \in\{B, F\}$, of the fields. The row and column vectors defined in 207), whose purpose is to write the diagonal action 
$S_{(d d)}^{(2) D}$ as a quadratic form, are adapted to the situation where the fields $Z$ and $\tilde{Z}$ are as in (228). Let us define

$$
\tilde{w}_{\beta}=\left(\tilde{z}_{1 \overline{0}}^{\text {diag }}, z_{4 \overline{0}}^{\dagger \text { †iag }}, \tilde{z}_{1 \overline{0}}^{\text {off }}, z_{4 \overline{0}}^{\dagger \text { off }}\right)_{\beta}
$$

where $\tilde{z}_{1 \overline{0}}^{\text {diag }}$ and $z_{4 \overline{0}}^{\dagger \text { diag }}$ are formed with the diagonal modes of $\tilde{z}_{1 \overline{0}}$ and $z_{4 \overline{0}}^{\dagger}$ defined in 207), and $\tilde{z}_{1 \overline{0}}^{\text {off }}$ and $z_{4 \overline{0}}^{\dagger \text { toff }}$ are formed with the off-diagonal ones. We proceed in the same way with the column vectors and introduce

$$
w_{\beta}=\left(z_{1 \overline{0}}^{\operatorname{diag} T}, \tilde{z}_{4 \overline{0} \beta}^{\dagger \text { diag } T}, z_{1 \overline{0}}^{\text {off } T}, \tilde{z}_{4 \overline{0} \beta}^{\dagger \text { off } T}\right)_{\beta}^{T} .
$$

Then, a careful inspection of the diffusion second order action (203) and some algebra show that the part of this action involving the commuting variables is the quadratic form

$$
S_{\overline{0}}^{(2) D}=\sum_{\beta, \beta^{\prime}=1}^{2 B} \tilde{w}_{\beta} \mathcal{B}_{\beta \beta^{\prime}} w_{\beta^{\prime}}
$$

defined by the $16 B \times 16 B$ matrix

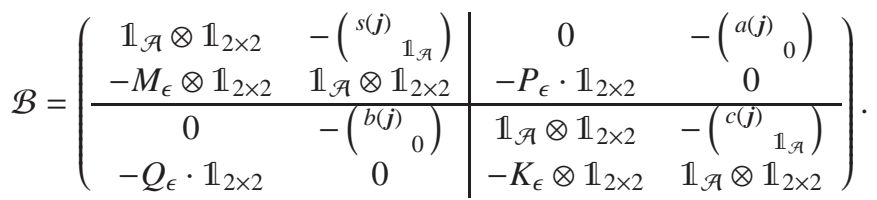

Notice that the block coupling the diagonal modes together is precisely 210). In the quadratic form (232), the $2 B \times 2 B$ matrices $P_{\epsilon}, Q_{\epsilon}$ and $K_{\epsilon}$ are defined by

$$
P_{\epsilon \beta \beta^{\prime}} \equiv S_{\epsilon \beta \beta^{\prime}} S_{\epsilon \beta \hat{\beta}^{\prime}}^{*}, \quad Q_{\epsilon \beta \beta^{\prime}} \equiv S_{\epsilon \beta \beta^{\prime}} S_{\epsilon \hat{\beta} \beta^{\prime}}^{*} \quad \text { and } \quad K_{\epsilon \beta \beta^{\prime}} \equiv \delta_{\hat{\beta}, \beta^{\prime}} S_{\epsilon \beta \hat{\beta}} S_{\epsilon \hat{\beta} \beta^{\prime}}^{*}
$$

In fact, $P_{\epsilon}$ and $Q_{\epsilon}$ both vanish since we only consider simple graphs. The square of $K \equiv \lim _{\epsilon \rightarrow 0} K_{\epsilon}$ is the diagonal matrix

$$
\left(K^{2}\right)_{\beta \beta^{\prime}}=\delta_{\beta, \beta^{\prime}} M_{\beta \hat{\beta}} M_{\hat{\beta} \beta}
$$

which only depends on $S$ through the classical map $M$. It can be deduced from (234) that the spectrum of $K$ is real and is contained in $(-1,1)$ if the graph is ergodic. In (232), $s(\boldsymbol{j})$ is the matrix defined in 209,,$c(\boldsymbol{j})$ is another matrix satisfying $c(\mathbf{0})=1$, and $a(\boldsymbol{j})$ and $b(\boldsymbol{j})$ are given by

$$
a\left(j_{a}, \boldsymbol{j}_{\boldsymbol{r}}\right)_{\beta \beta^{\prime}} \equiv \delta_{\beta \beta^{\prime}} j_{a} E_{\hat{\beta} \beta}^{(a)}+\delta_{\beta \hat{\beta}^{\prime}}\left(\boldsymbol{j}_{\boldsymbol{r}} \boldsymbol{E}^{(\boldsymbol{r})}\right)_{\beta \hat{\beta}}
$$

and

$$
b\left(j_{a}, \boldsymbol{j}_{r}\right)_{\beta \beta^{\prime}} \equiv \delta_{\beta \beta^{\prime}} j_{a} E_{\beta \hat{\beta}}^{(a)}+\delta_{\beta \hat{\beta}^{\prime}}\left(\boldsymbol{j}_{\boldsymbol{r}} \boldsymbol{E}^{(\boldsymbol{r})}\right)_{\beta \hat{\beta}} .
$$

It can be checked that $a(\boldsymbol{j})$ and $b(\boldsymbol{j})$ both vanish if the convention $\sigma=$ id for the generating functions is used. 
The determinant of $\mathcal{B}$ in (232) can be calculated using the rule (134) adapted to conventional determinants. The result is the product

$$
\operatorname{det} \mathcal{B}=\Delta_{F F-F F} \cdot \Delta_{B B-B B}(\boldsymbol{j})
$$

where $\Delta_{F F-F F} \equiv \Delta_{B B-B B}(\mathbf{0})$,

$$
\Delta_{B B-B B}(\boldsymbol{j})=\operatorname{det}\left(\mathbb{1}_{\mathcal{A}}-s(\boldsymbol{j}) M_{\epsilon}\right) \operatorname{det}\left(\mathbb{1}_{\mathcal{A}}-c(\boldsymbol{j}) K_{\epsilon}\right) \operatorname{det}\left(\mathbb{1}_{\mathcal{A}}-N_{\epsilon}(\boldsymbol{j})\right),
$$

and

$$
N_{\epsilon}(\boldsymbol{j}) \equiv M_{\epsilon} \frac{1}{1-s(\boldsymbol{j}) M_{\epsilon}} a(\boldsymbol{j}) K_{\epsilon} \frac{1}{1-c(\boldsymbol{j}) K_{\epsilon}} b(\boldsymbol{j}) .
$$

The first factor in 237) comes from the couplings between $F F$ and $F F$ components of the variables in the vectors $\tilde{w}_{\beta}$ and $w_{\beta}$. The second factor comes from the couplings between $B B$ and $B B$ components. The fact that the contribution of the $F F-F F$ couplings can be found from the contribution of the $B B-B B$ couplings by setting all the sources to zero can actually already be observed on the formula (203) for the diffusion second order action $S^{(2) D}$. A further look at this formula enables one to deduce the contributions of the couplings between the anticommuting variables. It can be seen that the matrix mixing the $B F$ components of the row vectors $\tilde{w}_{\beta}$ and the $F B$ components of column vectors $w_{\beta}$ has determinant $\Delta_{B B-B B}\left(j_{a}, \mathbf{0}\right)$, and similarly, the matrix mixing the $F B$ components of the row vectors $\tilde{w}_{\beta}$ and the $B F$ components of the column vectors $w_{\beta}$ has determinant $\Delta_{B B-B B}\left(0, \boldsymbol{j}_{\boldsymbol{r}}\right)$. Hence, the diffusion second order generating function reads

$$
\xi_{[\alpha]}^{(2) D}=\xi_{[\alpha],(d d)}^{(2) D} \cdot \xi_{[\alpha],(o o)}^{(2) D} \cdot \xi_{[\alpha],(d o)}^{(2) D},
$$

where the first factor in the right-hand side is the diffusion $(d d)$ second order generating function (212),

$$
\xi_{[\alpha],(o o)}^{(2) D}\left(j_{a}, j_{\boldsymbol{r}}\right) \equiv \frac{\operatorname{det}\left(\mathbb{1}_{\mathcal{A}}-c\left(j_{a}, \mathbf{0}\right) K_{\epsilon}\right) \operatorname{det}\left(\mathbb{1}_{\mathcal{A}}-c\left(0, \boldsymbol{j}_{\boldsymbol{r}}\right) K_{\epsilon}\right)}{\operatorname{det}\left(\mathbb{1}_{\mathcal{A}}-K_{\epsilon}\right) \operatorname{det}\left(\mathbb{1}_{\mathcal{A}}-c\left(j_{a}, \boldsymbol{j}_{\boldsymbol{r}}\right) K_{\epsilon}\right)},
$$

and

$$
\xi_{[\alpha],(d o)}^{(2) D}\left(j_{a}, \boldsymbol{j}_{\boldsymbol{r}}\right) \equiv \frac{\operatorname{det}\left(\mathbb{1}_{\mathcal{A}}-N_{\epsilon}\left(j_{a}, \mathbf{0}\right)\right) \operatorname{det}\left(\mathbb{1}_{\mathcal{A}}-N_{\epsilon}\left(0, \boldsymbol{j}_{\boldsymbol{r}}\right)\right)}{\operatorname{det}\left(\mathbb{1}_{\mathcal{A}}-N_{\epsilon}(0, \mathbf{0})\right) \operatorname{det}\left(\mathbb{1}_{\mathcal{A}}-N_{\epsilon}\left(j_{a}, \boldsymbol{j}_{\boldsymbol{r}}\right)\right)} .
$$

These functions all have the property that they become identically one in a neighborhood of the origin if either the advanced or the retarded derivatives are set to zero. Hence, their product (240), and the cooperon analogs, share the same property. It follows that the derivatives of these functions satisfy

$$
\delta \xi_{[\alpha]}^{(2)}=\sum_{x \in\{d d, o o, d o\}} \delta \xi_{[\alpha],(x)}^{(2) D}+(\kappa-1) \sum_{x \in\{d d, o o, d o\}} \delta \xi_{[\alpha],(x)}^{(2) C} .
$$

Moreover, since $K$ has no eigenvalue unity, the (do) generating functions at $\epsilon=0$ are analytic in a neighborhood of the origin, and hence, their derivatives cannot contribute to the Gaussian autocorrelation functions [198]. 
If the convention $\sigma=\mathrm{id}$ is used for the generating functions, then the functions $a$ and $b$ in (235) and 236) vanish. In this case, the (do) generating function (242) is equal to one, its derivatives vanish, and only the $(d d)$ derivatives remain in (243). Therefore, from (243), 227) and this remark, the Gaussian autocorrelation functions (198) of degree $q$ read

$$
C_{[\alpha]}^{G}=C_{[\alpha],(d d)}^{G}=\frac{1}{(q-1)(2 B)^{q}} \sum_{\substack{k, l=0 \\ k \neq l}}^{q-1} R_{\alpha_{k} \alpha_{l}}-\frac{q}{(2 B)^{q+1}}+(\kappa-1)\left\{\alpha_{0} \rightarrow \hat{\alpha}_{0}\right\} .
$$

Here, the last term $\left\{\alpha_{0} \rightarrow \hat{\alpha}_{0}\right\}$ stands for the right-hand side with $\alpha_{0}$ replaced with $\hat{\alpha}_{0}$. In summary, the off-diagonal modes do not bring any additional contribution to the Gaussian autocorrelation functions if the convention $\sigma=$ id is used.

Let us now consider the situation where $q=2,[\alpha]=\left[\alpha, \alpha^{\prime}\right]$, and the generating function is defined with the crossed convention. In this case, the two derivatives on the (do) generating function (242) give

$$
\delta \xi_{\left[\alpha, \alpha^{\prime}\right],(d o)}^{(2)}=\operatorname{tr}\left[\frac{1}{1-N_{\epsilon}} N_{\epsilon, 0} \frac{1}{1-N_{\epsilon}} N_{\epsilon, 1}+\frac{1}{1-N_{\epsilon}} N_{\epsilon, 01}\right],
$$

where $N_{\epsilon}$ denotes the value of the function $N_{\epsilon}\left(j_{0}, j_{1}\right)$ at the origin, and $N_{\epsilon, 0}, N_{\epsilon, 1}$ and $N_{\epsilon, 01}$ stand for its derivatives at the origin. Since $a$ and $b$ are zero at the origin, $N_{\epsilon}, N_{\epsilon, 0}$ and $N_{\epsilon, 1}$ vanish. Therefore, only the second term in the trace of (245) contributes, and a short calculation shows that the diffusion (do) Gaussian approximation to the intensity correlation matrix in the crossed convention reads

$$
C_{\alpha \alpha^{\prime},(d o)}^{\times G, D}=\lim _{\epsilon \rightarrow 0} \frac{\epsilon}{B} \delta_{\alpha^{\prime}, \hat{\alpha}}\left(\frac{M_{\epsilon}}{1-M_{\epsilon}}\right)_{\alpha \hat{\alpha}}\left[\left(\frac{K}{1-K}\right)_{\alpha \alpha}+\left(\frac{K}{1-K}\right)_{\hat{\alpha} \hat{\alpha}}\right]
$$

The cooperon result turns out to be the same. With the decomposition 35) of the classical walks, and using the fact that the diagonal elements of $K^{n}$ vanish if the integer $n$ is odd, one gets

$$
C_{\alpha \alpha^{\prime},(d o)}^{\times G}=\kappa \frac{\delta_{\alpha, \hat{\alpha}^{\prime}}}{(2 B)^{2}}\left[R_{\alpha \alpha}^{K}+R_{\hat{\alpha} \hat{\alpha}}^{K}\right],
$$

where

$$
R_{\alpha \alpha^{\prime}}^{K} \equiv\left(\frac{K^{2}}{1-K^{2}}\right)_{\alpha \alpha^{\prime}}=\delta_{\alpha, \alpha^{\prime}} \frac{M_{\alpha \hat{\alpha}} M_{\hat{\alpha} \alpha}}{1-M_{\alpha \hat{\alpha}} M_{\hat{\alpha} \alpha}} .
$$

The matrix $R^{K}$, called the back-scattering matrix, is formed with all the oriented walks followed with the classical map $M$ which involve only back-scatterings $\beta \rightarrow \hat{\beta}$ and no transmission. Together with (225) and (226), 247) yields the Gaussian contribution

$$
\begin{aligned}
C_{\alpha \alpha^{\prime}}^{\times G}= & \frac{\delta_{\alpha, \alpha^{\prime}}}{(2 B)^{2}}\left[2 R_{\alpha \alpha}+(\kappa-1)\left(R_{\hat{\alpha} \alpha}+R_{\alpha \hat{\alpha}}\right)\right]-\delta_{\alpha, \alpha^{\prime}} \frac{2 \kappa}{(2 B)^{3}} \\
& +\kappa \frac{\delta_{\alpha, \hat{\alpha}^{\prime}}}{(2 B)^{2}}\left[R_{\alpha \alpha}^{K}+R_{\hat{\alpha} \hat{\alpha}}^{K}\right]
\end{aligned}
$$

to the intensity correlation matrix in the crossed convention.

For $q=2$ and $\alpha=\alpha^{\prime}$, the parallel and crossed results (244) and (249) coincide. Notice that in this case, the parallel and crossed sums over oriented walks represented in Figure 2 are also the same. 


\section{Criteria and Rates of Universality}

\subsection{Full Universality and Criterion for Ergodicity}

The calculation scheme summarized in (193) leads to truncated autocorrelation functions, which are sums of the mean field contributions obtained in Section 5 and the Gaussian contributions obtained in Section 6 . On one hand, the mean field results coincide with the universal Gaussian Random Wave Models introduced in Subsection 3.2. On the other hand, the Gaussian quantities in (244) depend on the quantum graph, but do so only through its classical map $M$, and more precisely through its matrix $R$, which is defined in (35) and represents the massive component of the sum of classical paths $\frac{M_{\epsilon}}{1-M_{\epsilon}}$. The importance of the truncated autocorrelation functions is twofold. Firstly, their Gaussian contributions can be compared with their universal mean field parts in the limit of large graphs. These comparisons lead to conditions on the increasing sequence of quantum graphs $\left\{\left(G_{l}, S_{l}\right)\right\}_{l \in \mathbb{N}}$ to asymptotically follow the predictions of the universal Gaussian Random Wave Models, in which case we say that full universality is met. Secondly, for a class of increasing sequences larger than this universal class, the truncated quantities approximate the exact autocorrelation functions (42).

Let $(G, S)$ be an ergodic simple quantum graph, and let $[\alpha]$ be a list of $q$ directed bonds for some integer $q \geq 2$. The mean field autocorrelation functions found in Subsection 5.2 and the Gaussian autocorrelation functions in (244) lead to the truncated autocorrelation functions

$$
\tilde{C}_{[\alpha]}=\frac{c(\kappa,[\alpha])}{(2 B)^{q}}+\frac{1}{(q-1)(2 B)^{q}} \sum_{\substack{k, l=0 \\ k \neq l}}^{q-1}\left[R_{\alpha_{k} \alpha_{l}}+(\kappa-1)\left\{\alpha_{0} \rightarrow \hat{\alpha}_{0}\right\}\right]-\frac{\kappa q}{(2 B)^{q+1}} .
$$

The parameter $\kappa$ is equal to one or two depending whether time-reversal symmetry is broken or conserved, and $c(\kappa,[\alpha])$ is the combinatorial factor defined in the following way. Suppose that each directed bond $\beta$ appears exactly $q_{\beta}$ times in the list $[\alpha]$, then $c(1,[\alpha]) \equiv \prod_{\beta=1}^{2 B} q_{\beta}$ !. Suppose now that $p_{b}$ denotes the number of directed bonds in the list $[\alpha]$ supported on bond $b$, then $c(2,[\alpha]) \equiv \prod_{b=1}^{B} p_{b}$ !. The intensity correlation matrix and the moments implied by (250) read

$$
\tilde{C}_{\alpha \alpha^{\prime}}=\frac{1}{4 B^{2}}\left(1+\delta_{\alpha \alpha^{\prime}}+R_{\alpha \alpha^{\prime}}+R_{\alpha^{\prime} \alpha}\right)+\frac{\kappa-1}{4 B^{2}}\left(\delta_{\alpha \hat{\alpha}^{\prime}}+R_{\alpha \hat{\alpha}^{\prime}}+R_{\hat{\alpha}^{\prime} \alpha}\right)
$$

and

$$
\tilde{M}_{q, \alpha}=\frac{q !}{(2 B)^{q}}\left(1+\frac{1}{(q-1) !} R_{\alpha \alpha}+\frac{\kappa-1}{q !}\left(R_{\alpha \hat{\alpha}}+R_{\hat{\alpha} \alpha}+(q-2) R_{\alpha \alpha}\right)\right) .
$$

In these two expressions, the last term of (250), which is of higher order in the inverse number of bonds $B^{-1}$, has been neglected since we are ultimately interested in the large graph limit $B \rightarrow \infty$.

For graphs in the orthogonal class, the exact autocorrelation functions $C_{[\alpha]}$ in (42) do reflect the symmetry $\left|a_{\alpha}\right|^{2}=\left|a_{\hat{\alpha}}\right|^{2}$ of the wave function intensities. The intensity correlation matrix 251) indeed satisfies $\tilde{C}_{\alpha \alpha^{\prime}}=\tilde{C}_{\hat{\alpha} \alpha^{\prime}}$ if $\kappa=2$, but the truncated autocorrelation functions (252) of degree $q \geq 3$ do not obey such a symmetry in general. A 
comparison of 251) with a numerically obtained intensity correlation matrix for complete quantum graphs with various choices of scattering matrices reveals that (251) captures the asymptotics $B \rightarrow \infty$ very well at least as long as the intensity matrix is not dominated by the massive contributions (see Figure 3). A numerical comparison of higher moments to 252 (not shown) reveals that their massive contributions are not as well approximated by our theory. We should however emphasize that the numerical evaluation of higher moments is not very stable. The statements we put forward below are all consistent with the numerically obtained data we have.

Let $\left\{\left(G_{l}, S_{l}\right)\right\}_{l \in \mathbb{N}}$ be a sequence of increasing ergodic simple graphs that are either all in the unitary or in the orthogonal class. The formula (250) suggests that the mean field term is the dominant one if and only if the sequence of matrices $\left\{R_{l}\right\}_{l \in \mathbb{N}}$ converges to zero as $l \rightarrow \infty$. Consequently, we conjecture that the Gaussian Random Wave Models (48) or (53) are asymptotically met in the sequence $\left\{\left(G_{l}, S_{l}\right)\right\}_{l \in \mathbb{N}}$ if and only if the norm of the matrices $R_{l}$ decay as $l \rightarrow \infty$. Note that $R_{l} \rightarrow 0$ introduces a small parameter in which a systematic expansion may be performed. We strongly believe that our approach may be extended to a rigorous proof that $R_{l} \rightarrow 0$ implies convergence to the Gaussian Random Wave Model.

Notice that, by the definition (35) of the matrix $R_{\epsilon}$, the decay of the sequence $R_{\epsilon, l}$ is equivalent to

$$
\lim _{l \rightarrow \infty} \frac{M_{\epsilon, l}}{1-M_{\epsilon, l}}=O(\epsilon) .
$$

This is also equivalent to the decay of $R_{l} \equiv \lim _{\epsilon \rightarrow 0} R_{\epsilon, l}$. Since all the components of the matrix $M_{\epsilon, l} \equiv e^{-2 \epsilon} M_{l}$ are non-negative, 253) implies that

$$
\lim _{l \rightarrow \infty} M_{l}=0 .
$$

A necessary condition for this property to occur, and hence for full universality to be met, is that the valencies of the vertices all tend to infinity. This condition has already been derived at the end of Subsection 3.2, where the Random Wave Models are built. Conversely, suppose that 254) is fulfilled, then the equality (253) also holds, and thus $R_{l}$ decays. Therefore, the necessary and sufficient condition for full universality that is conjectured above is actually equivalent to (254).

The expression (251), together with the formula [69], generate a truncated expression $\tilde{\mathcal{F}}_{V}$ for the fluctuations of an observable $V$. The asymptotic quantum ergodicity problem described in Subsection 3.3 can be addressed in terms of these truncated fluctuations. Moreover, in the situations where $\tilde{\mathcal{F}}_{V}$ decays, $\tilde{\mathcal{F}}_{V}$ is expected to approximate the exact fluctuations $\mathcal{F}_{V}$. A direct calculation shows that, for an observable $V$ with $\bar{V}=0$,

$$
\tilde{\mathcal{F}}_{V}=\kappa \frac{\operatorname{tr}(V L)^{2}}{(\operatorname{tr} L)^{2}}+2 \kappa \frac{\sum_{\beta, \beta^{\prime}}[V L \cdot R \cdot V L]_{\beta \beta^{\prime}}}{(\operatorname{tr} L)^{2}}
$$

This formula motivates the following criterion for asymptotic quantum ergodicity to be met in an increasing sequence of quantum graphs. We conjecture that an increasing sequence $\left\{\left(G_{l}, S_{l}\right)\right\}_{l \in \mathbb{N}}$ of ergodic simple graphs is asymptotically quantum ergodic if and only if

$$
\lim _{l \rightarrow \infty} \frac{\sum_{\beta, \beta^{\prime}}\left[V_{l} L_{l} \cdot R_{l} \cdot V_{l} L_{l}\right]_{\beta \beta^{\prime}}}{\left(\operatorname{tr} L_{l}\right)^{2}}=0
$$



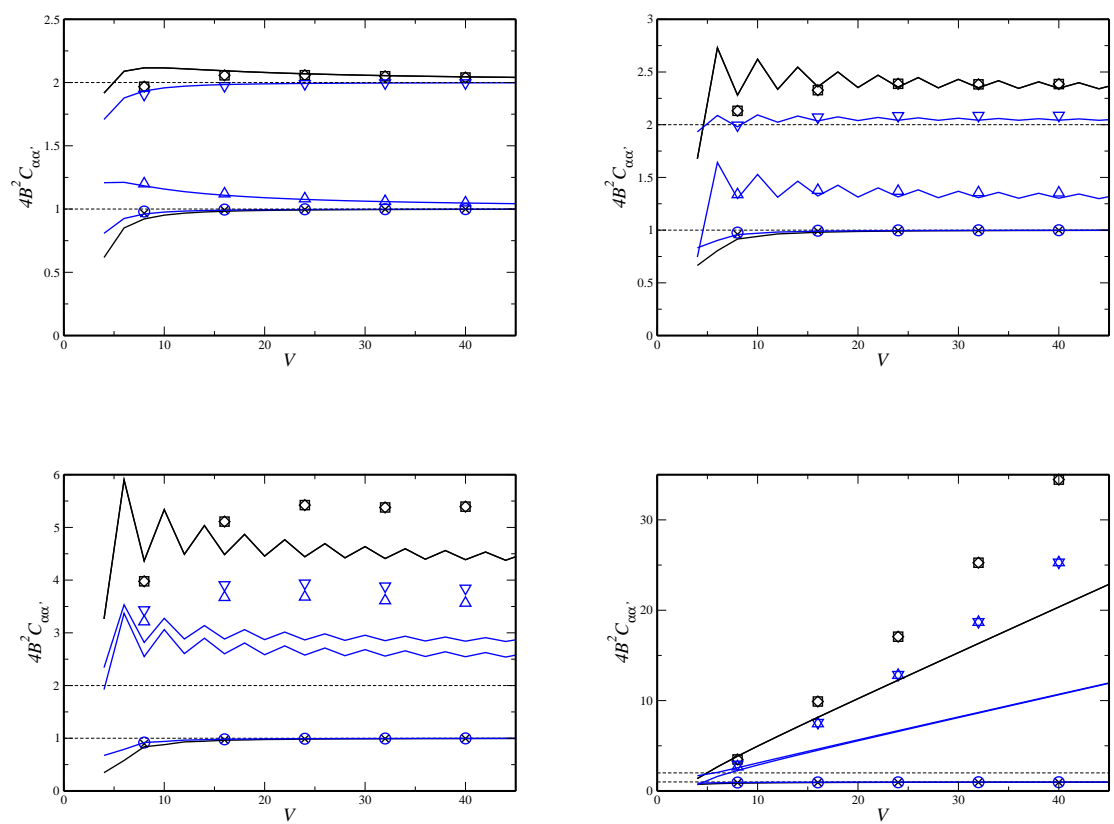

Figure 3: Rescaled matrix elements of the intensity correlation matrix for complete graphs with $V$ vertices.

The four panels correspond to four different choices of the scattering matrix. The upper left panel is a complete DFT graph. For the upper right and lower left panel the scattering matrix that corresponds to one vertex has been chosen by the following unitary transformation of a DFT matrix $\sigma^{i}(\lambda)=e^{-i \pi / 4} \frac{1-\lambda+e^{i \pi / 4}(1+\lambda) \sigma^{\mathrm{DFT}}}{1+\lambda+e^{i \pi / 4}(1-\lambda) \sigma^{\mathrm{DFT}}}$ with the values $\lambda=0.5$ for the upper right panel and $\lambda=0.25$ in the lower left panel. For the lower right panel Neumann scattering matrices have been chosen.

An additional magnetic field was applied to break time-reversal symmetry. Black symbols and lines correspond to results for the orthogonal class and blue symbols and lines to results for the unitary class where the symbols correspond to numerically obtained intensity correlations and full lines to the corresponding prediction (251). The squares (orthogonal) and downwards pointing triangles (unitary class) give the average rescaled diagonal element $2 B \sum_{\alpha=1}^{2 B} C_{\alpha \alpha}$ (the Gaussian Random Wave Model predicts the value 2 indicated by the upper dashed line). The diamonds (orthogonal) and upwards pointing triangles (unitary class) give the average rescaled time-reversed diagonal element $2 B \sum_{\alpha=1}^{2 B} C_{\alpha \hat{\alpha}}$ (the Gaussian Random Wave Model predicts the values 1 in the unitary and 2 in the orthogonal class). Note that the diamonds and squares always lie on top of each other. Eventually, crosses (orthogonal) and circles (unitary class) give the average rescaled element $\frac{B}{B-4} \sum_{\alpha^{\prime}, \alpha=1: \alpha \neq \alpha^{\prime}, \alpha \neq \hat{\alpha}^{\prime}}^{2 B} C_{\alpha \alpha^{\prime}}$ (the Gaussian Random Wave Model predicts the value 1). The corresponding predictions from (251) are given by the full lines. 
for any acceptable sequence $\left\{V_{l}\right\}_{l \in \mathbb{N}}$ with $\bar{V}_{l}=0$. We will give a slightly more detailed variant of this conjecture (and a discussion of possible obstruction to its validity) below.

Moreover, if the stronger condition $R_{l} \rightarrow 0$ is fulfilled, the increasing sequence of graphs is fully universal, and the convergence rate of $\tilde{\mathcal{F}}_{V}$, and hence of $\mathcal{F}_{V}$, is then also universal. As in the case of the validity of the Gaussian Random Wave Model we strongly believe that our approach can be extended to a rigorous proof using $R$ (or an equivalent quantity) as the small parameter.

Note that the crossed formulae for the massive (Gaussian) contribution to the intensity correlation matrix in 249) differ from the formulae in 244), which are used above. The crossed expressions only involve the diagonal components $(\alpha, \alpha)$ and the components $(\alpha, \hat{\alpha})$ of the matrix $R$ - they also contain a new backscattering term. These do not obey $C_{\alpha \alpha}=C_{\alpha \hat{\alpha}}$ and, indeed, they do not capture the massive corrections in the exact correlation matrix as well as the parallel convention in a numerical test (Figure 3 only presents the results for the parallel convention).

Let us now consider observables $V$ such that $V_{b} L_{b}=\frac{\operatorname{tr} L}{2 B}$ on half of the bonds, and $V_{b} L_{b}=-\frac{\operatorname{tr} L}{2 B}$ on the other half. The set of such observables is actually sufficiently large to compare the intensities of the wave function on the different bonds. Moreover, they provide acceptable sequences, according to 63). For such observables, 255 yields

$$
\tilde{\mathcal{F}}_{V} \approx \kappa \frac{1}{2 B}+\frac{2 \kappa \operatorname{tr}\left(R+R \sigma_{1}^{d}\right)}{(2 B)^{2}},
$$

where we neglected almost all off-diagonal terms (apart from those obeying $\alpha^{\prime}=\hat{\alpha}$ ) in the double sum in 255 . An increasing sequence $\left\{\left(G_{l}, S_{l}\right)\right\}_{l \in \mathbb{N}}$ of simple graphs is then expected to be asymptotically quantum ergodic if and only if both

$$
\lim _{l \rightarrow \infty} \frac{\operatorname{tr} R_{l}}{\left(2 B_{l}\right)^{2}}=\lim _{l \rightarrow \infty} \frac{1}{\left(2 B_{l}\right)^{2}} \sum_{i=2}^{2 B_{l}} \frac{1-m_{l, i}}{m_{l, i}}=0
$$

and

$$
\lim _{l \rightarrow \infty} \frac{\operatorname{tr} R_{l} \sigma_{1}^{d}}{(2 B)^{2}}=0
$$

hold. In (258), the complex numbers $m_{l, i}, 2 \leq i \leq 2 B$, are the $2 B-1$ non-zero masses, that is the $2 B-1$ eigenvalues of the matrix $1-M_{l}$.

\subsection{Quantum Ergodicity and the Classical Spectral Gap}

Sufficient conditions for the condition (258) to be fulfilled or violated in an increasing sequence $\left\{\left(G_{l}, S_{l}\right)\right\}_{l \in \mathbb{N}}$ of ergodic simple graphs can be given in terms of the sequence $\left\{\Delta_{M_{l}}\right\}_{l \in \mathbb{N}}$ of spectral gaps of $1-M_{l}$.

Let us first consider the case that all non-zero masses stay away from the origin. The sum in 258 behaves like $2 B_{l}$, and hence, after dividing by $\left(2 B_{l}\right)^{2}$, the large graphs limit vanishes, and 258) (and similarly 259) holds.

Now let us turn to the case that some masses approach zero as $B_{l} \rightarrow \infty$. For sake of simplicity, the index $l$ of the quantum graph $\left(G_{l}, S_{l}\right)$ will be dropped. Let us order the spectrum $\left\{m_{i}\right\}_{i \in \mathbb{N}_{2 B}}$ of $1-M$ such that $\left|m_{i}\right| \leq\left|m_{i+1}\right|$ for all $i \in \mathbb{N}_{2 B}$, and let us now 
suppose that the spectral gap $\Delta_{M} \equiv\left|m_{2}\right|$ approaches the origin with an exponential rate $\alpha>0$, that is

$$
\left|m_{2}\right| \sim(2 B)^{-\alpha} \text {. }
$$

The matrix $R$ is real since $M$ is real and the vector $|1\rangle$ is also real. It follows that the massive contribution of the fluctuations (257) can be written

$$
\tilde{\mathcal{F}}_{V}^{M} \equiv \frac{2 \kappa}{(2 B)^{2}} \sum_{i=2}^{2 B} \mathfrak{R} \frac{1-m_{i}}{m_{i}}=\frac{2 \kappa}{(2 B)^{2}} \sum_{i=2}^{2 B} \frac{\mathfrak{R} m_{i}}{\left|m_{i}\right|^{2}}-\frac{2 \kappa(2 B-1)}{(2 B)^{2}} .
$$

The second term of the right-hand side behaves like $(2 B)^{-1}$, so that $(258)$ is satisfied if and only if the first term of the right-hand side, denoted by $\hat{\mathcal{F}}_{V}^{M}$ in what follows, decays. With the obvious inequality $\mathfrak{R} m_{i} \leq\left|m_{i}\right|$, one gets

$$
\hat{\mathcal{F}}_{V}^{M} \leq \frac{2 \kappa}{(2 B)^{2}} \sum_{i=2}^{2 B} \frac{1}{\left|m_{i}\right|} \leq \frac{2 \kappa}{(2 B)^{2}} \frac{2 B-1}{\left|m_{2}\right|} \sim(2 B)^{\alpha-1} .
$$

Therefore, if $\alpha<1, \hat{\mathcal{F}}_{V}^{M}$ decays and 258 is fulfilled.

Since there are $2 B-1$ non-zero masses, and since these masses are either real or appear in complex conjugated pairs, there is at least one mass $m_{l, i}$ such that

$$
t_{i} \equiv \sup _{l \in \mathbb{N}}\left|\tan \arg m_{l, i}\right|<\infty .
$$

Remember that the non-zero masses are confined in the open disc of radius 1 centered at 1 . It follows that $\left|m_{i}\right| \leq\left(1+t_{i}\right) \mathfrak{R} m_{i}$, and thus

$$
\hat{\mathcal{F}}_{V}^{M}>\frac{2}{(2 B)^{2}} \frac{\mathfrak{R} m_{i}}{\left|m_{i}\right|^{2}} \geq \frac{2}{(2 B)^{2}} \frac{1}{\left(1+t_{i}\right)\left|m_{i}\right|} \sim(2 B)^{\alpha-2} .
$$

Therefore, if $\alpha \geq 2, \hat{\mathcal{F}}_{V}^{M}$ does not decay, 258 is violated, and the increasing sequence of quantum graphs is not asymptotically quantum ergodic.

\subsection{The Four Possible Regimes}

To summarise our findings and give a more detailed account of our conjecture and possible obstructions to its validity we have found the following four regimes

1 . The fully universal regime $R_{l} \rightarrow 0$. Equivalently, all matrix elements of the classical map converge to zero, or all eigenvalues apart from the (non-degenerate) eigenvalue one of the classical map $M_{l}$ converge to zero, or all $2 B-1$ non-zero masses converge to one. In this case the eigenvalues of the classical map may be used as a small parameter for a systematic expansion. Our theory then shows that all autocorrelation functions converge to the universal predictions of the Gaussian Random Wave Model, and we believe that the scheme used here can be extended to a rigorous proof. Such graphs are also asymptotically quantum ergodic with a universal rate of convergence.

This class includes the complete DFT graphs (or complete quantum graphs such that nonvanishing elements of the classical map are of order $O\left(V^{-1}\right)$ ). 
2. The large mass regime, characterized by $\alpha<1$ in 260 and $\lim _{l \rightarrow \infty} R_{l} \neq 0$. Some non-zero masses do not converge to one, but none of them approaches zero too fast. The complete Neumann graph is an example in this class with $\alpha=\frac{1}{2}$. Our theory predicts massive corrections to the predictions of the Gaussian Random Wave Model which persist in the asymptotic regime $B \rightarrow \infty$. This implies that the Gaussian Random Wave Model is not applicable but asymptotic quantum ergodicity still holds. This statement has the status of a conjecture which may be very hard to prove because there is no obvious small parameter. As a consequence our result (250) may not estimate the massive contribution accurately. Our numerical data (see Figure 3) indeed show that the intensity correlation matrix for a complete Neumann graph is of a similar order of magnitude as predicted by our theory but its massive contribution is underestimated. More work needs to be done to capture the massive contributions for higher moments correctly. Our theory may be improved considerably by starting from a different exact expression or by going beyond the Gaussian approximation in the massive modes. In the orthogonal case one should start from an expression that incorporates the symmetries of the wave function in all orders.

3. The crossover regime, characterized $1 \leq \alpha \leq 2$ in 260). As $\lim R_{l} \neq 0$ the Gaussian Random Wave Model does not hold. In this regime we conjecture that the criteria (256) or (258) decide whether a sequence of graphs is asymptotically quantum ergodic or not. This conjecture for the crossover regime should be taken with much more care than the previous conjecture for the large mass regime. It does work for Neumann star graphs which have exponent $\alpha=1$ and for which different methods revealed that asymptotic quantum ergodicity does not hold [7]. These graphs have indeed a large number of masses with $m \sim 1 / B$. This number is of order $O(B)$ such that the limit in (258) gives a constant. However, it has also been brought to our attention [41] that analogous criteria derived in [22, 23] for the validity of Gaussian Random Matrix predictions for spectral correlation functions may lead to wrong conclusions for some borderline cases for which the analogous massive contributions are overestimated using the saddle-point approximation to the corresponding exact variant of the supersymmetric $\sigma$-model. To some extent the prediction for the massive correction may be improved as outlined in the large mass regime.

4. The non-universal small mass regime, $\alpha \geq 2$ in 260). We conjecture that neither the Gaussian Random Wave Model nor asymptotic quantum ergodicity hold. In this regime the saddle-point analysis to the exact supersymmetric $\sigma$-model may break down completely. While it may not be trivial to prove this part of our conjecture rigorously our results give very strong evidence in favour of the conjecture.

\section{Discussions}

Our main results are the formula (250) for the autocorrelation functions $C_{[\alpha]}$ defined in (42), and the formula (255) for the fluctuations of an observable defined in (69). 
These formulae depend on the quantum graph only through the matrix $R$, and this matrix, which is defined in (35), only involves the underlying classical dynamics $M$. Hence, our results relate the statistical properties of the quantum energy eigenfunctions to properties of the classical dynamics on the graph. Moreover, they also reveal that the system dependency has no chance to vanish, and hence, a finite graph cannot be entirely described by the Gaussian Random Wave Model developed in Section 3.2 or even be quantum ergodic. These properties can only be met asymptotically in increasing sequences of graphs, that is in sequences of graphs where the number of bonds tends to infinity. In Section 7 classical criteria for such a sequence to be asymptotically described by the Gaussian Random Wave Model or to be asymptotically quantum ergodic are formulated (the section concludes with a summary of the criteria and connected conjectures). The condition $R \rightarrow 0$ for full universality, that is for the Gaussian model to be satisfied in the large graph limit, is more restrictive than the criteria for asymptotic quantum ergodicity. This is understandable since this latter property only depends on the second moment of the intensities, and the fluctuations $\tilde{\mathcal{F}}_{V}$ in (255), which measure the deviation to ergodicity, can also decay in a non-universal way.

The general formulae (250) for the autocorrelation functions and, in particular, (255) for the fluctuations, have been obtained by a saddle-point analysis of the exact field-theoretical expression (148). A comparison with the two periodic orbits approaches in the subsections 3.5 and 4.2 reveals how the field-theoretical scheme exactly proceeds. The first term of $\tilde{\mathcal{F}}_{V}$ in $(255)$, which is universal, originates from our exact calculation on the zero mode manifold, and it coincides with the result predicted by the long diagonal orbits in Subsection 3.5. This draws a parallel between the zero mode, that is the uniform component of the classical map $M$, and long diagonal orbits. This is in fact not surprising since the zero mode is precisely the one that does not decay, and can thus survive in long orbits. The second term of $\tilde{\mathcal{F}}_{V}$ involves the system-dependent matrix $R$, that is the non-zero masses, and it coincides with the system-dependent contribution of the diagonal approximation exposed in Subsection 4.2. Hence, one deduces that our field-theoretical approach discriminates between the different modes of the classical map $M$. The uniform component of $M$ is treated in an exact way, which the diagonal approximation in 4.2 cannot do, while the massive decaying modes are treated in a perturbative way.

It is also interesting to compare our results with those obtained by S. Gnutzmann and A. Altland in [22] and [23] concerning the asymptotic spectral two-point correlation function $R_{2}(s)$ in a sequence of increasing quantum graphs. Their theory relates the function $R_{2}(s)$ to the sequence of spectral gaps $\Delta$ of the matrices $1-M$. If the spectral gaps stay away from zero, the random matrix two-point correlation function is obtained in the limit of large graphs. Our condition $R \rightarrow 0$ for full universality requires all the non-zero eigenvalues of $1-M$ to tend to one, which is obviously much stronger. Hence, even in situations where the Gaussian Random Wave Model does not hold, there is a possibility for random matrix theory to describe $R_{2}(s)$, but if the Gaussian Wave Model does hold, then $R_{2}(s)$ must be universal. Moreover, if the sequence of spectral gaps vanishes as $\Delta_{M} \sim B^{-\alpha}$ as the number of bonds $B$ becomes large, Gnutzmann and Altland's theory predicts different outcomes for $R_{2}(s)$ depending on the value of the positive number $\alpha$. If $\alpha<\frac{1}{2}$, a random matrix behavior is reached, whereas a non-zero system-dependent contribution always remains if $\alpha \geq 1$. In the intermediate regime 
$\alpha \in\left[\frac{1}{2}, 1\right)$, the asymptotic two-point function $R_{2}(s)$ depends on the proportion of vanishing modes, as explained in [23]. In Subsection 7.2] we found that $\alpha<1$ implies asymptotic quantum ergodicity, whereas $\alpha \geq 2$ generally forbids ergodicity. Therefore, universality for $R_{2}(s)$ implies asymptotic quantum ergodicity. However, in the domain $\alpha \in\left[\frac{1}{2}, 1\right)$, quantum ergodicity is always reached, whereas $R_{2}(s)$ can be non-universal.

To conclude, let us mention some possible improvements of our method and some interesting directions for further research. In the main formula 250 , the systemdependent terms correspond to a Gaussian approximation around $Q=\sigma_{3}^{R A}$ in the directions that are transverse to the saddle-point manifold. A true Gaussian approximation should expand the exact action to second order around every point of the saddle-point manifold. The correspondence between these two procedures has only been verified on the submanifold $\tilde{Z}=Z^{\tau}$ with vanishing sources. Moreover and more importantly, in this expansion around the zero mode manifold, the higher order terms have not been controlled. Estimating these terms remains a major problem of this field-theoretical method. Note that only in the fully universal case $R \rightarrow 0$ one knows a small parameter ( $R$ itself) that one may use to order a systematic expansion. Besides, we have also shown that different but equivalent conventions in (76) lead to different outcomes by our second order expansion scheme. This implies that our second order expansion is not a systematic expansion in any intrinsic parameter of the quantum graph. Another question is whether the formula (250) is suitable to describe other quantum systems if the matrix $M$ is replaced with the Perron-Frobenius operator of a chaotic Hamiltonian system. The field-theoretical method used here is probably difficult to generalize to other systems. An idea would be to develop a single periodic orbit approach that reproduces (250) and transfer it to other types of systems.

Acknowledgments This work has been partially supported by ORSAS and the Swiss Society of Friends of the Weizmann Institute of Science. The authors are grateful to Yan Fyodorov, Martin Sieber and Uzy Smilansky for their helpful comments.

\section{References}

[1] A. Bäcker, R. Schubert, P. Stifter, Phys. Rev. E 57, 5425 (1998).

[2] A. Bäcker, R. Schubert, J. Phys. A 35, 527 (2002).

[3] A. Bäcker, R. Schubert, J. Phys. A 35, 539 (2002).

[4] F. Barra, P. Gaspard, J. Stat. Phys. 101, Nos. 1/2 (2000).

[5] F. A. Berezin, Introduction to superanalysis, D. Reidel Publishing Company, Dordrecht, Holland, 1987.

[6] G. Berkolaiko, Proc. Symp. Pure Math. 77, 247 (2009).

[7] G. Berkolaiko, E.B. Bogomolny, J.P. Keating, J. Phys. A 34, 335 (2001).

[8] G. Berkolaiko, J.P. Keating, J. Phys. A 32, 7827 (1999). 
[9] G. Berkolaiko, J.P. Keating, U. Smilansky, Commun. Math. Phys. 273, 137 (2007).

[10] G. Berkolaiko, J.P. Keating, B. Winn, Phys. Rev. Lett. 91, 134103 (2003).

[11] G. Berkolaiko, J.P. Keating, B. Winn, Commun. Math. Phys. 250, 259, (2004).

[12] G. Berkolaiko, B. Winn, arXiv:0801.4104v2, 2008.

[13] M.V. Berry, J. Phys. A 10, 2083 (1977).

[14] M.V. Berry, K.E. Mount, Rep. Prog. Phys. 35, 315 (1972).

[15] J. Bolte and S. Endres, Proc. Symp. Pure Math. 77, 247 (2009).

[16] A. Bouzouina, S. De Bièvre, Commun. Math. Phys. 178, 83 (1996).

[17] Y. Colin de Verdière, Commun. Math. Phys. 102, 497 (1985).

[18] B. Eckhardt, S. Fishman, J.P. Keating, O. Agam, J. Main, K. Müller, Phys. Rev. E 52, 5893 (1995).

[19] K. Efetov, Supersymmetry in disorder and chaos, Cambridge University Press, United Kingdom, 1997.

[20] Y.V. Fyodorov, A.D. Mirlin, IJMPB 8, 3795 (1994).

[21] M. Feingold and A. Peres, Phys. Rev. A 34, 591 (1986).

[22] S. Gnutzmann, A. Atland, Phys. Rev. Lett. 93, 194101 (2004).

[23] S. Gnutzmann, A. Atland, Phys. Rev. E 72, 056215 (2005).

[24] S. Gnutzmann, J.P. Keating, F. Piotet, Phys. Rev. Lett 101, 264102 (2008).

[25] S. Gnutzmann, U. Smilansky, Adv. in Phys. 55, 527 (2006).

[26] S. Gnutzmann, U. Smilansky, J. Weber, Waves in Random Media 14, S61 (2004).

[27] M.C. Gutzwiller, J. Math. Phys 8, 1979 (1967).

[28] R.A. Horn, C.R. Johnson, Matrix Analysis, Cambridge University Press, 1990.

[29] S. Hortikar, M. Srednicki, Phys. Rev. Lett. 80, 1646 (1998).

[30] J.P. Keating, Proc. Symp. Pure Math. 77, 279 (2009).

[31] J.P. Keating, J. Marklof, B. Winn, Commun. Math. Phys. 241, 421, (2003).

[32] J.P. Keating, S.D. Prado, Proc. R. Soc. Lond. A 457, 1855 (2001).

[33] V. Kostrykin, R. Schrader, J. Phys. A 32, 595 (1999).

[34] T. Kottos, U. Smilansky, Phys. Rev. Lett. 79, 4794 (1997). 
[35] T. Kottos, U. Smilansky, Annals of Physics 274, 76 (1999).

[36] A.D. Mirlin, Phys. Rep. 326, 259 (2000).

[37] F. Piotet, Statistical properties of the eigenfunctions on quantum graphs, $\mathrm{PhD}$ thesis, Bristol, 2009.

[38] H. Schanz, T. Kottos, Phys. Rev. Lett. 90, 234101 (2003).

[39] R. Schubert, Ann. Henri Poincaré 7, 1085 (2006).

[40] A.I. Shnirelman, Usp. Mat. Nauk 30, 265 (1975).

[41] I. Smolyarenko, personal communication.

[42] J.D. Urbina, K. Richter, J. Phys. A 36, 495 (2003).

[43] J.D. Urbina, K. Richter, Phys. Rev. Lett. 97, 214101 (2006).

[44] J.D. Urbina, K. Richter, Eur. Phys. J. 145, 255 (2007).

[45] P. Walters, Ergodic theory: Introductory lectures, Lecture Notes in Mathematics 458, Springer-Verlag, Berlin, 1975.

[46] E.P. Wigner, Group theory and its application to quantum mechanics of atomic spectra, Academic Press Inc, New York, 1959.

[47] M. Wilkinson, J. Phys. A 21, 1173 (1988).

[48] S. Zelditch, Commun. Math. Phys. 160, 81 (1994).

[49] S. Zelditch, Commun. in Partial Differential Equations 19, Issue 9 \& 10, 1565 (1994).

[50] S. Zelditch, M. Zworski, Commun. Math. Phys. 175, 673 (1996).

[51] M.R. Zirnbauer, J. Phys. A 29, 7113 (1996). 